UDC: 519.8

\title{
Prediction of moving and unexpected motionless bottlenecks based on three-phase traffic theory
}

\author{
S. L. Klenov ${ }^{1, a}$, D. Wegerle ${ }^{2, b}$, B. S. Kerner ${ }^{2, c}$, M. Schreckenberg ${ }^{2, d}$ \\ ${ }^{1}$ Moscow Institute of Physics and Technology, Department of Physics, \\ Institutskiy per. 9, Dolgoprudny, Moscow Region, 141700, Russia \\ ${ }^{2}$ Physics of Transport and Traffic, University Duisburg-Essen, \\ Forsthausweg 2, 47048 Duisburg, Germany \\ E-mail: ${ }^{a}$ klenov.sl@mipt.ru, ${ }^{b}$ dominik.wegerle@uni-due.de, ${ }^{\mathrm{c}}$ boris.kerner@uni-due.de, \\ d michael.schreckenberg@uni-due.de
}

Received 28.01.2021, after completion - 17.02.2021.

Accepted for publication 19.02.2021.

\begin{abstract}
We present a simulation methodology for the prediction of "unexpected" bottlenecks, i.e., the bottlenecks that occur suddenly and unexpectedly for drivers on a highway. Such unexpected bottlenecks can be either a moving bottleneck (MB) caused by a slow moving vehicle or a motionless bottleneck caused by a stopped vehicle (SV). Based on simulations of a stochastic microscopic traffic flow model in the framework of Kerner's three-phase traffic theory, we show that through the use of a small share of probe vehicles (FCD) randomly distributed in traffic flow the reliable prediction of "unexpected" bottlenecks is possible. We have found that the time dependence of the probability of MB and SV prediction as well as the accuracy of the estimation of MB and SV location depend considerably on sequences of phase transitions from free flow (F) to synchronized flow ( $\mathrm{S}$ ) ( $\mathrm{F} \rightarrow \mathrm{S}$ transition) and back from synchronized flow to free flow ( $\mathrm{S} \rightarrow \mathrm{F}$ transition) as well as on speed oscillations in synchronized flow at the bottleneck. In the simulation approach, the identification of $\mathrm{F} \rightarrow \mathrm{S}$ and $\mathrm{S} \rightarrow \mathrm{F}$ transitions at an unexpected bottleneck has been made in accordance with Kerner's three-phase traffic theory. The presented simulation methodology allows us both the prediction of the unexpected bottleneck that suddenly occurs on a highway and the distinguishing of the origin of the unexpected bottleneck, i.e., whether the unexpected bottleneck has occurred due to a MB or a SV.
\end{abstract}

Keywords: traffic simulation, traffic breakdown, moving bottleneck, Kerner's three-phase traffic theory, microscopic stochastic three-phase traffic model, prove vehicles (floating car data - FCD)

Citation: Computer Research and Modeling, 2021, vol. 13, no. 2, pp. 319-363 (Russian).

The authors thank the partners for their support within the project MEC-View - Mobile Edge Computing basierte Objekterkennung für hoch- und vollautomatisiertes Fahren, funded by the German Federal Ministry of Economics and Energy by resolution of the German Federal Parliament.

(c) 2021 Sergey L. Klenov, Dominik Wegerle, Boris S. Kerner, Michael Schreckenberg This work is licensed under the Creative Commons Attribution-NoDerivs 3.0 Unported License. To view a copy of this license, visit http://creativecommons.org/licenses/by-nd/3.0/ or send a letter to Creative Commons, PO Box 1866, Mountain View, CA 94042, USA. 
УДК: 519.8

\title{
Обнаружение медленно движущихся или неожиданно возникающих неподвижных «бутылочных горлышек» в транспортном потоке на основе теории трех фаз
}

\author{
С. Л. Кленов ${ }^{1, \mathrm{a}}$, Д. Вегерле $\mathrm{e}^{2, \mathrm{~b}}$, Б. С. Кернер ${ }^{2, \mathrm{c}}$, М. Шрекенберг ${ }^{2, \mathrm{~d}}$ \\ ${ }^{1}$ Московский физико-технический институт (национальный исследовательский университет), \\ кафедра общей физики, \\ Россия, 141700, Московская область, г. Долгопрудный, Институтский пер., д. 9 \\ ${ }^{2}$ Университет Дюсбург-Эссен, физика транспорта и траффика, \\ Германия, 47048, г. Дюсбург, Форстхаусвег 2 \\ E-mail: ${ }^{a}$ klenov.s1@mipt.ru, ${ }^{\mathrm{b}}$ dominik.wegerle@uni-due.de, ${ }^{\mathrm{c}}$ boris.kerner@uni-due.de, \\ d michael.schreckenberg@uni-due.de
}

Получено 28.01.2021, после доработки - 17.02.2021.

Принято к публикации 19.02.2021.

\begin{abstract}
Разработан метод обнаружения неожиданно возникающих «бутылочных горлышек», которые появляются в транспортном потоке внезапно и неожиданно для водителей. Такие неожиданно возникающие бутылочные горлышки могут двигаться, если они вызваны медленно движущейся автомашиной (тип MB), или же оставаться неподвижными, если они вызваны внезапно остановившейся автомашиной (тип SV), например, в результате аварии. На основе численного моделирования стохастической микроскопической модели транспортного потока в рамках теории трех фаз Кернера показано, что даже при использовании небольшого процента «зондирующих» (измеряющих) автомашин (FCD), случайным образом распределенных в транспортном потоке, возможно надежное обнаружение неожиданно возникающих бутылочных горлышек. Найдено, что временная зависимость вероятности прогноза бутылочных горлышек типа МВ или SV, а также точность определения их положения существенно зависят от последовательности фазовых переходов от свободного $(\mathrm{F})$ к синхронизованному $(\mathrm{S})$ транспортному потоку $(\mathrm{F} \rightarrow \mathrm{S}$-переход) и обратных фазовых переходов $(\mathrm{S} \rightarrow \mathrm{F}$-переход), а также от колебаний скорости автомашин в синхронизованном потоке вблизи бутылочного горлышка. Предлагаемая численная методика позволяет как обнаруживать неожиданно возникшее бутылочное горлышко на автомагистрали, так и различать, связано ли такое бутылочное горлышко с медленно движущейся автомашиной (MB) или же с внезапно остановившейся автомашиной (SV).
\end{abstract}

Ключевые слова: моделирование транспортных потоков, переход к плотному потоку, движущееся бутылочное горлышко, теория трех фаз Кернера, зондирующие автомашины (FCD) и навигационные данные

Авторы благодарят партнеров за их поддержку в рамках проекта MEC-View - Mobile Edge Computing basierte Objekterkennung für hoch- und vollautomatisiertes Fahren, финансируемого Федеральным министерством Германии по экономике и энергии с разрешения Федерального Парламента Германии.

(c) 2021 Сергей Львович Кленов, Доминик Вегерле, Борис Семенович Кернер, Михаель Шрекенберг Статья доступна по лицензии Creative Commons Attribution-NoDerivs 3.0 Unported License. Чтобы получить текст лицензии, посетите веб-сайт http://creativecommons.org/licenses/by-nd/3.0/ или отправьте письмо в Creative Commons, PO Box 1866, Mountain View, CA 94042, USA. 


\section{Introduction}

To increase traffic safety in mixed traffic flow consisting of automated driving and human driving vehicles, automated driving vehicles should be provided with reliable information about traffic congestion. Congested traffic occurs due to traffic breakdown at road bottlenecks like on-ramp and off-ramp bottlenecks, etc. Additionally, a slow vehicle moving on a highway with a random number of lanes and even when the slow vehicle is not forced to move in a specific lane can cause a moving bottleneck (MB) [Gazis, Herman, 1990; Newell, 1993; Newell, 1998]. Newell [Newell, 1993; Newell, 1998] revealed that in a system coordinate moving at MB speed traffic breakdown at the MB should exhibit qualitatively the same features as those at road bottlenecks. Road bottlenecks can be known from a digital map. Therefore, the recognition of road bottlenecks can be made automatically inside a vehicle. There are numerous papers in which a variety of approaches to the recognition of road bottlenecks have been developed (e.g., [Yue et al., 2018; Zheng et al., 2011; Ke et al., 2018; Zhang et al., 2018]).

In contrast to road bottlenecks, an MB can appear unexpectedly, for example, due to the merging of a slow vehicle from an on-ramp. There is also another reason for the unexpected occurrence of a bottleneck: an unexpected stop of one of the vehicles. Such a stopped vehicle (SV) causes the occurrence of the motionless bottleneck on the road (called SV bottleneck for short). A probable reason for the bottleneck caused by a stopped vehicle is a broken-down vehicle in real traffic. Prediction of an MB has already been studied in [Wegerle et al., 2020]. However, when a bottleneck occurs unexpectedly on a highway, we do not know initially whether the bottleneck is caused by an MB or an SV. The methodology of [Wegerle et al., 2020], which has been developed for an MB only, cannot be used for SV bottleneck prediction. In this paper, we present a general methodology for the prediction of an unexpected bottleneck. This general methodology based on Kerner's three-phase traffic theory allows us both to predict an unexpected bottleneck that suddenly occurs on a highway and to distinguish of the origin of the unexpected bottleneck, i.e., whether the unexpected bottleneck has occurred due to an MB or an SV.

Usually, simulations of traffic breakdown are made with classical (standard) traffic flow models (e.g., [Gazis, 2006; Elefteriadou, 2014; Helbing, 2001]). Standard traffic flow models have also been used for simulations of an MB [Munoz, Daganzo, 2002; Daganzo, Laval, 2003; Daganzo, Laval, 2005a; Gazis, Herman, 1990; Lebacque et al., 1998; Leclercq et al., 2004; Fadhloun et al., 2014a; Fadhloun et al., 2014b; Newell, 1993; Newell, 1998].

However, real field traffic data shows (see [Kerner, 2004]) that traffic breakdown at a bottleneck is a phase transition from free flow to synchronized flow ( $\mathrm{F} \rightarrow \mathrm{S}$ transition) that exhibits an empirical nucleation nature. As explained in detail in [Kerner, 2009; Kerner, 2018a; Kerner, 2019], none of the standard traffic flow models can show and explain the empirical nucleation nature of traffic breakdown $(\mathrm{F} \rightarrow \mathrm{S}$ transition). For this reason, all simulations in this paper will be made with a microscopic traffic flow model in the framework of the three-phase traffic theory [Kerner, Klenov, 2010] that explains the empirical nucleation nature of traffic breakdown.

\section{Objective of the Paper}

The objective of this paper is as follows: We present a general methodology how to predict traffic congestion either at an MB or at a motionless bottleneck caused by SV with the use of probe vehicles (FCD). Additionally, we study traffic phenomena that reduce the reliability of MB or SV prediction. This general methodology is illustrated with simulations of MB and SV scenarios. A statistical analysis of the quality of $\mathrm{MB}$ and $\mathrm{SV}$ prediction is made.

Because the scenarios of MB and SV are similar to each other, to explain the objective of the paper, we discuss the MB scenario. We consider a highway in which an MB appears suddenly. This can 
be realized, for example, when a slowly moving vehicle has merged from an on-ramp onto the main road. Because the slow vehicle moves in the flow direction, the MB location is moving alongside. We assume that due to the existence of the $\mathrm{MB}$ traffic breakdown ( $\mathrm{F} \rightarrow \mathrm{S}$ transition) can occur spontaneously at the MB. As a result of traffic breakdown, synchronized flow occurs at the MB.

While the MB moves in traffic flow, the region of synchronized flow at the MB is also moving. Thus, vehicles approaching the MB must decelerate to synchronized flow at the MB. We assume in the paper that traffic flow downstream of the MB is free flow. For this reason, after vehicles have passed the $\mathrm{MB}$, vehicles can accelerate from synchronized flow at the MB to free flow downstream of the MB. Therefore, while passing the MB, vehicles accelerate from synchronized flow at the MB to free flow downstream of the MB. We designate the transition from synchronized flow (S) to free flow $(F)$ on a trajectory of a vehicle as an $S_{F}$ phase transition point. $S_{F}$ phase transition points will be explained in more detail below where it will be shown that they are located in the MB vicinity.

We assume that there are probe vehicles (FCD) that are randomly distributed between other vehicles in traffic flow. A probe vehicle produces a chain of GPS vehicle locations. The probe vehicle is connected with a data center. The probe vehicle sends the chain automatically to the data center. We assume also that an analysis of the GPS-location chains received from probe vehicles makes it possible to recognize $\mathrm{S}_{\mathrm{F}}$ phase transition points on trajectories of the probe vehicles (see, e.g., [Kerner et al., 2013]).

In the data center ${ }^{1}$, the information about $S_{\mathrm{F}}$ phase transition points is used for a decision whether or not there is an MB in traffic flow. In particular, the $S_{\mathrm{F}}$ phase transition points are used to find the approximation of the location and the speed of a single front boundary of the MB. To explain these results, traffic dynamics at the MB and the SV are studied in the framework of Kerner's three-phase traffic theory. A general methodology of the procedure for the prediction of either an MB or an SV, which is the main objective of this paper, will be considered below.

As we will show below, there can be large errors in the definition of the location of an MB or $\mathrm{SV}$ as well as large errors in the definition of the MB speed. The large errors are caused by random fluctuations in locations of $\mathrm{S}_{\mathrm{F}}$ phase transition points relative to the real MB or SV location. For this reason, to recognize an $\mathrm{MB}$ or $\mathrm{SV}$, the data center needs a sequence of several $\mathrm{S}_{\mathrm{F}}$ phase transition points from different probe vehicles. Therefore, the data center can recognize either the MB or the SV bottleneck through the use of the probe vehicles with some probability only. The larger the number of $\mathrm{S}_{\mathrm{F}}$ phase transition points, the larger is the probability of the recognition of either an MB or an SV. Thus, the probability of the recognition of either an MB or an SV should be an increasing function of both the rate of probe vehicles $\gamma$ and the time. For this reason, one of the important objectives of this paper is to study the probability of the prediction of either an MB or an SV as a time function for different values of $\gamma$.

It should be noted that a probe vehicle can be a human controlled vehicle without an automated vehicle system. In other words, probe vehicles are connected vehicles that can include both automated driving and human driving vehicles. For this reason, the rate of automated driving vehicles in traffic flow can be considerably smaller than the rate of probe vehicles $\gamma$. The methodology of the prediction of either an MB or an SV developed in the paper can be used for ITS-applications even in the absence of automated driving vehicles in traffic flow. The peculiarity of an automated driving vehicle is that the automated system in the vehicle can change its behavior automatically based on the prediction of either an MB or an SV.

After either an MB or an SV has been recognized, the data center can send the information about the MB speed (if the unexpected bottleneck is the MB) and time-function of the location of either an $\mathrm{MB}$ or an SV to automated driving vehicles. This information can be used by either automated

\footnotetext{
${ }^{1}$ Methods of traffic data measurements have been reviewed in the book by Rehborn et al. [Rehborn et al., 2020]. In this book readers can also find a history of the emergence of Kerner's three phase traffic theory.
} 
driving vehicles or other ITS-applications for speed harmonization, collision avoidance that should increase traffic safety and comfort. It should be noted that probe vehicles can also be connected with some other connected vehicles, including automated driving vehicles. In this case, automated driving vehicles have the possibility of receiving the same information from probe vehicles as that received by the data center. Therefore, automated driving vehicles can use the methodology of MB prediction developed in this paper inside the automated driving vehicles without the use of data center information. However, a more detailed consideration of these and other possible ITS-applications of the prediction of either an MB or an SV presented in the paper is beyond the scope of this paper.

It should be emphasized that there are other qualitatively different approaches associated with the prediction of the location of road bottlenecks (for example, based on wavelet transform methods) (see, e.g., [Yue et al., 2018; Zheng et al., 2011; Ke et al., 2018; Zhang et al., 2018]). However, we do not know any publications in the literature in which the methodology of the prediction of either an MB or an SV and the distinguishing between these unexpected bottlenecks developed in this paper has been presented before.

Moreover, we should mention that when either an $\mathrm{MB}$ or an SV is simulated with standard traffic flow models [Munoz, Daganzo, 2002; Daganzo, Laval, 2003; Daganzo, Laval, 2005a; Gazis, Herman, 1990; Lebacque et al., 1998; Leclercq et al., 2004; Fadhloun et al., 2014a; Fadhloun et al., 2014b; Newell, 1993; Newell, 1998], the models cannot show the empirical nucleation nature of traffic breakdown [Kerner, 2009; Kerner, 2018a; Kerner, 2019]. The nucleation nature of traffic breakdown at a bottleneck is the basic feature of Kerner's three-phase traffic theory that has been implemented in the Kerner-Klenov stochastic microscopic model (KK model for short) used for all simulations presented in this paper.

\section{Background: Simulation Approach based on Kerner's Three-Phase Traffic Theory}

\section{Three-Phase Traffic Theory}

Three-phase traffic theory introduced by Kerner (see [Kerner, 2004]) is the framework for the understanding of empirical states of traffic flow in three phases: (i) free flow (F), (ii) synchronized flow (S), (iii) wide moving jam (J). The synchronized flow and wide moving jam phases belong to congested traffic. A wide moving jam (J) exhibits the characteristic feature to propagate through a bottleneck while maintaining the mean velocity of the downstream jam front. In contrast, the downstream front of synchronized flow phase does not maintain the mean velocity of the downstream front; in particular, the downstream front of synchronized flow (S) is usually fixed at a bottleneck.

\section{Main Reason for Three-Phase Traffic Theory}

The main reason for three-phase traffic theory is the explanation of the empirical nucleation nature of traffic breakdown ( $\mathrm{F} \rightarrow \mathrm{S}$ transition) at a highway bottleneck.

\section{Empirical Nucleation Nature of Traffic Breakdown at Highway Bottlenecks}

Empirical data shows that traffic breakdown at a highway bottleneck is a phase transition from free flow to synchronized flow ( $\mathrm{F} \rightarrow \mathrm{S}$ transition). Empirical traffic breakdown ( $\mathrm{F} \rightarrow \mathrm{S}$ transition) can be induced at the bottleneck (see, e.g., Fig. 2.16 in [Kerner, 2004]). In contrast to the effect of "spillover" at which congestion at the bottleneck exists due to the existence of downstream congestion, after an induced $\mathrm{F} \rightarrow \mathrm{S}$ transition has occurred at a bottleneck, free flow is downstream of the bottleneck: there is no downstream congestion any more. The empirical induced traffic breakdown means that the $\mathrm{F} \rightarrow \mathrm{S}$ transition occurs in a metastable state of free flow with respect to the $\mathrm{F} \rightarrow \mathrm{S}$ transition: Small 
speed disturbances in metastable free flow decay. However, a large enough local speed disturbance in metastable free flow (e.g., a localized congested pattern approaching the bottleneck shown in Fig. 2.16 in [Kerner, 2004]) does lead to the $\mathrm{F} \rightarrow \mathrm{S}$ transition. A local speed disturbance that leads to the $\mathrm{F} \rightarrow \mathrm{S}$ transition is called a nucleus for traffic breakdown. A detailed consideration of the empirical nucleation nature of traffic breakdown can be found in Chap. 3 of [Kerner, 2017].

\section{Main Prediction of Three-Phase Traffic Theory}

The main prediction of three-phase flow theory is that free flow at the bottleneck can be in a metastable state with respect to an $\mathrm{F} \rightarrow \mathrm{S}$ transition. In its turn, this nucleation nature of the $\mathrm{F} \rightarrow \mathrm{S}$ transition is governed by the nucleation nature of an $\mathrm{S} \rightarrow \mathrm{F}$ instability in synchronized flow (see [Kerner, 2015]). A detailed consideration of Kerner's three-phase traffic theory can be found in the books [Kerner, 2004; Kerner, 2009; Kerner, 2017].

\section{Classical Models and Stochastic Microscopic Three-Phase Traffic Flow Model}

The metastability of free flow with respect to the $\mathrm{F} \rightarrow \mathrm{S}$ transition at the bottleneck can be shown by none of the standard traffic models used and/or reviewed, for example, in [Gazis, 2006; Elefteriadou, 2014; Helbing, 2001; Munoz, Daganzo, 2002; Daganzo, Laval, 2003; Daganzo, Laval, 2005a; Gazis, Herman, 1990; Lebacque et al., 1998; Leclercq et al., 2004; Fadhloun et al., 2014a; Fadhloun et al., 2014b; Newell, 1993; Newell, 1998]. This statement has been proven in detail in Chap. 10 of [Kerner, 2009] and in [Kerner, 2018a]. Therefore, we use the microscopic stochastic threephase traffic flow KK model [Kerner, Klenov, 2010] that mathematical formulation has been reviewed in [Kerner, 2017, Appendix A (Pages 553-623)]. To explain the model, we consider a 2Z-characteristic for phase transitions simulated with the model (Fig. 1) [Kerner, Klenov, 2010].

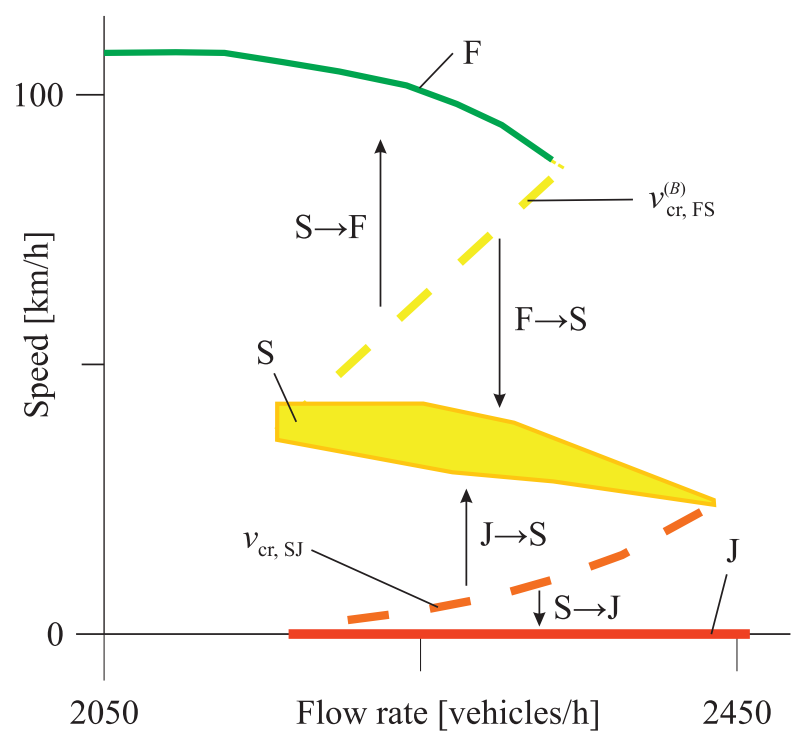

Figure 1. Simulated 2Z-characteristic for phase transitions in the speed-flow rate plane. Traffic breakdown is described by the first Z-characteristic between free flow (F) and synchronized flow (S) (2D-region). An F $\rightarrow S$ transition (traffic breakdown) at the bottleneck can occur only if a nucleus appears at the bottleneck. The nucleus is a local speed disturbance whose minimum speed is equal to or smaller than a critical value $v_{\mathrm{cr} \text {, FS }}^{\text {(d) }}$ (dashed yellow curve). The second Z-characteristic is related to a phase transition between synchronized flow (S) and wide moving jam $(\mathrm{J})(\mathrm{S} \rightarrow \mathrm{J}$ transition). The wide moving jam occurs when the minimum speed within a moving local speed decrease in synchronized flow is smaller than a critical speed $v_{\mathrm{cr}}$, SJ (dashed red curve). Adapted from [Kerner, 2017] 
Traffic breakdown is described in the model by the first Z-characteristic between free flow (F) and synchronized flow (S) (2D-region) in the speed-flow rate plane. An $\mathrm{F} \rightarrow \mathrm{S}$ transition (traffic breakdown) at the bottleneck can occur only if a nucleus appears at the bottleneck. The nucleus is a local speed disturbance whose minimum speed is equal to or smaller than a critical value $v_{\mathrm{cr} \text {, FS }}^{(\mathrm{B})}$ (dashed curve in Fig. 1). In this case, traffic breakdown occurs. Otherwise, when the minimum speed within the local speed disturbance is larger than $v_{\text {cr, FS }}^{(\mathrm{B})}$, due to the $\mathrm{S} \rightarrow \mathrm{F}$ instability, the disturbance decays; therefore, no traffic breakdown occurs. The $\mathrm{S} \rightarrow \mathrm{F}$ instability is a growing wave of a local speed increase in synchronized flow (detailed explanations of the $\mathrm{S} \rightarrow \mathrm{F}$ instability can be found in [Kerner, 2017]). The Z-characteristic between the phases F and S cannot be simulated with the standard models; therefore, we do not use them. The second Z-characteristic between synchronized flow (S) and wide moving jam (J) (Fig. 1) can be simulated by most standard traffic flow models in which classical traffic flow instability introduced in 1950s is used (see references in [Gazis, 2006; Helbing, 2001]). In contrast to the $\mathrm{S} \rightarrow \mathrm{F}$ instability of the three-phase theory, the classical traffic flow instability is a growing wave of a local speed reduction in synchronized flow that occurs when the minimum speed within a local speed decrease in synchronized flow is smaller than a critical speed $v_{\text {cr, SJ }}$ and it can lead to wide moving jam emergence [Kerner, 2004]. However, wide moving jams do not occur in simulations presented below; therefore, they will not be discussed any more.

\section{About Empirical Validation and Calibration of Kerner-Klenov (KK) Stochastic Microscopic Model}

The stochastic microscopic KK model has been used in all simulations made in this paper. However, the question can arise whether reliable validations and parameter calibration of this model with the use of real field data have been made.

Traffic occurs in space and time. Traffic breakdown leading to traffic congestion occurs usually at bottlenecks. As emphasized above, the empirical nucleation nature of traffic breakdown $(\mathrm{F} \rightarrow \mathrm{S}$ transition) at the bottleneck is the reason for Kerner's three-phase traffic theory. The theory is a qualitative explanation of real field traffic data [Kerner, 1998a; Kerner, 1998b; Kerner, 1999a; Kerner, 1999b]. The first mathematical model that was able to simulate the empirical nucleation nature of traffic breakdown $(\mathrm{F} \rightarrow \mathrm{S}$ transition) at the bottleneck was the KK stochastic microscopic model used for all simulations in this paper [Kerner, Klenov, 2002; Kerner, Klenov, 2003]. In 2003, the model was validated and calibrated based on a huge number of spatiotemporal traffic data measured at different highways during 1996-2001. Later, special empirical validations of the model were made [Kerner et al., 2006; Kerner et al., 2007] based on real field data measured during 2002-2006. In this empirical test of the model, it was found that this model can be used for a reliable numerical study of the performance of a variety of ITS-applications (see references in [Kerner, 2004; Kerner, 2009; Kerner, 2017; Kerner, 2018a; Kerner, 2019]). In 2013, the KK stochastic microscopic model was calibrated based on TomTom empirical probe vehicle data [Kerner et al., 2013]. It was found that no change of model parameters was needed to show quantitative spatiotemporal characteristics of traffic patterns found in TomTom probe vehicle data. With the use of the KK model, in [Kerner, 2015] sequences of $\mathrm{F} \rightarrow \mathrm{S} \rightarrow \mathrm{F}$ transitions before traffic breakdown at bottlenecks were predicted. The sequences were indeed observed in recent empirical studies of probe vehicle data [Molzahn et al., 2017; Duelgar et al., 2019].

\section{Methodology of Prediction of a Moving Bottleneck}

\section{Prediction of a Moving Bottleneck: Simulations of On-Line Application}

A methodology of MB prediction that can be used for on-line applications is as follows:

1. We assume a random distribution of FCD-vehicles (probe vehicles) with rate $\gamma$ between other vehicles, which in simulations is called a random realization (or a sample) of FCD-vehicles. 
2. Based on results of [Kerner et al., 2013], through the use of FCD-vehicles in the realization for each time instant we prove whether $S_{F}$ phase transition points (from the phase $S$ to the phase $F$ ) have been registered; the number of the $\mathrm{S}_{\mathrm{F}}$ points is denoted by $K$.

3. If through the use of FCD-vehicles a $S_{F}$ phase transition point has been registered in the realization, we can assume that there is a bottleneck in traffic flow. Through the use of a digital map, we prove whether the location of the $S_{F}$ phase transition point is correlated with one of the locations of potential road bottlenecks. In this paper, we assume that there is no such correlation. Without some additional analysis we do not know the type of the bottleneck related to the $\mathrm{S}_{\mathrm{F}}$ phase transition point.

4. A bottleneck could be identified as an MB only if at some time instant $t$ there are at least two $\mathrm{S}_{\mathrm{F}}$ phase transition points:

$$
K(t) \geq 2
$$

5. Under condition (1), to estimate the speed of the bottleneck, we apply a standard linear regression through the $S_{F}$ phase transition points in the realization [Draper, Smith, 1998]:

$$
v_{\mathrm{rec}}(K)=\frac{\sum_{i=1}^{K}\left(t_{i}-\bar{t}\right) \cdot\left(x_{i}-\bar{x}\right)}{\sum_{i=1}^{K}\left(t_{i}-\bar{t}\right)^{2}} \text { at } K \geq 2 .
$$

The bottleneck location denoted by $x_{\text {rec }}$ can be estimated as a time function:

$$
x_{\mathrm{rec}}(t)=v_{\mathrm{rec}}(K(t))(t-\bar{t}(K(t)))+\bar{x}(K(t)) .
$$

6. The standard error $\sigma_{\text {rec }}(K)$ for the estimated bottleneck speed $v_{\text {rec }}(K)$ is found from the formula

$$
\sigma_{\mathrm{rec}}(K)=\sqrt{\frac{\sum_{i=1}^{K}\left(x_{i}-x_{\mathrm{rec}}\left(t_{i}\right)\right)^{2}}{(K-2) \sum_{i=1}^{K}\left(t_{i}-\bar{t}\right)^{2}}} \text { at } K>2,
$$

where $v_{\text {rec }}(K)$ is the bottleneck speed related to the realization in which a sequence of the $\mathrm{S}_{\mathrm{F}}$ phase transition points is registered through the use of FCD-vehicles; $t_{i}$ and $x_{i}, i=1,2, \ldots, K$ are, respectively, the time instant and location at which a $S_{F}$ phase transition point has been registered; $K$ is the number of the $\mathrm{S}_{\mathrm{F}}$ phase transition points (the number of points $\mathrm{S}_{\mathrm{F}}$ related to $\left.\left[t_{i}, x_{i}\right] \forall t_{i} \leq t\right)$ registered through the use of FCD-vehicles in the realization by time instant $t$, therefore, $K$ increases with time: $K=K(t) ; \bar{t}(K)=(1 / K) \sum_{i=1}^{K} t_{i} ; \bar{x}(K)=(1 / K) \sum_{i=1}^{K} x_{i}$.

7. As we will show below, $S_{\mathrm{F}}$ phase transition points registered through the use of FCD-vehicles are located in the bottleneck vicinity. This allows us to draw the following conclusions: Using the estimated bottleneck speed $v_{\text {rec }}(2)$ and standard error $\sigma_{\text {rec }}(4)$, at a given confidence level $p_{\alpha}$ (where $1-p_{\alpha} \ll 1$ ), the bottleneck type and bottleneck speed can be estimated, if the following conditions (5) and (6) are calculated simultaneously:

$$
\begin{gathered}
v_{\mathrm{rec}}(K)-t_{\alpha, K-2}^{(1)} \sigma_{\mathrm{rec}}(K)>0, \\
v_{\mathrm{rec}}(K)-t_{\alpha, K-2}^{(2)} \sigma_{\mathrm{rec}}(K) \geq-\Delta_{\mathrm{SV}}, \\
v_{\mathrm{rec}}(K)+t_{\alpha, K-2}^{(2)} \sigma_{\mathrm{rec}}(K) \leq \Delta_{\mathrm{SV}},
\end{gathered}
$$


where $\Delta_{\mathrm{SV}}$ is a given small threshold speed of the bottleneck $\left(\Delta_{\mathrm{SV}}>0\right.$; for example, the value $\Delta_{\mathrm{SV}}$ can be chosen as $1-2 \mathrm{~km} / \mathrm{h}$ ); values $t_{\alpha, K-2}^{(1)}$ and $t_{\alpha, K-2}^{(2)}$ are found from well-known Student's t-distribution tables [Draper, Smith, 1998], respectively, within one-sided and twosided regions with $K-2$ degrees of freedom for the given confidence level $p_{\alpha}$. We denote $K_{\mathrm{MB}}$ as the value $K=K\left(t_{\mathrm{MB}}\right)$ at which condition (5) is satisfied for any time $t \geq t_{\mathrm{MB}}$; respectively, $K_{\mathrm{SV}}$ is the value $K=K\left(t_{\mathrm{SV}}\right)$ at which condition (6) is satisfied for any time $t \geq t_{\mathrm{SV}}$.

8. If condition (5) is satisfied, i.e., $K=K_{\mathrm{MB}}$, we can state that at the given confidence level $p_{\alpha}$ the bottleneck is an MB.

9. If condition (6) is satisfied, i.e., $K=K_{\mathrm{SV}}$, the bottleneck speed is smaller than the threshold $\Delta_{\mathrm{SV}}$. Thus, at the given confidence level $p_{\alpha}$ the bottleneck can be either an MB that moves at a very small speed $0<v_{\text {rec }}<\Delta_{\mathrm{SV}}$ or an SV bottleneck caused by a stopped vehicle (SV) at $v_{\mathrm{MB}}=0$.

10. The MB speed (2) as well as the bottleneck location (3) are sent to automated driving vehicles.

\section{Probability of Prediction of Bottleneck: Statistical Analysis}

The reliability of this methodology of on-line bottleneck prediction can be found from the following statistical off-line data analysis:

- We repeat the above methodology for each of a large number $M$ (where $M \gg 1$ ) different random realizations.

- We calculate the total number of realizations $m_{\mathrm{MB}}(t)$ and $m_{\mathrm{SV}}(t)$ (where $m_{\mathrm{MB}}(t) \leq M$ and $\left.m_{\mathrm{SV}}(t) \leq M\right)$ in which at time instant $t$ either an MB or an SV bottleneck has been identified at the confidence level $p_{\alpha}$.

- Probability $P_{\mathrm{MB}}(t)$ or probability $P_{\mathrm{SV}}(t)$ that at time instant $t$ at the confidence level $p_{\alpha}$ either an MB or an SV bottleneck is identified in traffic flow are

$$
\begin{aligned}
P_{\mathrm{MB}}(t) & =\frac{m_{\mathrm{MB}}(t)}{M}, \\
P_{\mathrm{SV}}(t) & =\frac{m_{\mathrm{SV}}(t)}{M} .
\end{aligned}
$$

- We repeat this calculation of either $P_{\mathrm{MB}}(t)$ or $P_{\mathrm{SV}}(t)$ for different rates $\gamma$ of probe vehicles.

- We study errors in the estimated bottleneck location. We calculate the mean bottleneck location $\bar{x}_{\text {rec }}(t)$ averaged over all $M$ realizations:

$$
\bar{x}_{\mathrm{rec}}(t)=\frac{1}{R(t)} \sum_{r=1}^{R(t)} x_{\mathrm{rec}}^{(\mathrm{r})}(t),
$$

where $x_{\mathrm{rec}}^{(\mathrm{r})}(t)$ is the estimated bottleneck location at time instant $t$ for realization $r, r=1, \ldots, R$; $R=R(t)$ is the number of realizations for which at time instant $t$ the estimation of the MB location is possible, $1 \leq R \leq M$.

- We identify those traffic phenomena at the bottleneck that reduce the probabilities $P_{\mathrm{MB}}(t)$ and $P_{\mathrm{SV}}(t)$. 


\section{Application of Methodology of Prediction of a Moving Bottleneck for Simulations of a Moving Bottleneck Scenario}

To illustrate the above general methodology, we consider a special MB scenario in which an MB is caused by a slow vehicle moving in the right lane on the two-lane road. This scenario is the usual one for Germany where slow vehicles should usually move in the right lane on two-lane highways. Simulations made with the KK model have been done under open boundary conditions; in the model discrete time $t=n \tau$ is used, where $n=0,1,2, \ldots ; \tau=1 \mathrm{~s}$ is a time step. Simulation parameters are chosen according to [Kerner, Klenov, 2010, Figure 6(c)]: The MB speed $v_{\mathrm{MB}}=28.8 \mathrm{~km} / \mathrm{h}$ and the flow rate per lane at the beginning of the road is equal to $q_{\text {in }}=1375$ vehicles/(h lane). The choice of a small and time-independent speed of the $\mathrm{MB} v_{\mathrm{MB}}=28.8 \mathrm{~km} / \mathrm{h}$ can be explained as follows. We assume that a heavy slow vehicle (for example, required for a road construction and repair objectives) can be considered a danger for automated driving vehicles. Often such a heavy vehicle has the maximum speed of about (or even less than) $30 \mathrm{~km} / \mathrm{h}$. We assume also that downstream of such a heavy slow vehicle free flow is realized and there are no road gradients that can effect the maximum speed of the heavy vehicle. The heavy slow vehicle that moves at its maximum speed is the reason for the MB. Thus, the speed of the MB $v_{\mathrm{MB}}$ is equal to the maximum speed of the heavy vehicle.

Behind the MB we assume an area with a length of $300 \mathrm{~m}$, where vehicles moving in the right lane try to change the lane by specific lane changing rules [Kerner, Klenov, 2010, Appendix A.2]. The simulation covers a period of 42 minutes and a road length of 20 kilometers. Simulations of traffic breakdown at the MB show that at the chosen flow rate and MB speed the speed of other vehicles is effected by the MB only in an MB vicinity, while undisturbed free flow is realized far enough upstream of the MB and about $0.5 \mathrm{~km}$ downstream of the MB (Fig. 2).

As is well known [Kerner, Klenov, 2010], traffic breakdown at the MB is a random timedelayed $\mathrm{F} \rightarrow \mathrm{S}$ transition occurring in metastable free flow. This means that for traffic breakdown (arrow "F $\rightarrow \mathrm{S}$ transition" in Fig. 2) a large enough local speed disturbance (nucleus for traffic breakdown) should randomly appear in free flow at the MB. Because the occurrence of such a large amplitude disturbance is a random effect, traffic breakdown occurs after a random time delay denoted by $\mathrm{T}^{(\mathrm{B})}$ in Fig. 2. As shown by [Kerner, Klenov, 2010] and explained by [Kerner, 2004; Kerner, 2017], all probabilistic features of a $\mathrm{F} \rightarrow \mathrm{S}$ transition at the $\mathrm{MB}$ are qualitatively the same for any other road bottleneck. In particular, if we transform speed data shown in Fig. 2 in the motion-less coordinate system into a coordinate system moving at the MB speed $v_{\mathrm{MB}}=28.8 \mathrm{~km} / \mathrm{h}$ and averaged between both lanes (Fig. 3), we can clearly see that the speed distribution in space and time occurring due to the $\mathrm{F} \rightarrow \mathrm{S}$ transition is qualitatively the same as that for on-ramp and off-ramp bottlenecks [Kerner, 2017].

However, rather than study a time-delayed traffic breakdown, in this paper, we illustrate, with the use of the simulation scenario (Fig. 2), the possibility and features of MB prediction based on the above general methodology. The simulations made show that qualitative results of MB prediction remain independent of simulation realizations used, the value $\mathrm{T}^{(\mathrm{B})}$, and even when the $\mathrm{MB}$ speed changes between 14.4 and $54 \mathrm{~km} / \mathrm{h}$. For this reason, the simulation scenario (Fig. 2) is used for all further simulations.

\section{Identification of Phase Transition Points on Vehicle Trajectories}

\section{Definition of Phase Transition Points on Vehicle Trajectories}

To understand the term phase transition point on a vehicle trajectory [Kerner et al., 2013], we have drawn one of the trajectories of probe vehicles in the left line (black curve labeled "trajectory of a probe vehicle" in Fig. 2). Far enough upstream of the MB the probe vehicle moves in free flow (F). 


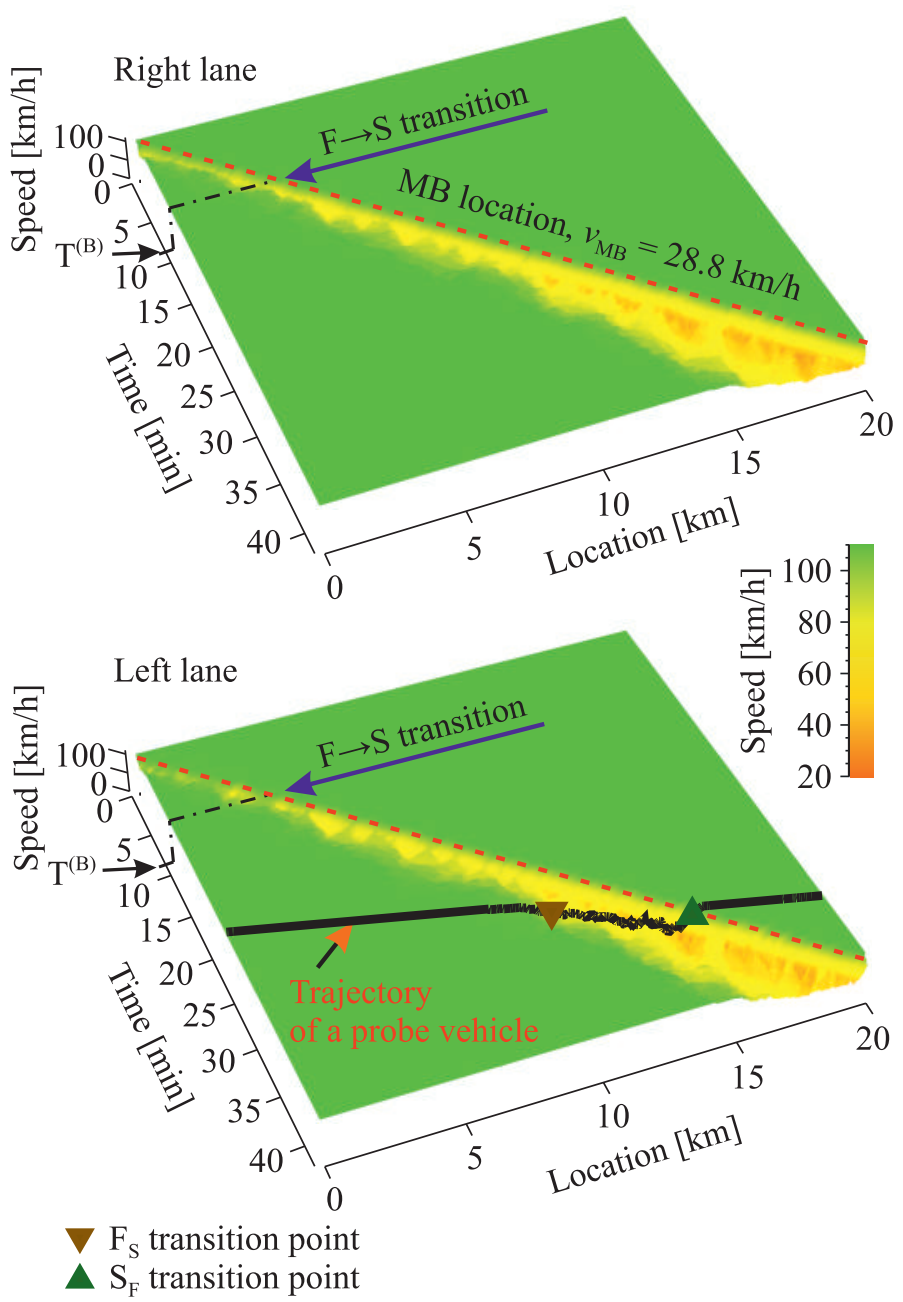

Figure 2. Simulation of traffic breakdown $(\mathrm{F} \rightarrow \mathrm{S}$ transition) at $\mathrm{MB}$. Speed data in the left and right lanes. The dotted red line marks the MB position. The model parameters are the same as those in [Kerner, Klenov, 2010, Table A2]

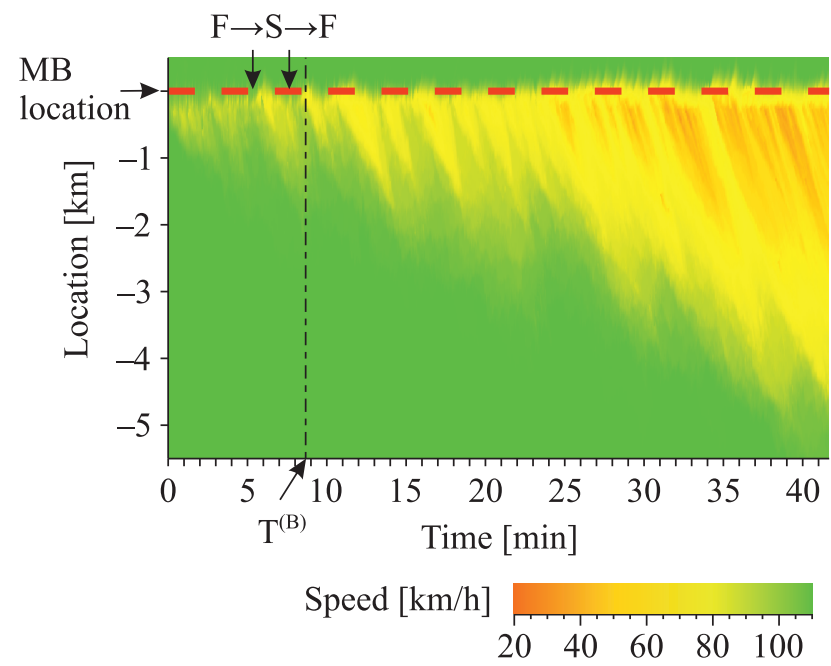

Figure 3. Simulations shown in Fig. 2 presented in the coordinate system moving at MB speed $v_{\mathrm{MB}}=28.8 \mathrm{~km} / \mathrm{h}$ (MB location is $x=0$ ). The speed data are averaged between the right and left road lanes 
While approaching the $\mathrm{MB}$, the vehicle must decelerate to a synchronized flow speed caused by the downstream front of the phase $\mathrm{S}$ at the $\mathrm{MB}$; this transition at the vehicle trajectory is called a $\mathrm{F}_{\mathrm{S}}$ phase transition point. Further, the vehicle moves through the phase $S$ upstream of the MB. Later, passing the $\mathrm{MB}$, the probe vehicle can accelerate from the phase $\mathrm{S}$ at the $\mathrm{MB}$ to the phase $\mathrm{F}$ downstream; this transition at the vehicle trajectory is called a $\mathrm{S}_{\mathrm{F}}$ phase transition point.

As can be seen in Fig. 2, a $F_{S}$ phase transition point on the vehicle trajectory (labeled by $F_{S}$ ) is different from the $\mathrm{F} \rightarrow \mathrm{S}$ transition occurring at the $\mathrm{MB}$ (labeled by $\mathrm{F} \rightarrow \mathrm{S}$ transition). Respectively, a $\mathrm{S}_{\mathrm{F}}$ phase transition point on the vehicle trajectory is not usually related to a $\mathrm{S} \rightarrow \mathrm{F}$ transition at the bottleneck. This explains different designations of $F_{S}$ phase transition point on the vehicle trajectory and the $\mathrm{F} \rightarrow \mathrm{S}$ transition at the $\mathrm{MB}$ as well as $\mathrm{S}_{\mathrm{F}}$ phase transition point on the vehicle trajectory and the $\mathrm{S} \rightarrow \mathrm{F}$ transition at the $\mathrm{MB}$.

\section{Detection of $\mathrm{F}_{\mathrm{S}}$ and $\mathrm{S}_{\mathrm{F}}$ Phase Transition Points on Vehicle Trajectories}

As explained by [Kerner et al., 2013], a probe vehicle detects automatically a phase transition point when two conditions are satisfied: (i) There is a speed change along vehicle trajectory above a chosen speed threshold and (ii) the new phase exists during a time interval that is longer than a chosen time threshold. (see Table 1). The choice of the speed and time thresholds (Table 1) proven by [Kerner et al., 2013] is as follows: The speed and time thresholds should lead to the same identification of the transitions between the phases $\mathrm{F}$ and $\mathrm{S}$ through probe vehicles as that following from the macroscopic and microscopic criteria of the traffic phases (see the criteria in Chap. 2 of [Kerner, 2009]).

Before we consider a small rate $\gamma$ of probe vehicles in the next sections, for the understanding of features of phase transition points, here we discuss the results of a study of $F_{S}$ and $S_{F}$ phase transition points along trajectories of all vehicles passing the MB. The following results have been found (Figs. 4 and 5):

1. A vehicle moves initially in the right lane (vehicle 1 in Figs. 4 and 5). While approaching the MB, vehicle 1 changes to the left lane, moving still in free flow. Then vehicle 1 identifies $F_{S}$ phase transition point in the left lane upstream of the MB. Later vehicle 1 identifies $S_{F}$ phase transition point in the left lane in a vicinity of the MB (dotted vertical line in Fig. 4).

2. A vehicle moves initially in the right lane (vehicle 2 in Figs. 4 and 5). Approaching the MB, vehicle 2 identifies $F_{S}$ phase transition point in the right lane. While moving in synchronized flow upstream of the $\mathrm{MB}$, vehicle 2 changes from the right lane to the left lane in which synchronized flow is also realized. Later vehicle 2 identifies $S_{F}$ phase transition point in the left lane in a vicinity of the MB (dotted vertical line in Fig. 4).

3. A vehicle moves initially in the left lane (vehicle 3 in Figs. 4 and 5). While approaching the MB, vehicle 3 identifies first $F_{S}$ phase transition point and later $S_{F}$ phase transition point in the left lane.

All $\mathrm{F}_{\mathrm{S}}$ and $\mathrm{S}_{\mathrm{F}}$ phase transition points along vehicle trajectories passing the MB calculated with thresholds of Table 1 are presented in Fig. 6. Below we consider $F_{S}$ and $S_{F}$ phase transition points along vehicle trajectories passing the MB (Fig. 6) regardless of the road lane in which a phase transition point has been registered by a vehicle (Fig. 7a). For simplicity, in Fig. 7b, we present the same phase transition points as those in Fig. 6a, however, in the coordinate system moving at the MB speed.

Table 1. Speed and time thresholds for phase transition point recognition

\begin{tabular}{|c|c|c|}
\hline Phase transition points & Speed threshold & Time threshold 3 \\
\hline $\mathrm{F}_{\mathrm{S}}$ phase transition point & $v<85 \mathrm{~km} / \mathrm{h}$ & $t>15 \mathrm{sec}$. \\
\hline $\mathrm{S}_{\mathrm{F}}$ phase transition point & $v>90 \mathrm{~km} / \mathrm{h}$ & $t>10 \mathrm{sec}$. \\
\hline
\end{tabular}



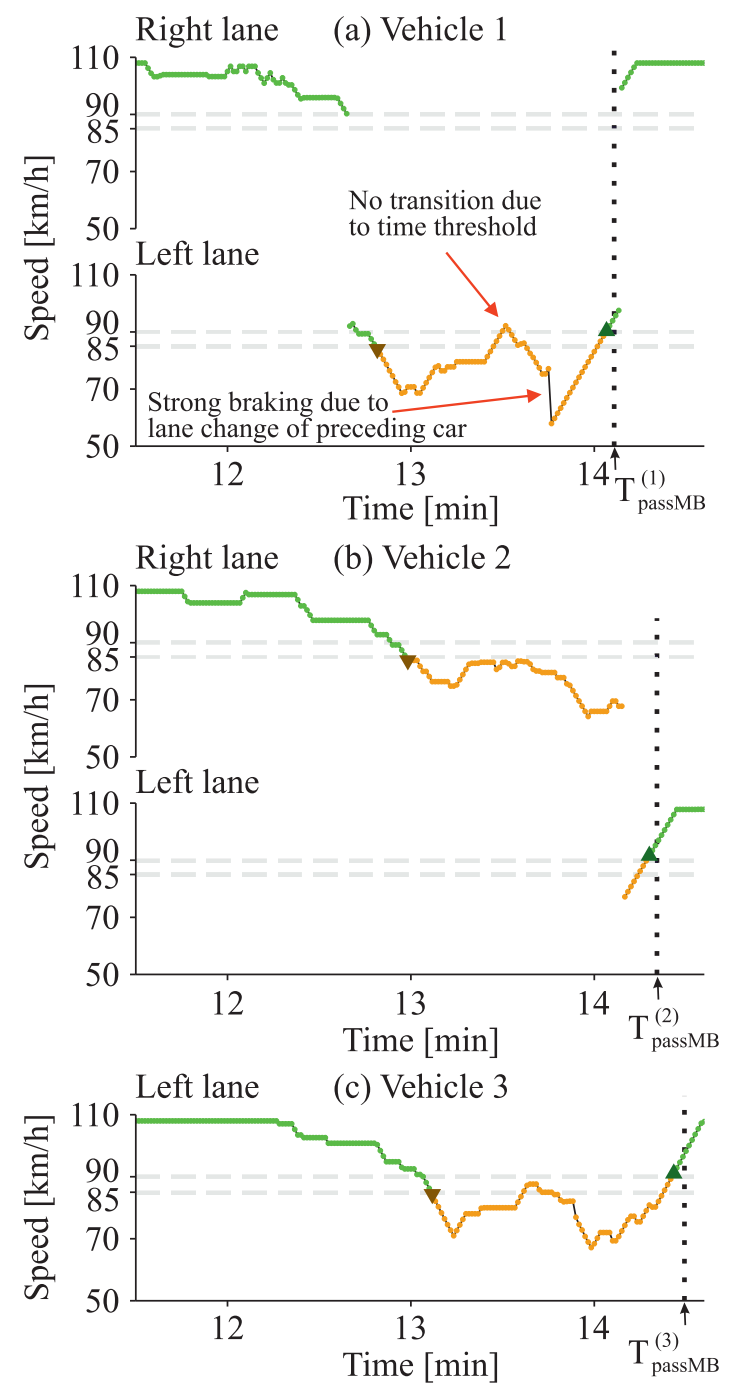

Traffic phase Phase transition points

- free flow

- synchronized flow $\checkmark \mathrm{F}_{\mathrm{S}}$ transition

$\Delta \mathrm{S}_{\mathrm{F}}$ transition

Figure 4. Phase transition points on trajectories of vehicles 1 (a), 2 (b), and 3 (c), marked by the same numbers on Fig. 5. Dotted vertical lines show time instants at which vehicles pass the MB

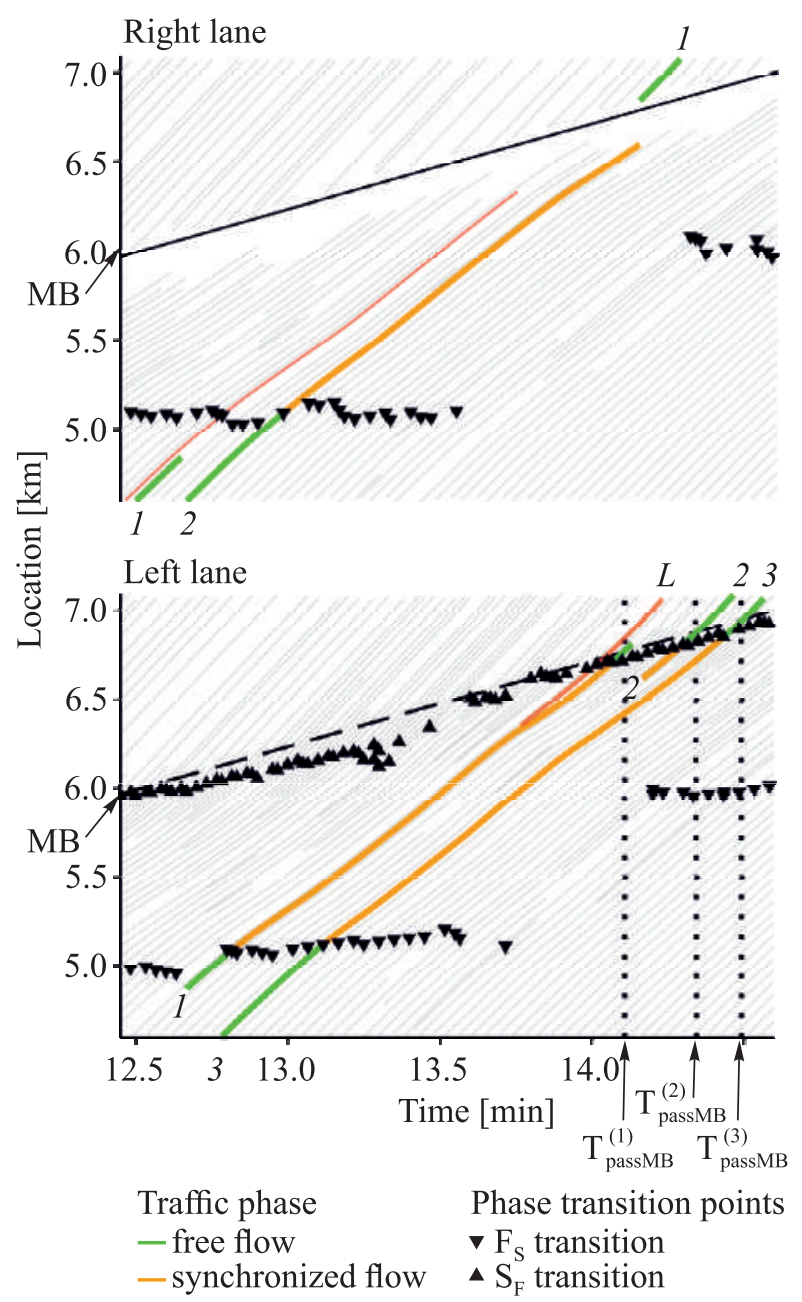

Figure 5. Trajectories of vehicles 1, 2, and 3 for which phase transition points have been studied in Fig. 4

\section{Reason for the Choice of $\mathrm{S}_{\mathrm{F}}$ Phase Transition Points for Detection of a Moving Bottleneck}

From Fig. $7(\mathrm{a}, \mathrm{b})$ we can see that the $\mathrm{F}_{\mathrm{S}}$ and $\mathrm{S}_{\mathrm{F}}$ phase transition points exhibit qualitatively different spatiotemporal behavior over time. $\mathrm{S}_{\mathrm{F}}$ phase transition points lie in the small vicinity of the location of the MB. Contrarily, the distance between locations of $F_{S}$ phase transition points and the MB location increases on average over time.

The reason for this qualitatively different behavior of $F_{S}$ and $S_{F}$ phase transition points is as follows. The downstream front of synchronized flow is localized at the MB (Fig. 3). Within the downstream synchronized flow front, synchronized flow upstream of the bottleneck transforms into free flow downstream of the bottleneck. Therefore, all vehicles register $S_{F}$ phase transition points when they are in the MB vicinity. 

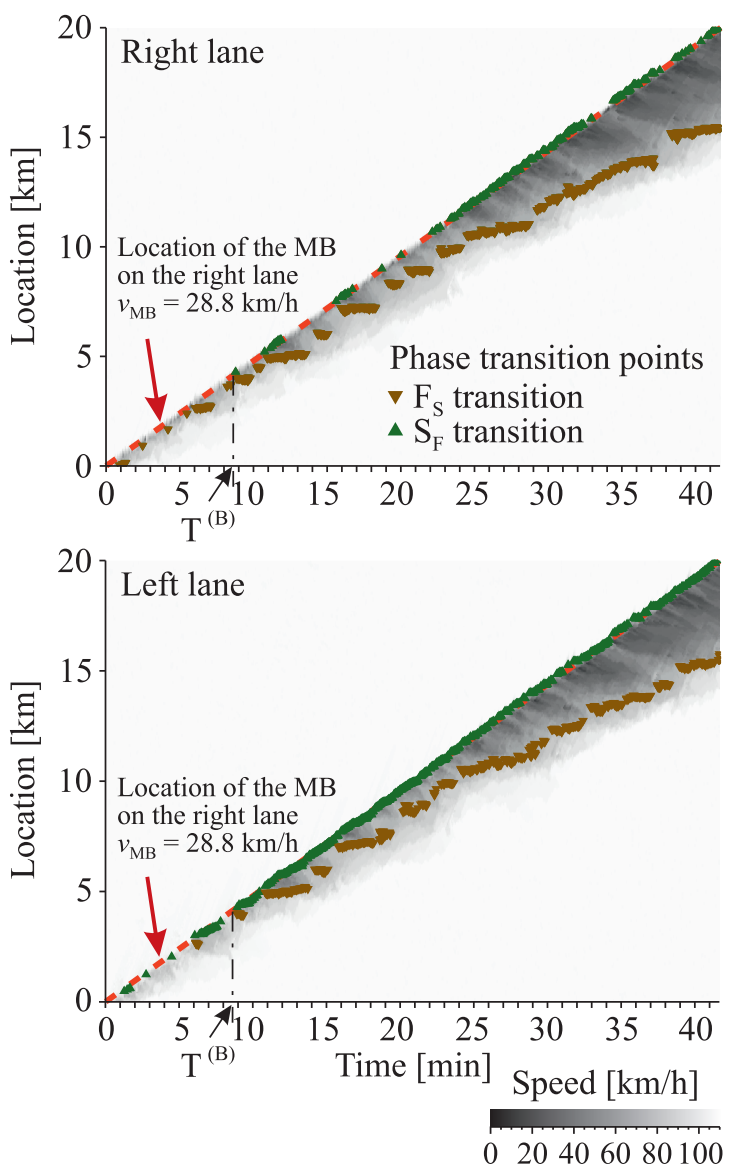

Figure 6. Phase transition points registered by all vehicles in each of the road lanes within 42 minutes of simulations in Fig. 2

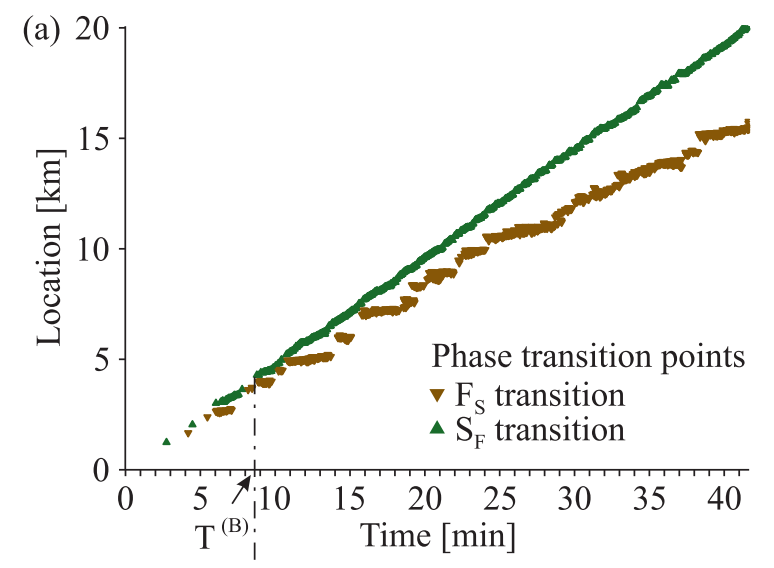

MB location
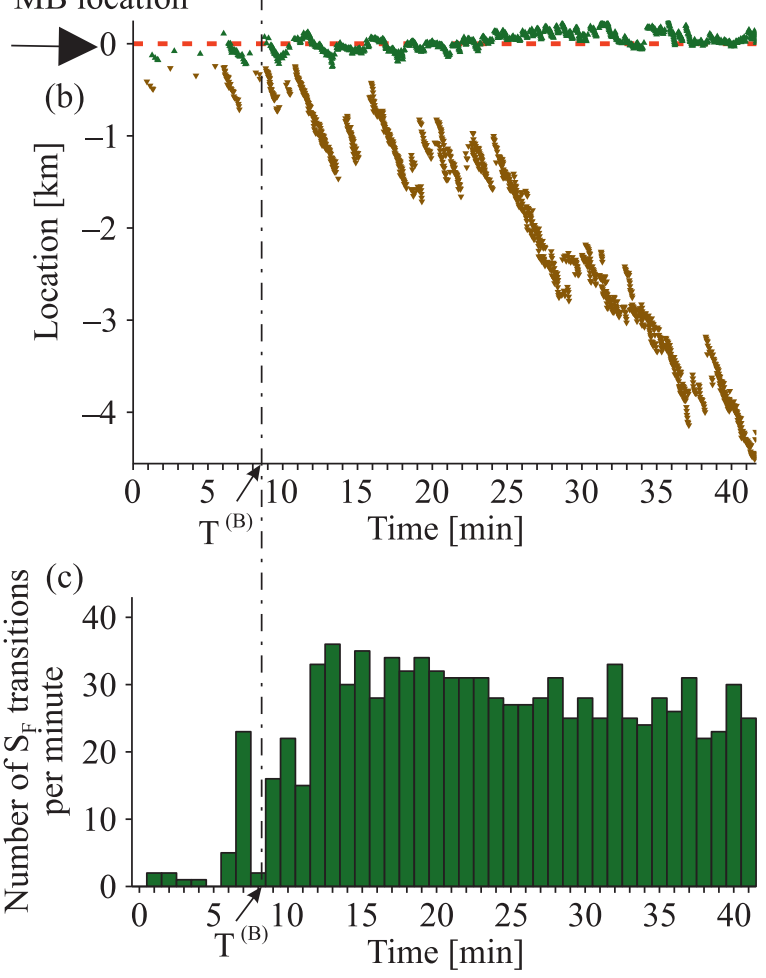

Figure $7 . \mathrm{F}_{\mathrm{S}}$ and $\mathrm{S}_{\mathrm{F}}$ phase transition points on vehicle trajectories of Fig. 6, shown regardless of the road lane. (a) motion-less coordinate system (b) coordinate system moving at MB speed. (c) Statistical analysis of $\mathrm{S}_{\mathrm{F}}$ phase transition points in $(a, b)$ : Counts of $S_{F}$ phase transition points per minute

Contrarily, the upstream synchronized flow front propagates on average upstream of the MB location. Therefore, the distance between the location of the upstream synchronized flow front and the MB location increases on average over time. Within the upstream front of synchronized flow vehicles must decelerate from a free flow speed to a synchronized flow speed. Therefore, all vehicles register $F_{S}$ phase transition points when they propagate through the upstream front of synchronized flow.

Thus, $F_{S}$ phase transition points can be far upstream of the MB location. Moreover, the distance between locations of the $\mathrm{F}_{\mathrm{S}}$ phase transition points and the MB location can be a complex time function. We can draw the following conclusions: 
- A detection of $\mathrm{F}_{\mathrm{S}}$ phase transition points is not appropriate for a reliable MB detection.

- A detection of $S_{F}$ phase transition points can be very effective for a reliable MB detection. For this reason, below only analysis of $\mathrm{S}_{\mathrm{F}}$ phase transition points will be done. Results of a statistical analysis of $S_{\mathrm{F}}$ phase transition points registered by all vehicles are shown in Fig. 7c.

\section{The effect of Kerner's $F \rightarrow S \rightarrow F$ Transitions on Detection of $\mathrm{S}_{\mathrm{F}}$ Phase Transition Points}

[Kerner, 2015] predicted that before traffic breakdown has occurred at a bottleneck, there can be a sequence of phase transitions from free flow to synchronized flow and back from synchronized flow to free flow occurring before traffic breakdown at a bottleneck. We will designate the sequence of the phase transitions as a sequence of $\mathrm{F} \rightarrow \mathrm{S} \rightarrow \mathrm{F}$ transitions at the bottleneck. The $\mathrm{F} \rightarrow \mathrm{S} \rightarrow \mathrm{F}$ transition is as follows. First, a $\mathrm{F} \rightarrow \mathrm{S}$ transition is realized at the bottleneck. The emergent synchronized flow begins to propagate upstream of the bottleneck. Then, after a short time interval a returning $\mathrm{S} \rightarrow \mathrm{F}$ transition is realized at the bottleneck. As proven in [Kerner, 2015], the return $S \rightarrow F$ transition results from an $S \rightarrow F$ instability in synchronized flow. Due to the $\mathrm{S} \rightarrow \mathrm{F}$ transition, synchronized flow dissolves and free flow returns at the bottleneck. After some random time interval, a new sequence of $\mathrm{F} \rightarrow \mathrm{S} \rightarrow \mathrm{F}$ transitions can be realized at the bottleneck, and so on. Such sequences of the $\mathrm{F} \rightarrow \mathrm{S} \rightarrow \mathrm{F}$ transitions have indeed been observed in real measured traffic data in [Molzahn et al., 2017; Duelgar et al., 2019].

Sequences of the $\mathrm{F} \rightarrow \mathrm{S} \rightarrow \mathrm{F}$ transitions occur also in our simulations (one of them is labeled by " $\mathrm{F} \rightarrow \mathrm{S} \rightarrow \mathrm{F}$ " in Fig. 3): First, an $\mathrm{F} \rightarrow \mathrm{S}$ transition is realized at the bottleneck (labeled by the left down-arrow in Fig. 3) resulting in synchronized flow propagating upstream. Then, after a short time interval of about 1.5 minutes a return $\mathrm{S} \rightarrow \mathrm{F}$ transition is realized at the bottleneck (labeled by the right down-arrow in Fig. 3).

To see sequences of the $\mathrm{F} \rightarrow \mathrm{S} \rightarrow \mathrm{F}$ transitions more clearly, in Fig. 8 (a-c) we show time-functions of vehicle speed at different road locations upstream of the MB location $x=0$. We can see the alternation of the regions of the phases $\mathrm{F}$ and $\mathrm{S}$ in Fig. 8a at $t<\mathrm{T}^{(\mathrm{B})}$ at road location $x=-0.2 \mathrm{~km}$. In [Kerner, 2015] it has been found that regions of synchronized flow caused by $\mathrm{F} \rightarrow \mathrm{S} \rightarrow \mathrm{F}$ transitions at a bottleneck first propagate upstream, widening in space and then dissolve at some distance upstream of the bottleneck. For this reason, in [Kerner, 2015] they have been called regions of dissolving synchronized flow. The same effect of dissolving synchronized flow caused by sequences of the $\mathrm{F} \rightarrow \mathrm{S} \rightarrow \mathrm{F}$ transitions is also observed at the MB: At location $x=-0.6 \mathrm{~km}$ synchronized flow regions are widening in space while propagating upstream, and then at location $x=-1.2 \mathrm{~km}$ the regions of synchronized flow have almost dissolved (Fig. 8c).

To emphasize the relevance of dissolving synchronized flow caused by sequences of the $\mathrm{F} \rightarrow \mathrm{S} \rightarrow \mathrm{F}$ transitions for MB prediction, in Fig. 8d we show a fragment of Fig. $7 \mathrm{~b}$ in which vertical dashed lines 1-12 mark regions of dissolving synchronized flow recognized by vehicles that have registered $\mathrm{F}_{\mathrm{S}}$ and $\mathrm{S}_{\mathrm{F}}$ phase transition points (labeled by triangles in Fig. 8 (d)). The same vertical dashed lines 1-12 shown in Fig. 8a prove that $\mathrm{F}_{\mathrm{S}}$ and $\mathrm{S}_{\mathrm{F}}$ phase transition points in Fig. 8d correlate with sequences of the $\mathrm{F} \rightarrow \mathrm{S} \rightarrow \mathrm{F}$ transitions shown by arrows $\mathrm{F}$ and $\mathrm{S}$ in Fig. 8a.

The importance of Kerner's $\mathrm{F} \rightarrow \mathrm{S} \rightarrow \mathrm{F}$ transitions [Kerner, 2015] for MB prediction is as follows. Synchronized flow occurring due to the $\mathrm{F} \rightarrow \mathrm{S}$ transition of a sequence of $\mathrm{F} \rightarrow \mathrm{S} \rightarrow \mathrm{F}$ transitions is registered by vehicles. Therefore, $\mathrm{S}_{\mathrm{F}}$ phase transition points associated with this synchronized flow can be used for MB prediction. However, it should be mentioned that due to the $S \rightarrow F$ transition of the sequence of $\mathrm{F} \rightarrow \mathrm{S} \rightarrow \mathrm{F}$ transitions free flow returns at the $\mathrm{MB}$ (labeled by the right down-arrow in Fig. 3). Therefore, no $S_{F}$ phase transition points can be registered. This means that due to $F \rightarrow S \rightarrow F$ transitions large time oscillations in the detection of $\mathrm{S}_{\mathrm{F}}$ phase transition points can appear. These large time oscillations in the detection of $\mathrm{S}_{\mathrm{F}}$ phase transition points on vehicle trajectories can be seen at the time interval $t<\mathrm{T}^{(\mathrm{B})}$ in Fig. $7(\mathrm{a}, \mathrm{b})$. The same effect can be found in the time function of counts of $\mathrm{S}_{\mathrm{F}}$ phase transition points (Fig. $7 \mathrm{c}$ ). 


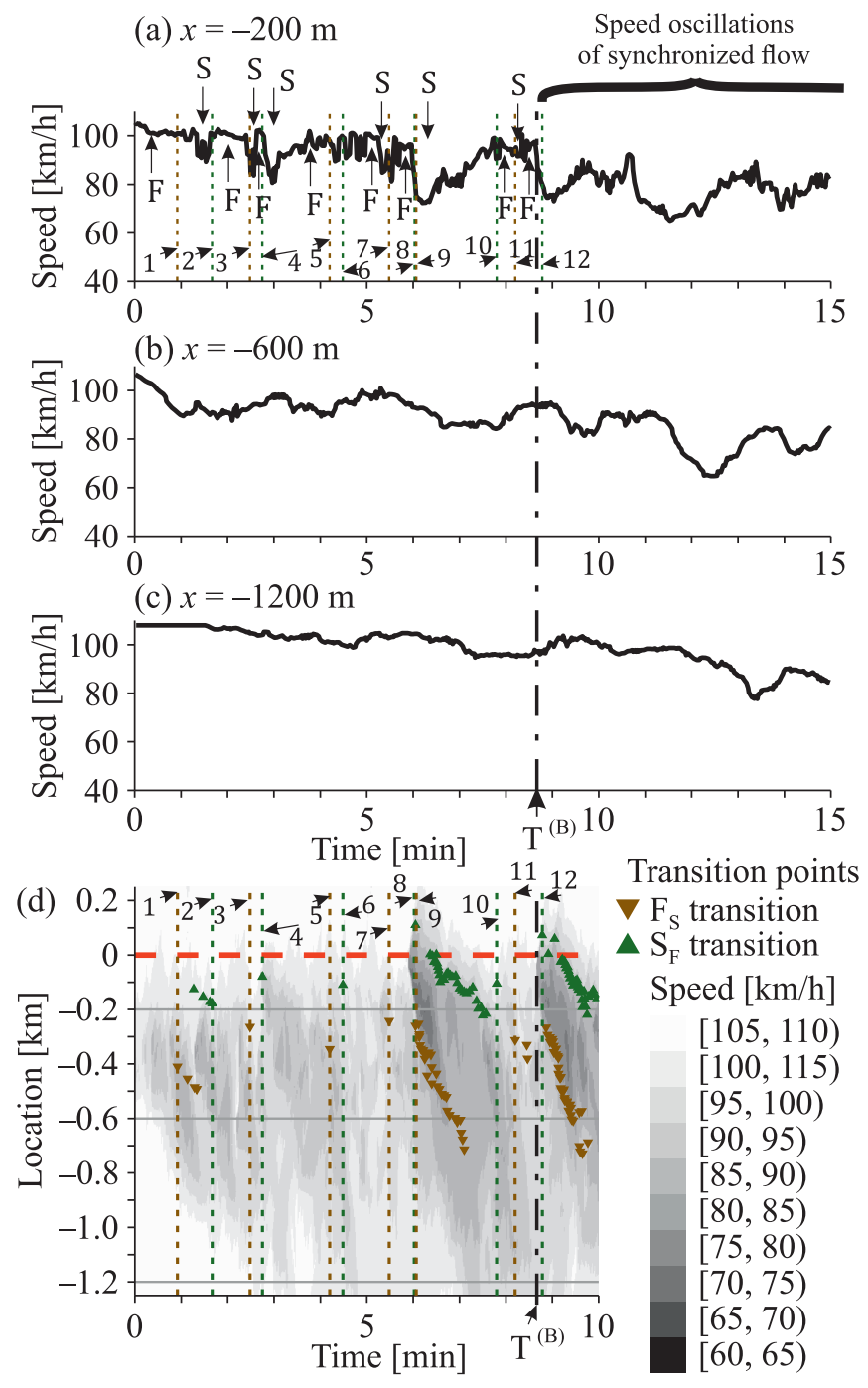

Figure 8. Continuation of Figs. 3 and 7b. (a-c) Time functions of vehicle speed (moving average over 3 vehicles) related to Fig. 3, at different distances upstream of the MB location $x=0$ in the moving coordinate system; $\mathrm{S}-$ synchronized flow, F - free flow. (d) A segment of Fig. 7b where vertical dashed lines 1-12 mark regions of dissolving synchronized flow in (a). These regions of dissolving synchronized flow are recognized by vehicles that have registered $F_{S}$ and $S_{F}$ phase transition points on vehicle trajectories; the $F_{S}$ and $S_{F}$ phase transition points are labeled by brown and green triangles, respectively

We can also see that the appearance and disappearance of synchronized flow at the MB caused by sequences of $\mathrm{F} \rightarrow \mathrm{S} \rightarrow \mathrm{F}$ transitions cause also time oscillations at the location of $\mathrm{S}_{\mathrm{F}}$ phase transition points in a vicinity of the MB location: Some of the $S_{\mathrm{F}}$ phase transition points are registered slightly upstream and others slightly downstream of the real MB location. As we will see below, this inaccuracy at locations of the $\mathrm{S}_{\mathrm{F}}$ phase transition points in comparison with the real MB location, can affect the MB prediction considerably.

In addition to sequences of $\mathrm{F} \rightarrow \mathrm{S} \rightarrow \mathrm{F}$ transitions, an inaccuracy at locations of the $\mathrm{S}_{\mathrm{F}}$ phase transition points in comparison with the real MB location is also realized after the $\mathrm{F} \rightarrow \mathrm{S}$ transition has occurred at the MB (i.e., at $t \geq \mathrm{T}^{(\mathrm{B})}$ ). The inaccuracy is associated with speed waves in synchronized flow upstream of the MB leading to complex spatiotemporal oscillations in synchronized flow speed. The speed oscillations cause the inaccuracy between locations of the $S_{F}$ phase transition points in comparison with the MB location (Fig. 7b). 
In accordance with [Kerner, 2004; Kerner, Klenov, 2010; Kerner, 2015; Kerner, 2018b], speed oscillations in synchronized flow upstream of a bottleneck are caused by dissolving speed waves in synchronized flow propagating upstream; in turn, the speed waves result from the development of speed disturbances occurring at the bottleneck. Indeed, we have found that at $t \geq \mathrm{T}^{(\mathrm{B})}$ the synchronized flow speed oscillates considerably over time (labeled by "speed oscillations of synchronized flow" in Fig. 8a at $\left.t \geq \mathrm{T}^{(\mathrm{B})}\right)$.

For the purposes of this paper it is only important to know that the random deviation of locations of $\mathrm{S}_{\mathrm{F}}$ phase transition points from MB location can have a considerable effect on the accuracy of MB prediction. For this reason, a more detailed study of other features of $\mathrm{F} \rightarrow \mathrm{S} \rightarrow \mathrm{F}$ transitions and oscillations in synchronized flow is beyond the scope of this paper.

\section{Simulations of Prediction of a Moving Bottleneck and its Reliability}

In reality, $\mathrm{S}_{\mathrm{F}}$ phase transition points can be measured by probe vehicles (FCD-vehicles) only. Currently, a share of probe vehicles is $1-2 \%$, however, it will increase over time. Below we consider characteristics of $\mathrm{MB}$ prediction with different rates $\gamma=1 \%, 2 \%, 5 \%$, and $10 \%$ of probe vehicles in traffic flow. Simulations show that at the chosen MB speed $v_{\mathrm{MB}}=28.8 \mathrm{~km} / \mathrm{h}$ the application of condition (5) allows us to identify the bottleneck as an MB; this means that there is no need for the use of condition (6).

\section{Determination of the Speed of the Moving Bottleneck}

Figure 9 shows that $\mathrm{S}_{\mathrm{F}}$ phase transition points are situated in the MB vicinity. As can be seen from Fig. 7 and explained previously, at $t<\mathrm{T}^{(\mathrm{B})} \approx 9$ min there can be $\mathrm{S}_{\mathrm{F}}$ phase transition points that are related to sequences of $\mathrm{F} \rightarrow \mathrm{S} \rightarrow \mathrm{F}$ transitions, when synchronized flow exists only for a few minutes.
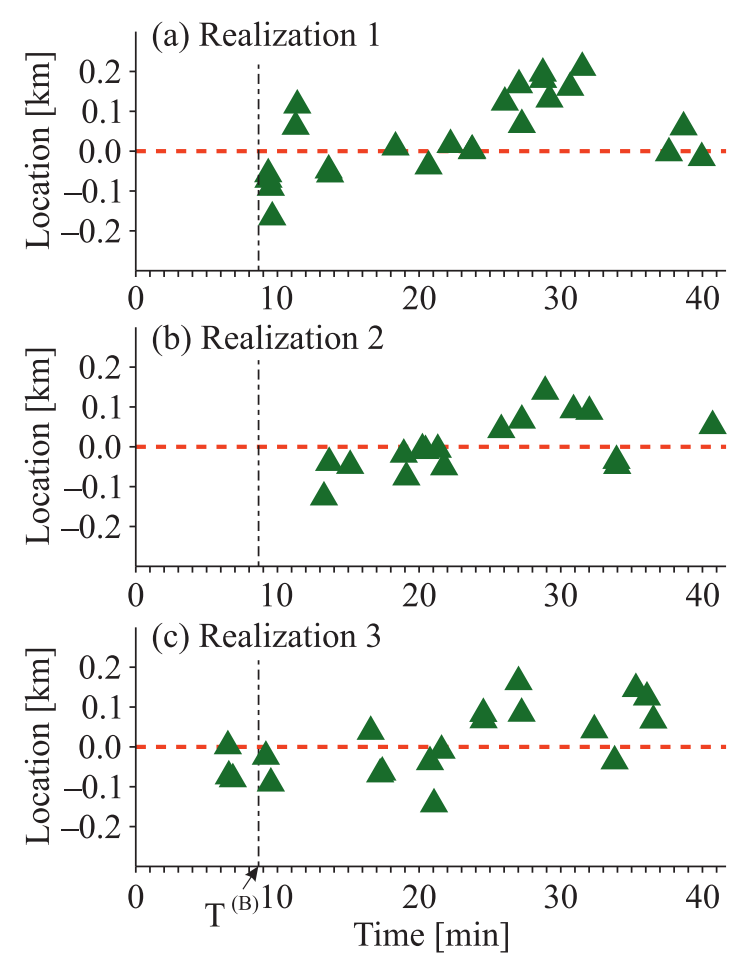

Figure 9. $\mathrm{S}_{\mathrm{F}}$ phase transition points (labeled by green bold triangles) recognized through three different random realizations of $\gamma=2 \%$ of FCD-vehicles in traffic flow presented in the coordinate system moving at MB speed: (a) realization 1 , (b) realization 2 , (c) realization 3 
For this reason, only few $\mathrm{S}_{\mathrm{F}}$ phase transition points can be registered by probe vehicles. Only after synchronized flow exists uninterruptedly at the MB that occurs at $t>\mathrm{T}^{(\mathrm{B})}$ (Fig. 7), does the recognition of $S_{\mathrm{F}}$ phase transition points by probe vehicles stabilize.

To estimate the MB speed, we apply formula (2) for each of the random realizations separately (Fig. 10). At any time instant $t$, there can be the following cases: Condition (1) is not satisfied (Fig. 10a). The estimated MB speed $v_{\text {rec }}(t)$ is negative (Fig. 10c). The estimated speed of the MB $v_{\text {rec }}(t)$ is positive (Figs. 10 (b, d-f)).

For explanation of Fig. 10, we note that, while at the time instant $t=t_{1}^{(2)}$ no conclusion about MB existence can be drawn for realization 2 (Fig. 10a), at the later time instant $t=t_{2}^{(2)}>t_{1}^{(2)}$ the second $\mathrm{S}_{\mathrm{F}}$ phase transition point has been registered for realization 2 (second triangle in Fig. 10b). The estimated MB speed (2) is $v_{\text {rec }}\left(t_{2}^{(2)}\right)=43 \mathrm{~km} / \mathrm{h}>v_{\mathrm{MB}}$ with a deviation to $v_{\mathrm{MB}}$ of $49,2 \%$.

For realization 3 (Fig. 10, c-f), at the time instant $t=t_{1}^{(3)}$ there are two $\mathrm{S}_{\mathrm{F}}$ phase transition points (two triangles in Fig. 10c). However, because the estimated MB speed (2) is negative (Fig. 10c), no conclusion about MB existence can be drawn. At the later time instant $t=t_{2}^{(3)}>t_{1}^{(3)}$ for the same realization 3 an additional $S_{\mathrm{F}}$ phase transition point has been registered (third triangle in Fig. 10d).

(a, b) Realization 2
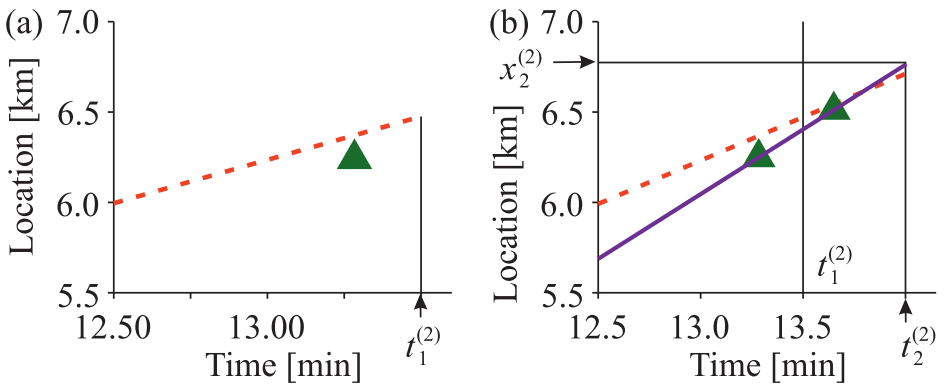

(c, f) Realization 3
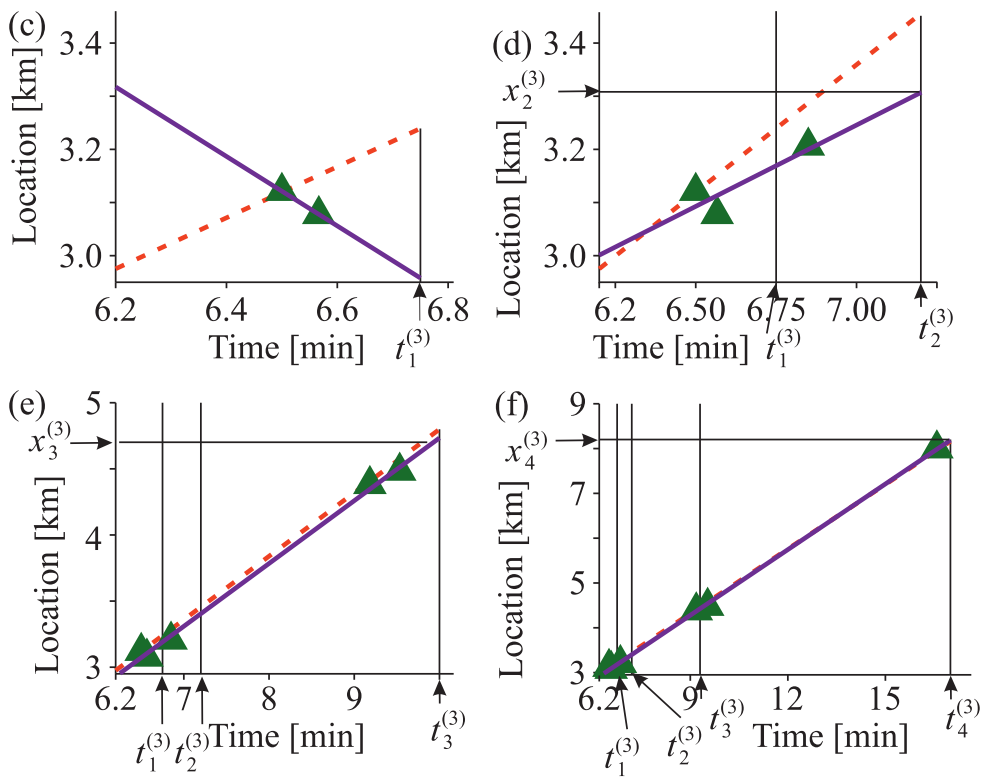

Figure 10. Estimation of the speed and location of $\mathrm{MB}$ with the use of some of $\mathrm{S}_{\mathrm{F}}$ phase transition points (labeled by triangles) in realizations 2 (a, b) and 3 (c-f) shown in Fig. 9 presented in the motion-less coordinate system. $\gamma=2 \%$. The dashed lines are related to the MB trajectory. The solid lines are related to estimated MB trajectories with different realizations at different time instants 
The estimated MB speed $v_{\text {rec }}(2)$ is now positive (realization 3 in Fig. 10d) $0<v_{\text {rec }}\left(t_{2}^{(1)}\right)<v_{\text {MB }}$ with a deviation to $v_{\mathrm{MB}}$ of $-36.3 \%$. The estimated MB speed can change considerably over time with the use of the same realization (Figs. 10, d-f). For this reason, the number of $\mathrm{S}_{\mathrm{F}}$ phase transition points $K=K_{\mathrm{BM}}$ at which condition (5) for the MB identification at a chosen confidence level $p_{\alpha}$ is satisfied can be different for different realizations.

An example of the MB speed estimation over time in one of the realizations is shown in Fig. 11. In the realization two first $\mathrm{S}_{\mathrm{F}}$ phase transition points (labeled by $K=2$ in Fig. 11) lead to a negative value of the speed $v_{\text {rec }}$ for both chosen confidence levels $p_{\alpha}=0.9$ and 0.99 . Later, when the next $\mathrm{S}_{\mathrm{F}}$ phase transition points have been detected, the speed $v_{\text {rec }}$ becomes positive, however, $K<K_{\mathrm{MB}}$ : condition (5) is not satisfied continuously over time. Finally, at $K=K_{\mathrm{MB}}=5$ condition (5) is satisfied for any time $t \geq t_{\mathrm{BM}}=677 \mathrm{sec}$. Therefore, in the realization the bottleneck has been identified as an MB at the same value $K=K_{\mathrm{MB}}=5$ for both chosen confidence levels $p_{\alpha}=0.9$ and 0.99 .

We have found that for some other realizations the value $K=K_{\mathrm{MB}}$ can increase with the increase in the confidence level. In general, the value $K=K_{\mathrm{MB}}$ can depend both on a chosen confidence level and on rates $\gamma$ of FCD-vehicles as shown in a statistical analysis of condition (5) presented in Fig. 12.

For simulations of probability $P_{\mathrm{MB}}(t)$ of MB prediction with formula (7) (Fig. 13), we choose the number of random realizations $M=1000$. This choice is as follows. The larger the value $M$ in (7), the more precise the statistical analysis of the reliability of MB prediction. However, the larger $M$, the longer the calculation time. Simulations show that at $M=1000$ calculations of any statistical value of the reliability of MB prediction are made with an accuracy better than $1 \%$ at the appropriate short time of simulations.

It is clear that the probability $P_{\mathrm{MB}}(t)$ of $\mathrm{MB}$ recognition (7) grows over time and at a given time instant $t$ the probability $P_{\mathrm{MB}}$ is the larger the larger the rate $\gamma$ of FDC-vehicles (Fig. 13). Moreover, for each given value of $\gamma$ the probability $P_{\mathrm{MB}}(t)$ decreases with an increase in the chosen confidence levels $p_{\alpha}$ (Fig. 13). However, we note an important feature of these time functions of the probability $P_{\mathrm{MB}}(t)$ : There are some time intervals on each of the curves $P_{\mathrm{MB}}(t)$ within which the probability $P_{\mathrm{MB}}(t)$ does not increase over time (one of these time intervals is labeled by " $\mathrm{S} \rightarrow \mathrm{F}$ "

(a) Realization $1, p_{\alpha}=0.9$

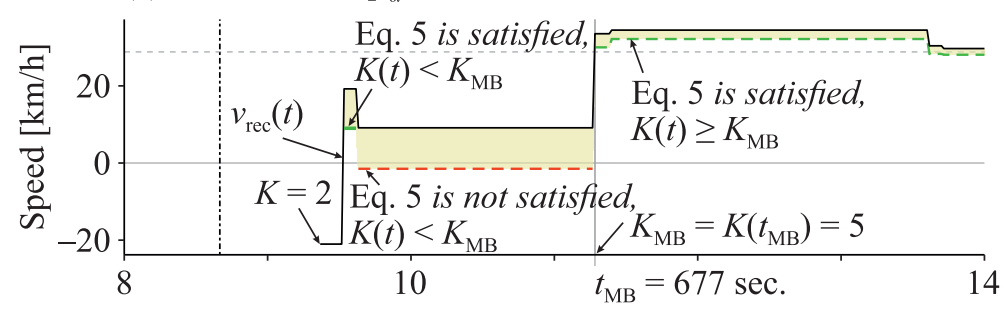

(b) Realization $1, p_{\alpha}=0.99$

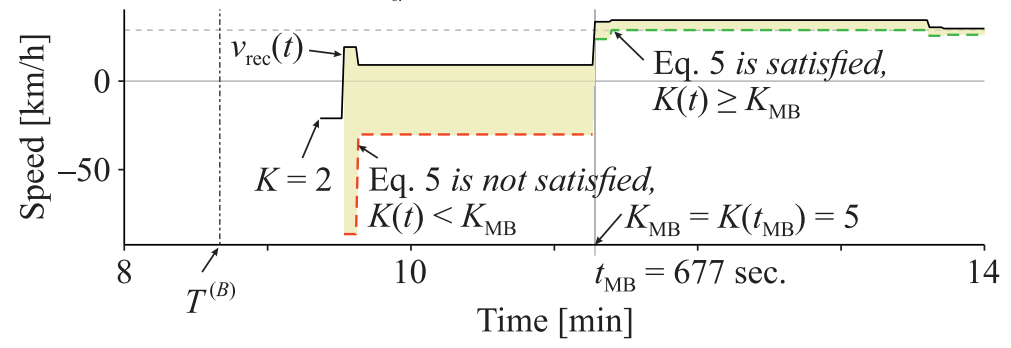

Figure 11. Time dependence of MB speed $v_{\text {rec }}(t)$ and its comparison with time dependence with the term $t_{\alpha, K(t)-2}^{(1)} \sigma_{\text {rec }}(K(t))$ in condition (5) for realization 1 shown in Fig. 9a. (a) $p_{\alpha}=0.9$. (b) $p_{\alpha}=0.99$. The solid curve is related to $v_{\mathrm{rec}}(t)$. The dashed curves are related to $\Delta v=v_{\mathrm{rec}}(t)-t_{\alpha, K(t)-2}^{(1)} \sigma_{\mathrm{rec}}(K(t))$ (red curves for $\Delta v<0$ and blue curves for $\Delta v>0$ ) 

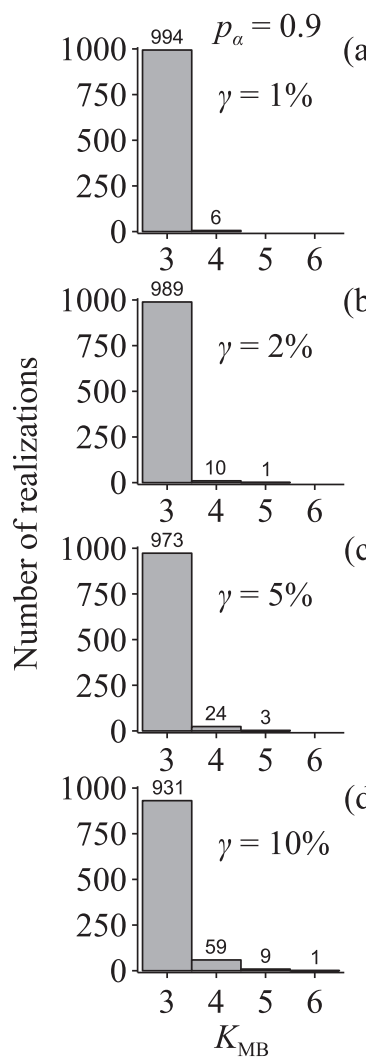

(b)
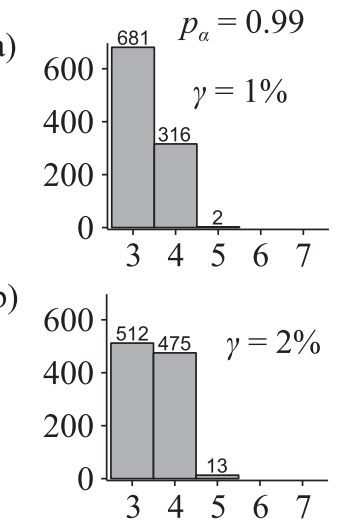

(c)

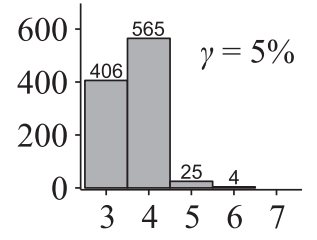

(d)

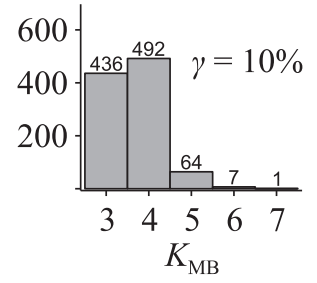

Figure 12. Histograms for the number of realizations versus values $K=K_{\mathrm{BM}}$ at which condition (5) is satisfied. The left and right columns are related to the confidence levels $p_{\alpha}=0.9$ and 0.99 , respectively. (a) $\gamma=1 \%$. (b) $\gamma=2 \%$. (b) $\gamma=5 \%$. (c) $\gamma=10 \%$. In the statistical analysis all $M=1000$ different realizations have been used
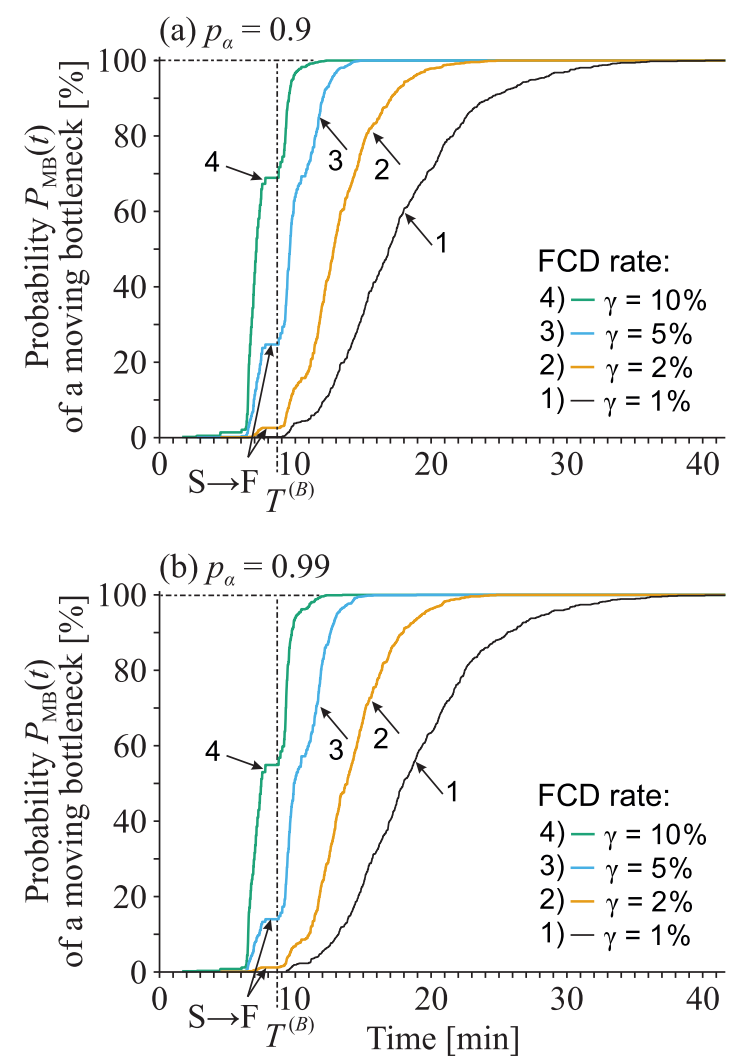

Figure 13. Probability $P_{\mathrm{MB}}(t)$ of $\mathrm{MB}$ recognition found from (7) for two different confidence levels $p_{\alpha}=0.9$ (a) and 0.99 (b), which have been calculated for different rates $\gamma$ of FCD-vehicles: Curve 1 for $\gamma=1 \%$, curve 2 for $\gamma=2 \%$, curve 3 for $\gamma=5 \%$ and curve 4 for $\gamma=10 \%$

on curves 1 for $\gamma=1 \%$ in Fig. 13). This behavior of time functions $P_{\mathrm{MB}}(t)$ is associated with $\mathrm{F} \rightarrow \mathrm{S} \rightarrow \mathrm{F}$ transitions. When an $\mathrm{S} \rightarrow \mathrm{F}$ transition within a sequence of the $\mathrm{F} \rightarrow \mathrm{S} \rightarrow \mathrm{F}$ transitions is realized, free flow returns at the MB. As long as free flow exists at the $\mathrm{MB}$, no $\mathrm{S}_{\mathrm{F}}$ phase transition points can occur at vehicle trajectories passing the bottleneck. Therefore, the probability $P_{\mathrm{MB}}(t)$ of $\mathrm{MB}$ recognition cannot increase over time within the time interval in which free flow persists at the MB. When later the subsequent $\mathrm{S} \rightarrow \mathrm{F}$ transition of a sequence of the $\mathrm{F} \rightarrow \mathrm{S} \rightarrow \mathrm{F}$ transitions causes synchronized flow at the MB, the probability $P_{\mathrm{MB}}(t)$ begins to increase over time as long as synchronized flow persists at the MB, and so on. The larger the rate $\gamma$ of FCD-vehicles, the more frequently the sequences of the $\mathrm{F} \rightarrow \mathrm{S} \rightarrow \mathrm{F}$ transitions affect a time function of the probability $P_{\mathrm{MB}}(t)$. For this reason, there are many short time intervals for which no growth in the time function of the probability $P_{\mathrm{MB}}(t)$ for the FCD-vehicle rate $\gamma=10 \%$ occurs (curves 4 in Fig. 13).

\section{Estimation of the Location of a Moving Bottleneck}

The estimation of MB location $x_{\text {rec }}(t)$ (3) is shown in Fig. 10 (b, d-f). For realization 3 in Fig. 9c, three estimated MB locations $x_{\text {rec }}(t)=x_{k}^{(3)}(t)$ related to time instants $t=t_{k}^{(3)}, k=2,3,4$ are shown in Figs. $10(\mathrm{~d}-\mathrm{f})$, respectively. The time dependence of the estimated MB location (3) for realization 3 in Fig. 9c is presented in Fig. 14a. 

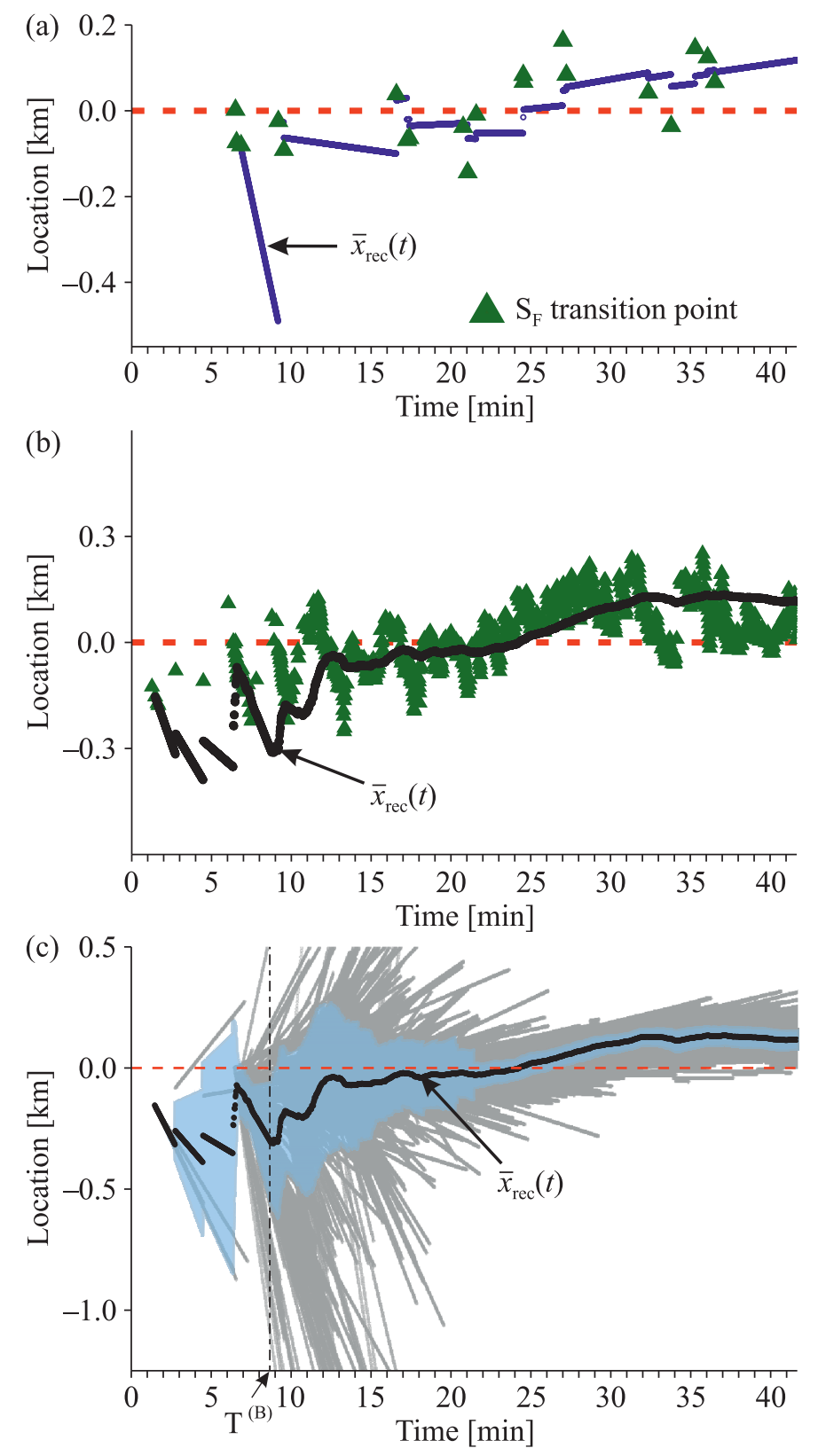

Figure 14. Estimated MB locations and their statistical errors presented in the coordinate system moving at MB speed (MB location is equal to $x=0$ ): (a) $x_{\text {rec }}(t)$ for realization 3 shown in Fig. 9c. (b, c) $\bar{x}_{\text {rec }}(t)$ for $M=1000$ realizations calculated with (9). In $(a, b)$, triangles mark locations of $S_{F}$ phase transition points in MB vicinity. In (c), the gray points show $x_{\text {rec }}(t)$ for all $M=1000$ realizations, whereas the blue area indicates the standard deviation from $\bar{x}_{\text {rec }}(t)$

The appearance and disappearance of synchronized flow at the MB caused by sequences of $\mathrm{F} \rightarrow \mathrm{S} \rightarrow \mathrm{F}$ transitions and speed oscillations in synchronized flow (Fig. 8) cause considerably time oscillations at locations of $S_{F}$ phase transition points in a MB vicinity: Some of the $S_{F}$ phase transition points are registered slightly upstream and others slightly downstream of the real MB location $x=0$ (Figs. $14(\mathrm{a}, \mathrm{b})$ ). The deviation of the mean estimated MB location $\bar{x}_{\mathrm{rec}}(t)(9)$ shown in Figs. 14 (b, c) from the real MB location $x=0$ emphasizes statistical errors caused by sequences of $\mathrm{F} \rightarrow \mathrm{S} \rightarrow \mathrm{F}$ transitions (at $t<\mathrm{T}^{(\mathrm{B})}$ ) and speed oscillations in synchronized flow (at $t \geq \mathrm{T}^{(\mathrm{B})}$ ). 


\section{The Advanced Method of Prediction of an Unexpected Bottleneck Caused by Stopped Vehicle (SV)}

\section{Methodology of on-Line Prediction of an SV Bottleneck}

A methodology for predicting an SV bottleneck caused by a stopped vehicle (SV) (Fig. 15) that can be used for on-line applications is as follows:

1. We assume a random distribution of FCD-vehicles with rate $\gamma$ between other vehicles that in simulations is called a random realization (or a sample) of FCD-vehicles.

2. For each time instant we prove whether FCD-vehicles in the realization have registered $\mathrm{S}_{\mathrm{F}}$ phase transition points (from the phase $\mathrm{S}$ to the phase $\mathrm{F}$ ).

3. SV bottleneck prediction is only possible if at some time instant $t$ there are at least two $\mathrm{S}_{\mathrm{F}}$ phase transition points, i.e., if condition (1) is satisfied.

4. To estimate the bottleneck speed, we apply a standard linear regression through the points of the $\mathrm{S}_{\mathrm{F}}$ phase transition points for each of the $M=1000$ different random realizations (samples) (Fig. 10). Under condition (1), i.e., we assume that at time instant $t$ there are at least two $\mathrm{S}_{\mathrm{F}}$ phase transition points registered by FCD-vehicles in a random realization, we estimate the bottleneck speed with the use of condition (2).

5. Then the bottleneck location denoted by $x_{\text {rec }}$ can be estimated with the use of condition (3).

6. The standard error for the estimated bottleneck speed $v_{\text {rec }}$ is given by (4).

7. Using the estimated bottleneck speed $v_{\text {rec }}(K)$ (2) and standard error $\sigma_{\text {rec }}(4)$, one can find the condition that the bottleneck considered occurs because of a stopped vehicle, i.e., the real speed of the bottleneck is zero.

8. With the use of conditions (6), we can estimate whether at the confidence level $p_{\alpha}$ the bottleneck speed can be considered to be close to zero. In (6) $\Delta_{\mathrm{SV}}>0$ is a constant (e.g., $\Delta_{\mathrm{SV}}=2 \mathrm{~km} / \mathrm{h}$ ), the value $t_{\alpha, K-2}^{(2)}$ is found from Student's t-distribution with $K-2$ degrees of freedom for the given confidence level $p_{\alpha}$, the parameter $\alpha$ is $\alpha=1-p_{\alpha}$. We denote $K_{\mathrm{SV}}$ as the value $K=K\left(t_{\mathrm{SV}}\right)$ at which condition (6) is satisfied for any time $t \geq t_{\mathrm{SV}}$.

9. The value $t_{\alpha, K-2}^{(2)}$ is determined as follows: If a random variable $T$ is distributed according to Student's t-distribution with $K-2$ degrees of freedom, then a value of $T$ lies within the twosided region $-t_{\alpha, K-2}^{(2)}<T<t_{\alpha, K-2}^{(2)}$ with the probability $p_{\alpha}=1-\alpha$. The value $t_{\alpha, K-2}^{(2)}$ at the given values $\alpha$ and $K-2$ can be found from t-distribution tables for a two-sided region ${ }^{1}$.

10. When conditions (6) are satisfied at $K=K_{\mathrm{SV}}$, one can conclude that an SV bottleneck is identified at the confidence level $p_{\alpha}$. To explain the above definition of $K_{\mathrm{SV}}$, we note that if (6) has been satisfied for some value of $K$, condition (6) should also be checked for any larger values $K(t)$. If at some larger $K(t)$ condition (6) becomes invalid, the old value $K_{\mathrm{SV}}$ should be removed from calculations and a new value $K=K_{\mathrm{SV}}$ should be found, for which condition (5) is satisfied at the confidence level $p_{\alpha}$. Then the condition (5) should be checked further for larger values $K(t)$.

11. As the value of $K$ increases further with time, conditions (6) should also be checked for values $K(t)>K_{\mathrm{SV}}$. If at some $K(t)>K_{\mathrm{SV}}$ conditions (6) become invalid, the next value $K_{\mathrm{SV}}$ should be found for which conditions (6) are satisfied at the confidence level $p_{\alpha}$. Then conditions (6) should be checked further for values $K(t)$ larger than the found value $K_{\mathrm{SV}}$.

\footnotetext{
${ }^{1}$ See Internet site https://en.wikipedia.org/wiki/Student27s_t-distribution\#Table_of_selected_values.
} 


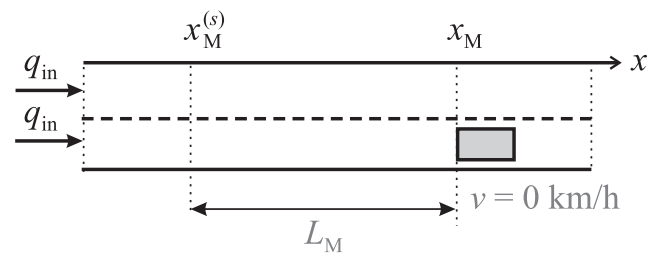

Figure 15. Schematic representation of a scenario with a stopped vehicle (SV) in the right lane of the road

12. It can be seen in Figs. 16 and 17 that $S_{F}$ phase transition points are located in the bottleneck vicinity. Therefore, locations of $S_{\mathrm{F}}$ phase transition points registered by probe vehicles following each other should not move on average for the SV bottleneck. This allows us to draw the following conclusions:

(i) If at time instant $t=t_{\mathrm{SV}}$ conditions (6) have been satisfied, we can state at the confidence level $p_{\alpha}$ that there is a bottleneck caused by a stopped vehicle in traffic flow.

(ii) The location $x_{\mathrm{rec}}(t)$ of the SV bottleneck can be estimated according to formula (3) at $v_{\text {rec }}=0$.

13. Bottleneck speed (2) and location (3) of the SV bottleneck are sent to automated driving vehicles.

14. It can turn out that, due to possible large errors at locations of $\mathrm{S}_{\mathrm{F}}$ for the SV bottleneck, conditions (6) are not satisfied. Then we cannot state that there is an SV bottleneck caused by a stopped vehicle in traffic flow.

15. Because initially we do not know what kind of a bottleneck is related to a sequence of $S_{F}$ phase transition points registered by FCD-vehicles, in a general case, we should prove conditions (6) and (5) simultaneously. If condition (5) is satisfied, then at the confidence level $p_{\alpha}$ we can state that the bottleneck is an MB. Contrarily, if condition (6) is satisfied, then at the confidence level $p_{\alpha}$ we can state that the bottleneck is an SV bottleneck.

16. Additionally, when condition (6) is satisfied, we should prove that there are no possible road bottlenecks (e.g., on- or off-ramps) in the digital map at the estimated location of the SV bottleneck. Otherwise, it is more probable that the bottleneck related to a sequence of $\mathrm{S}_{\mathrm{F}}$ phase transition points registered by FCD-vehicles is really in a road bottleneck rather than it is caused by a stopped vehicle.

\section{Probability of Prediction of a Stopped Vehicle (SV)}

The reliability of the above methodology of on-line prediction of an SV bottleneck can be found from the following statistical off-line data analysis:

- We repeat items of the above methodology for each of a large number $M$ (where $M \gg 1$ ) different random realizations.

- We calculate the total number of realizations $m_{\mathrm{SV}}(t)$ (where $m_{\mathrm{SV}}(t) \leq M$ ) in which conditions (6) have been satisfied at the confidence level $p_{\alpha}$ at the time instant $t$. The number of opposite cases, in which conditions (6) have not been satisfied, is equal to $M-m_{\mathrm{SV}}(t)$.

- Then the probability $P_{\mathrm{SV}}(t)$ that at time instant $t$ a bottleneck caused by a stopped vehicle is identified at the confidence level $p_{\alpha}$ is

$$
P_{\mathrm{SV}}(t)=\frac{m_{\mathrm{SV}}(t)}{M}
$$




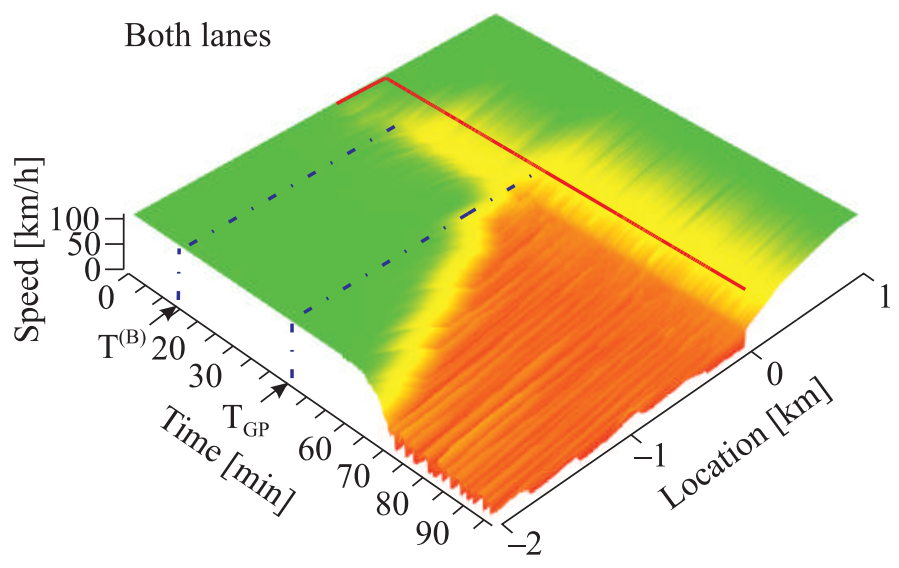

Figure 16. Presentation of simulation results: speeds of all simulated vehicles, but without the stopped vehicle (SV), averaged over both lanes as a three-dimensional color plot versus time and space. The red curve shows the location of the stopped vehicle from the moment the vehicle starts braking. For the analysis, the origin of the local axis was placed at the road location at which the vehicle has come to a standstill. The temporal observation begins 30 seconds before the vehicle begins to brake

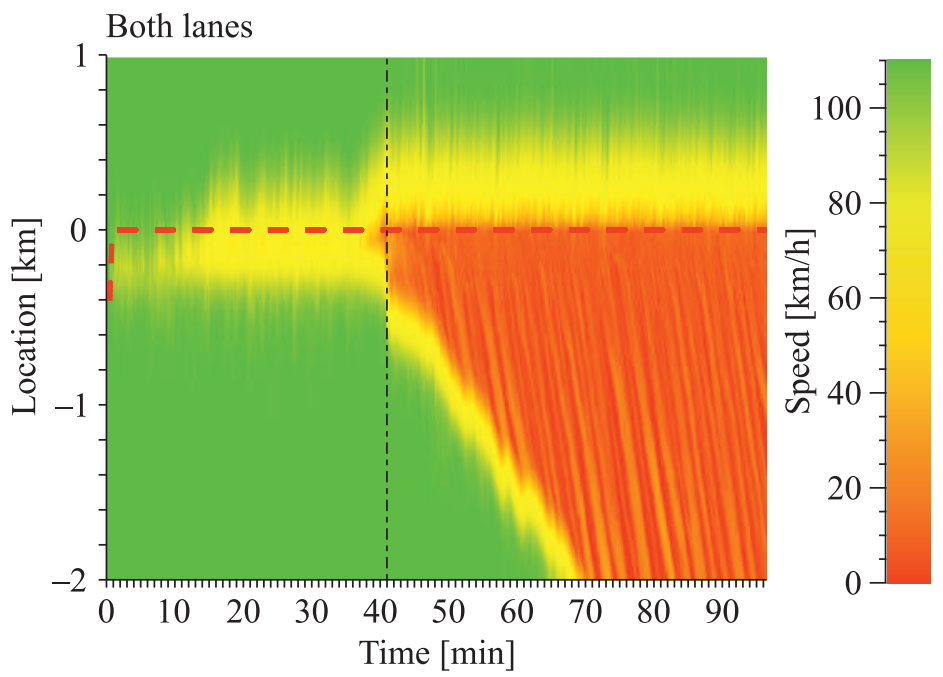

Figure 17. Continuation of Fig. 16. Two-dimensional representation of the simulation result as a color-coded speed (averaged over both road lanes) versus road location and time

- It can occur that in one of the realizations conditions (6) are satisfied at the confidence level $p_{\alpha}$ at some time instant $t_{\mathrm{SV}}$ at the number of points $K_{\mathrm{SV}}=K\left(t_{\mathrm{SV}}\right)$, but conditions (6) become invalid at some later time instant $t_{\mathrm{no}}$ at the number of points $K\left(t_{\mathrm{no}}\right)>K_{\mathrm{SV}}$. In this case it is supposed that a bottleneck caused by a stopped vehicle is not identified in this realization at $t \leq t_{\text {no }}$. Therefore, the value $m_{\mathrm{SV}}(t)$ in (10) should be diminished by 1 within the time interval $t_{\mathrm{SV}} \leq t \leq t_{\mathrm{no}}$.

- We repeat this calculation of $P_{\mathrm{SV}}(t)$ for different rates $\gamma$ of probe vehicles.

- We study errors at the estimated bottleneck location. We calculate the mean location of the SV bottleneck $\bar{x}_{\text {rec }}(t)$ averaged over all $M$ realizations with the use of formula (9). 


\section{Scenario for a Stopped (Broken-Down) Vehicle}

The model of traffic at the SV bottleneck in the right lane follows from the model of traffic with an $\mathrm{MB}$, if the $\mathrm{MB}$ speed is set to zero: $v_{\mathrm{MB}}=0$. The same general conditions have been applied as those in the scenario with the MB. Due to the SV in the right lane, all other vehicles that drive first in the right lane must change to the left lane before the vehicles have reached the SV. In the model, there are special lane changing rules in the area 300 meters before the SV location (for these lane changing rules, see in Appendix A of the book [Kerner, 2017]).

For the SV scenario, the flow rate $q_{\text {in }}=1259$ vehicles/(h lane) is used which is lower than that for the MB scenario. The simulation time is 100 minutes. At a random time instant, a vehicle in the simulation starts to decelerate with the deceleration $b=1 \mathrm{~m} / \mathrm{sec}^{2}$ until it comes to a complete stop. Results of the simulations can be seen in Figs. 16 and 17. In these figures, speeds of all simulated vehicles (but without the SV) are shown. The vehicle speeds are averaged over both road lanes as a three-dimensional colored plot of time and space. The red curve shows the position of the stopped vehicle from the moment it starts braking. For the analysis, the origin of the local axis was placed at the position at which the vehicle has come to a standstill. The chronological observation begins 30 seconds before the vehicle happens to start braking.

\section{Traffic Breakdown at SV Bottleneck}

One can see that after a (random) delay time $\mathrm{T}^{(\mathrm{B})}$ a traffic breakdown occurs at the SV bottleneck (Figs. 16 and 17). The traffic breakdown causes the formation of complex spatiotemporal congested patterns at the SV bottleneck. First, after the traffic breakdown a localized synchronized flow pattern (LSP) emerges at the SV bottleneck. The LSP exists for a long time interval.

After this time interval, at some time instant $t=\mathrm{T}_{\mathrm{GP}}$, the LSP transforms into a general congested traffic pattern (GP). The GP consists of two phases $\mathrm{S}$ and $\mathrm{J}$ of the congested traffic. In accordance with Kerner's three-phase traffic theory, the transformation of the LSP into the GP occurs spontaneously due to an $\mathrm{S} \rightarrow \mathrm{J}$ phase transition in synchronized traffic of the LSP. The physics of the $\mathrm{S} \rightarrow \mathrm{J}$ phase transition and such a transformation of an LSP into the GP has been described in Chap. 12.5.3 and Fig. 20.7 of the book [Kerner, 2004].

\section{Localized Synchronized Flow Pattern (LSP)}

To see that traffic congestion that arises due to traffic breakdown occurring after a delay time $\mathrm{T}^{(\mathrm{B})}$ is really the LSP, we will first look at the structure of congested traffic in Figs. 16 and 17 during the time interval $0 \leq t<\mathrm{T}_{\mathrm{GP}}$ (Figs. 18, 19 and 20).

We can see that the vehicle speed as a function of time at the location of the SV (Fig. 20b) at time instant $t=\mathrm{T}^{(\mathrm{B})}$ suddenly drops: Traffic breakdown ( $\mathrm{F} \rightarrow \mathrm{S}$ transition) has occurred.

Synchronized flow traffic phase resulting from traffic breakdown ( $\mathrm{F} \rightarrow \mathrm{S}$ transition) propagates only a few hundred meters upstream from the SV (Fig. 20, c-f). Indeed, 400 meters upstream of the $\mathrm{SV}$ free flow is realized (Fig. 20g). In other words, the expansion of synchronized flow upstream of the SV bottleneck is limited. That is the main characteristic feature of the LSP.

The reason for the LSP formation after a traffic breakdown at the SV is as follows: A traffic breakdown ( $\mathrm{F} \rightarrow \mathrm{S}$ transition) caused by the SV can occur at a smaller flow rate $q_{\text {in }}=1259$ vehicles $/ \mathrm{h}$ per lane as the flow rate $q_{\text {in }}=1375$ vehicles/h per lane, which has been used in the case when traffic breakdown has occurred at the MB. Because of the smaller flow rate $q_{\text {in }}=1259$ vehicles $/ \mathrm{h}$ (per lane) the expansion of synchronized flow upstream of the SV severely limited. This explains the LSP occurrence at the SV bottleneck. 


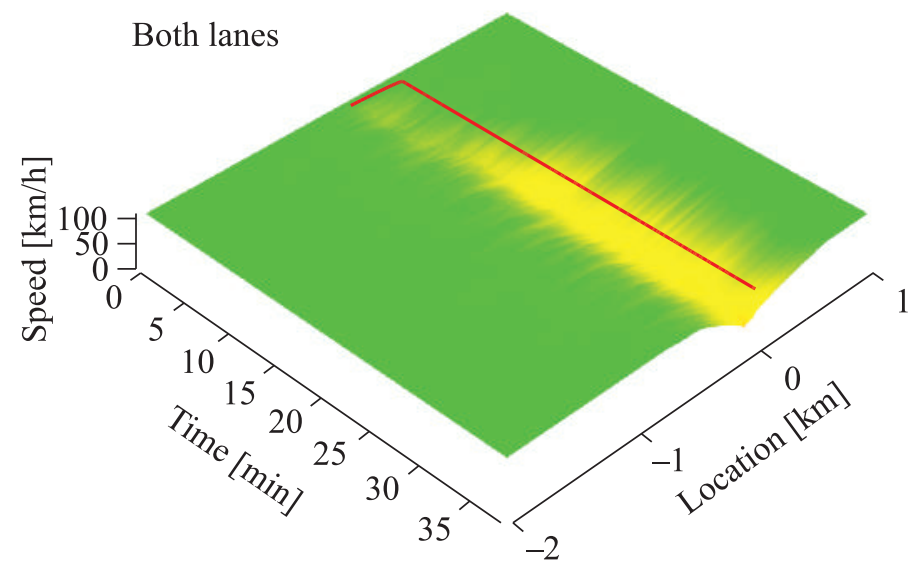

Figure 18. Representation of the simulation results of Fig. 16 in the time interval $0 \leq t<\mathrm{T}_{\mathrm{GP}}$

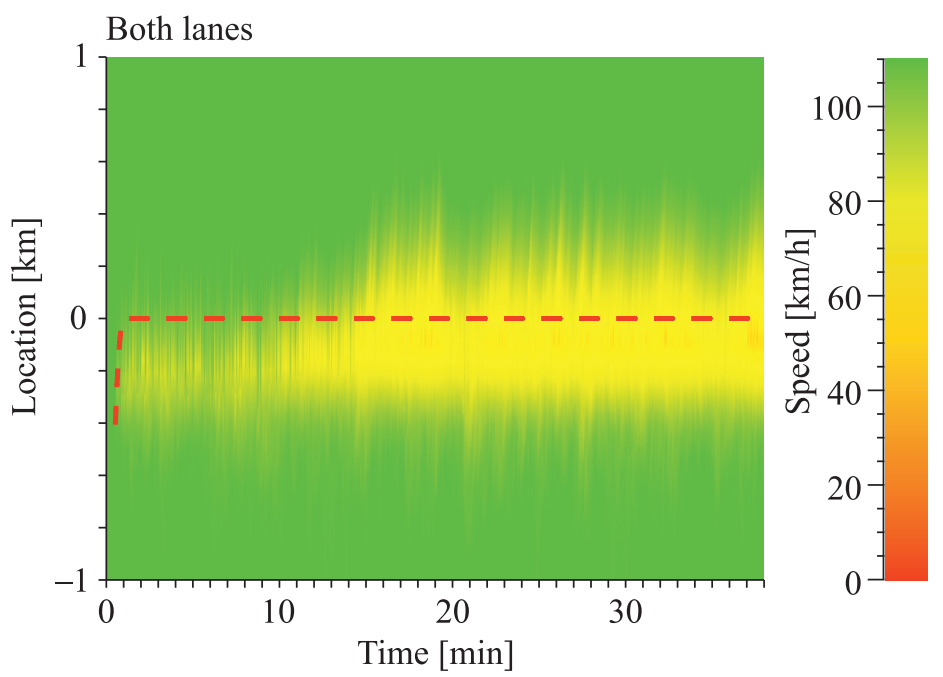

Figure 19. Another representation of Fig. 18. Two-dimensional representation of the simulation result as colorresolved speed (averaged over both lanes) versus road location and time

\section{Detection of a Stopped Vehicle (SV)}

\section{$S_{F}$ Phase Transition Points}

After the LSP has occurred due to an $\mathrm{F} \rightarrow \mathrm{S}$ transition (traffic breakdown) at the SV bottleneck, FCD vehicles register both $\mathrm{F}_{\mathrm{S}}$ phase transition points (which appear as a brown triangle standing on top in Fig. 21) and $S_{\mathrm{F}}$ phase transition points (which were marked as an upright dark green triangle). As in the scenario of $\mathrm{MB}, \mathrm{S}_{\mathrm{F}}$ phase transition points registered by the FCD vehicles are used to recognize a vehicle that has stopped.

We study below the time interval $0 \leq t<\mathrm{T}_{\mathrm{GP}}$. This is because, as we have found, the transformation of the LSP into the GP (the time interval $t \geq \mathrm{T}_{\mathrm{GP}}$ in Fig. 16) gives us almost no improvement in the quality of the SV detection.

The $S_{F}$ phase transition points shown in Fig. 21 correspond to $S_{F}$ phase transition points related to all vehicles driving through the SV bottleneck. Since we are only using the FCD vehicles that only have a small percentage $\gamma$ of all vehicles, we should recognize the SV through the use of $\mathrm{S}_{\mathrm{F}}$ phase transition points recognized by FCD vehicles only. In Fig. 22, there are three different realizations (from $M=1000$ different realizations), which we will use later in the statistical analysis, calculated at the penetrate rate $\gamma=2 \%$ of FCD vehicles. 

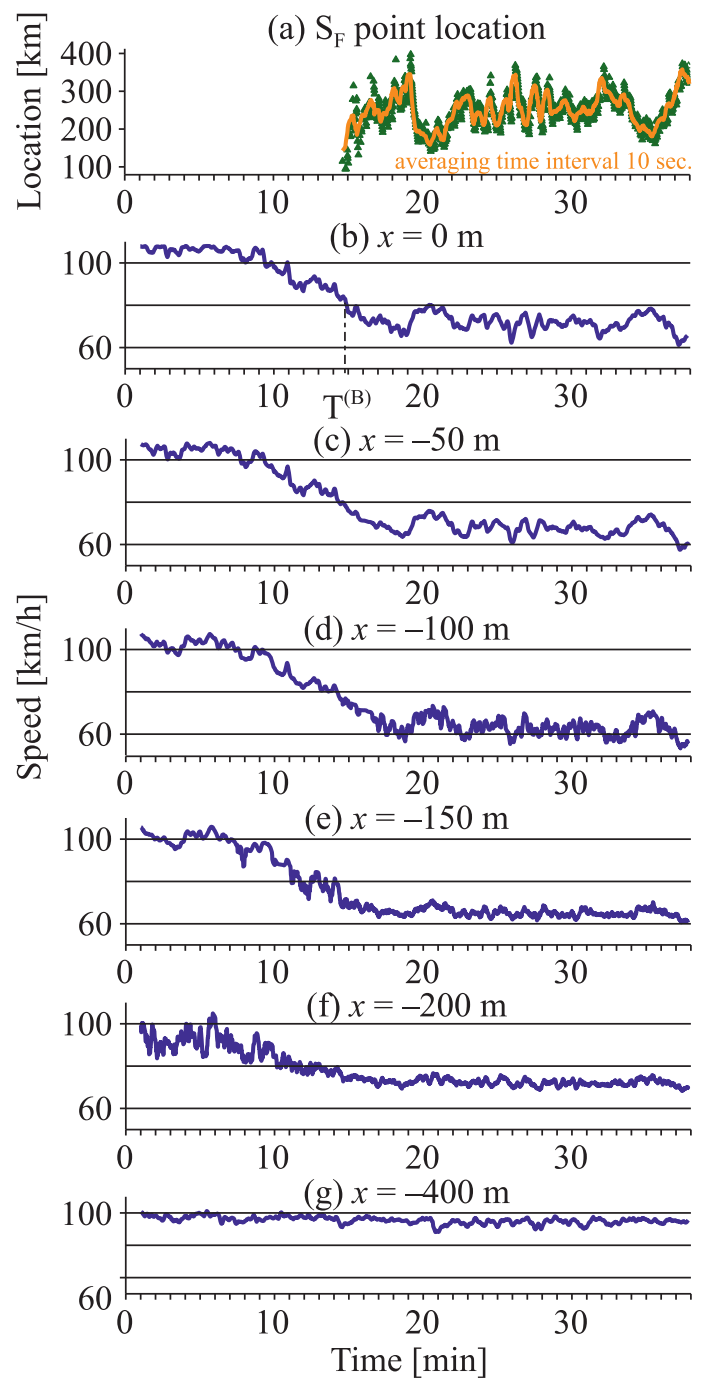

Figure 20. Locations of the $\mathrm{S}_{\mathrm{F}}$ phase transition points as a function of time (a) and the vehicle speed as a function of time at different road locations $(\mathrm{b}-\mathrm{g})$. The figures correspond to the time interval $0 \leq t<\mathrm{T}_{\mathrm{GP}}$, which is also used in Figs. 18 and 19

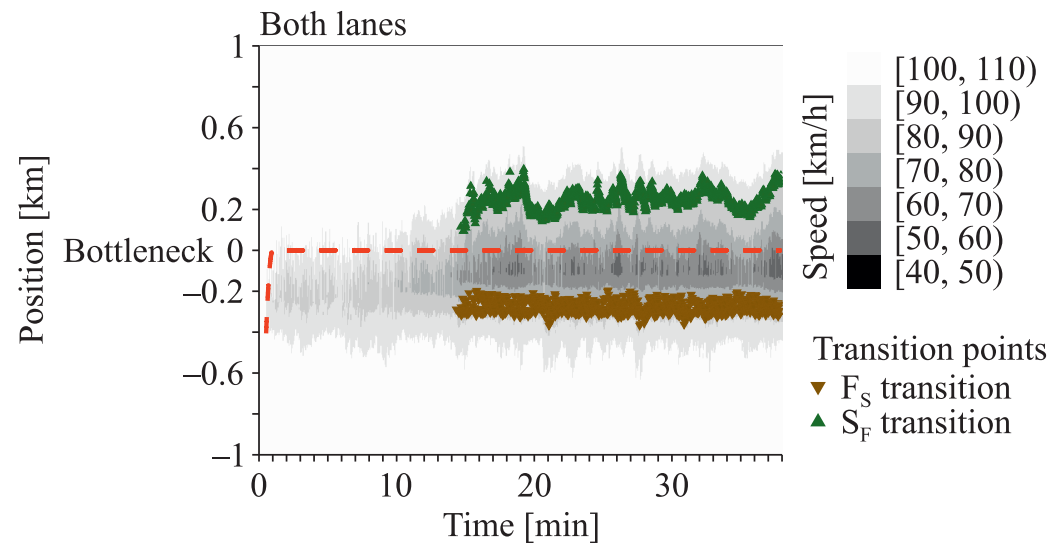

Figure 21. Two-dimensional representation of simulation results as gray shaded (averaged over both lanes) speed versus road location and time. The determined phase transition points are shown in color: $\mathrm{F}_{\mathrm{S}}$ as a brown triangle and $\mathrm{S}_{\mathrm{F}}$ as dark green triangle in the time interval $0 \leq t<\mathrm{T}_{\mathrm{GP}}$, which has also been used in Figs. 18 and 19 
(a) Realization 1

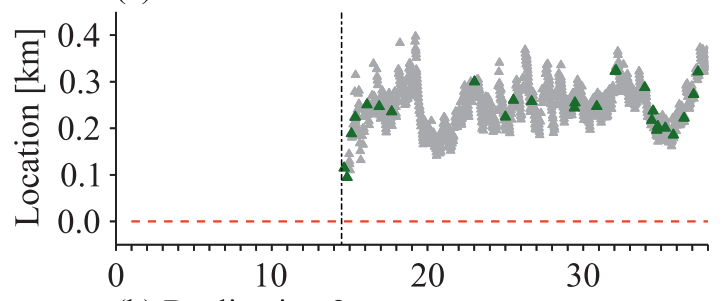

(b) Realization 2

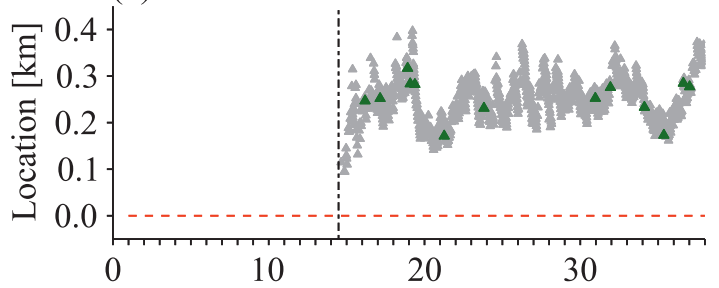

(c) Realization 3

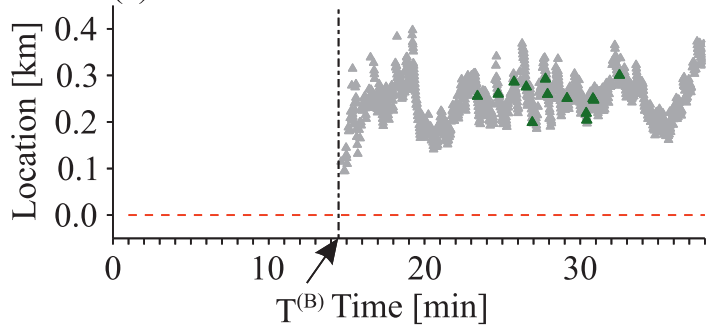

Figure 22. $\mathrm{S}_{\mathrm{F}}$ phase transition points (green triangles), which are recognized by $\gamma=2 \%$ FSD vehicles. Three different realizations of FCD vehicles in traffic: (a) realization 1, (b) realization 2, (c) realization 3. Gray triangles are $S_{\mathrm{F}}$ phase transition points that have been recognized by all vehicles in traffic

\section{Critical Number $K_{\mathrm{SV}}$ of $\mathrm{S}_{\mathrm{F}}$ Phase Transition Points and Corresponding Critical Time $t_{\mathrm{SV}}$ of Detection of $S V$ Bottleneck}

From Fig. 22 we can see that in different simulation realizations completely different $S_{\mathrm{F}}$ phase transition points have been recognized. Therefore, the critical number $K_{\mathrm{SV}}$ of $\mathrm{S}_{\mathrm{F}}$ phase transition points, which is necessary for the detection of the SV, is very different for different simulation realizations. Each critical number $K_{\mathrm{SV}}$ corresponds to some critical time $t_{\mathrm{SV}}$ at which the SV can be recognized. Thus, the recognition of the SV bottleneck occurs at different time instants $t_{\mathrm{SV}}$ for different simulation realizations. An example is shown in Figs. 23, 24 and 25 for simulation realizations 2 and 3.

We have the following characteristics of the critical number of $K_{\mathrm{SV}}$ of $\mathrm{S}_{\mathrm{F}}$ phase transition points and the corresponding critical time $t_{\mathrm{SV}}$ at which the detection of the SV is possible:

(i) After a few $S_{F}$ phase transition points have been recognized, condition (6) for the recognition of the SV is satisfied. Nevertheless, the critical number of $S_{\mathrm{F}}$ phase transition points is still not achieved: $K<K_{\mathrm{SV}}$. Only later, condition $K=K_{\mathrm{SV}}=7$ is satisfied (Fig. 23a).

(ii) If we increase the confidence level $p_{\alpha}$ from $p_{\alpha}=0.9$ in Fig. 23 (a) to $p_{\alpha}=0.99$, as expected, the critical number of $\mathrm{S}_{\mathrm{F}}$ phase transition points and the corresponding critical time $t_{\mathrm{SV}}$ at which the SV bottleneck is detected become larger than they have been in Fig. 23 (a): In Fig. 23 (b), $K=K_{\mathrm{SV}}=8$ and the corresponding critical time $t_{\mathrm{SV}}=31 \mathrm{~min}$ (Fig. 23b).

(iii) Another characteristic of the method for predicting the SV bottleneck is as follows. We have simulated the prediction of the SV bottleneck for different parameter $\Delta_{\mathrm{SV}}$ in conditions (6). We have found that at the same chosen confidence level $p_{\alpha}=0.9$ very different values of the critical number $K=K_{\mathrm{SV}}$ of the $S_{\mathrm{F}}$ phase transition points and the corresponding critical times $t_{\mathrm{SV}}$ can be reached (Fig. 24) by choosing the different selected parameter $\Delta_{\mathrm{SV}}$ : The larger the chosen parameter $\Delta_{\mathrm{SV}}$, 
(a) Realization $2, p_{\alpha}=0.9, \Delta_{\mathrm{SV}}=2 \mathrm{~km} / \mathrm{h}$

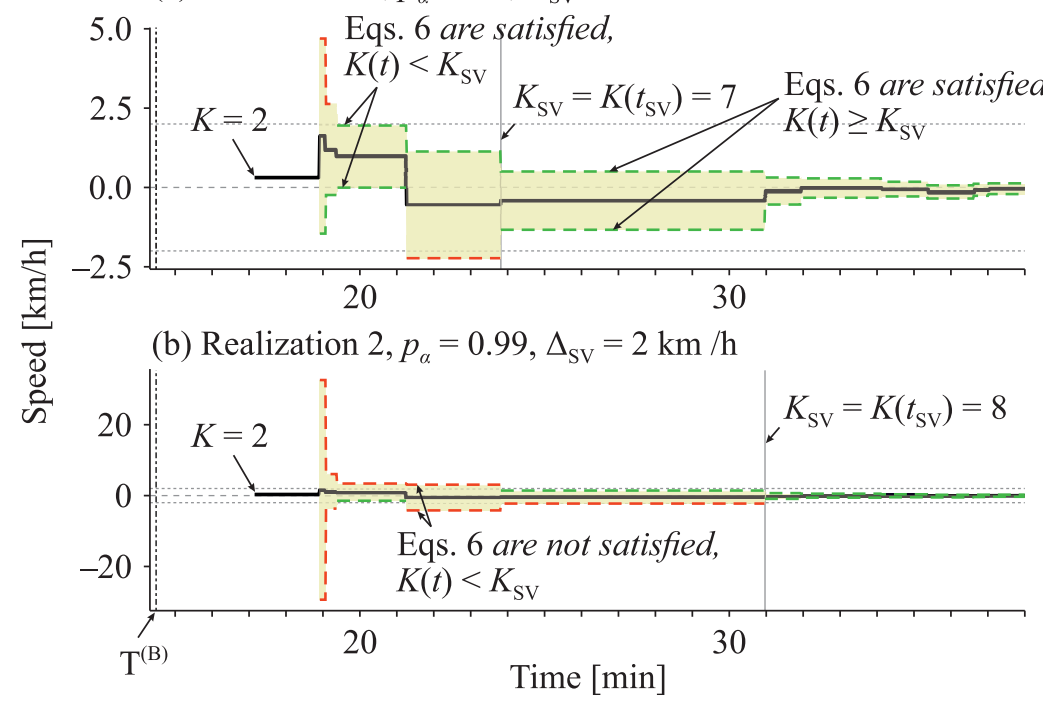

Figure 23. Dependence of the critical number $K_{\mathrm{SV}}$ of the $\mathrm{S}_{\mathrm{F}}$ phase transition points and the corresponding critical time $t_{\mathrm{SV}}$ at which the detection of the $\mathrm{SV}$ is possible on the chosen parameter $p_{\alpha}$ for realization 2 in Fig. 22. $\Delta_{\mathrm{SV}}=2 \mathrm{~km} / \mathrm{h}$ : (a) $p_{\alpha}=0.9$. (b) $p_{\alpha}=0.99$

(a) Realization $2, p_{\alpha}=0.9, \Delta_{\mathrm{sv}}=1 \mathrm{~km} / \mathrm{h}$
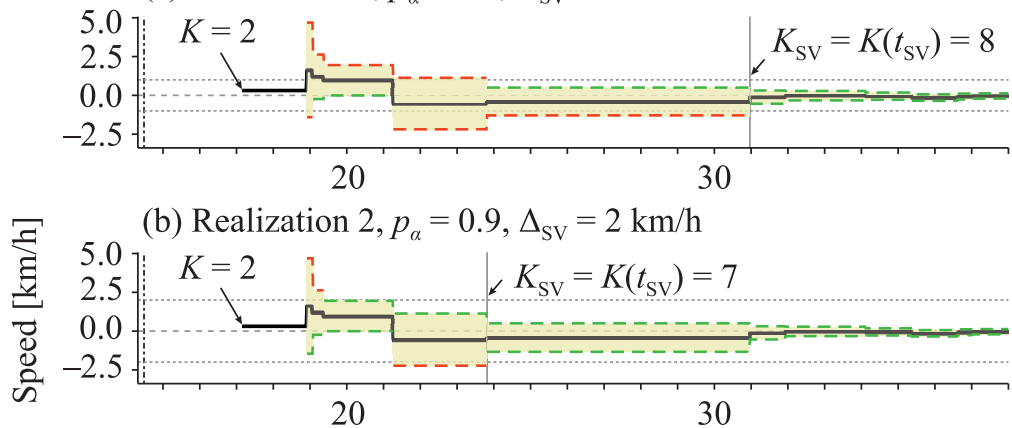

(c) Realization 2, $p_{\alpha}=0.9, \Delta_{\mathrm{SV}}=3 \mathrm{~km} / \mathrm{h}$

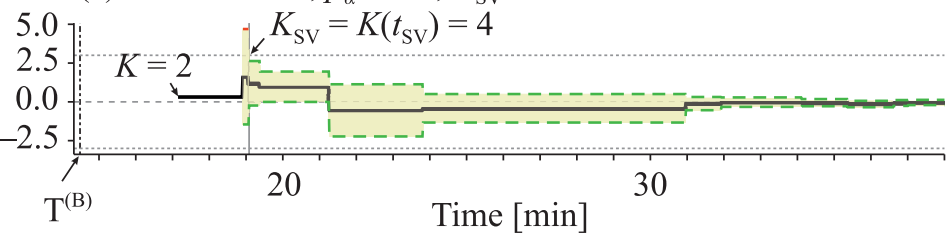

Figure 24. Dependence of the critical number $K_{\mathrm{SV}}$ of the $\mathrm{S}_{\mathrm{F}}$ phase transition points and the corresponding critical time $t_{\mathrm{SV}}$ at which the detection of the SV is possible on the chosen parameter $\Delta_{\mathrm{SV}}$ for realization 2 in Fig. $22 \mathrm{~b}$. $p_{\alpha}=0.9:$ (a) $\Delta_{\mathrm{Sv}}=1 \mathrm{~km} / \mathrm{h}$, (b) $\Delta_{\mathrm{Sv}}=2 \mathrm{~km} / \mathrm{h}$, (c) $\Delta_{\mathrm{Sv}}=3 \mathrm{~km} / \mathrm{h}$

the smaller the critical number $K=K_{\mathrm{SV}}$ of $\mathrm{S}_{\mathrm{F}}$ phase transition points and the corresponding critical time $t_{\mathrm{SV}}$. However, there is a limitation to this property: If for the example in Fig. 24 we assume $\Delta_{\mathrm{SV}}>3 \mathrm{~km} / \mathrm{h}$, we have found no change in the values of the critical number $K=K_{\mathrm{SV}}$ of $\mathrm{S}_{\mathrm{F}}$ phase transition points and the corresponding critical time $t_{\mathrm{SV}}$. We have found the same behavior in other simulation realizations (Fig. 25). 
(a) Realization 3, $p_{\alpha}=0.9, \Delta_{\mathrm{SV}}=1 \mathrm{~km} / \mathrm{h}$

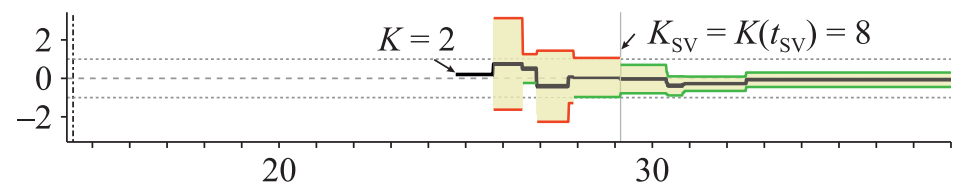

(b) Realization 3, $p_{\alpha}=0.9, \Delta_{\mathrm{SV}}=2 \mathrm{~km} / \mathrm{h}$

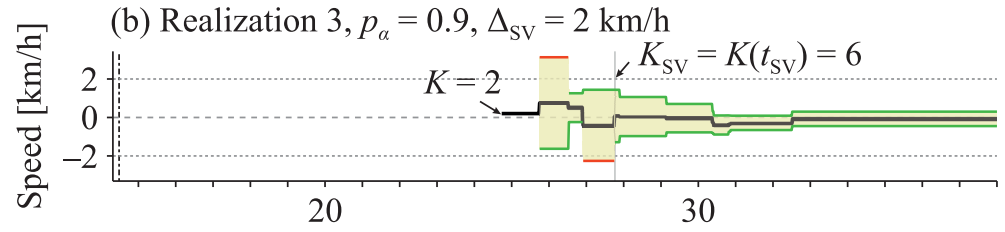

(c) Realization 3, $p_{\alpha}=0.9, \Delta_{\mathrm{SV}}=3 \mathrm{~km} / \mathrm{h}$

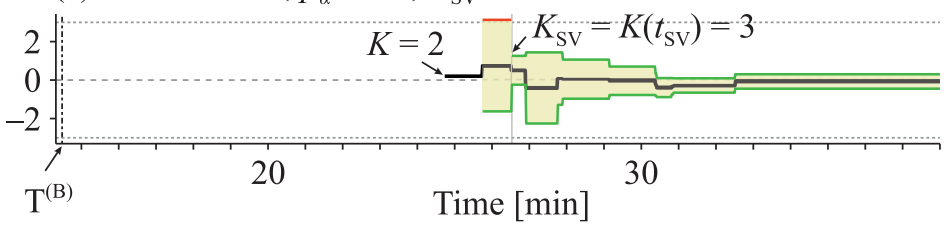

Figure 25. Dependence of the critical number $K_{\mathrm{SV}}$ of the $\mathrm{S}_{\mathrm{F}}$ phase transition points and the corresponding critical time $t_{\mathrm{SV}}$ at which the detection of the $\mathrm{SV}$ is possible on the chosen parameter $\Delta_{\mathrm{SV}}$ for realization 3 in Fig. $22 \mathrm{c}$. $p_{\alpha}=0.9:$ (a) $\Delta_{\mathrm{SV}}=1 \mathrm{~km} / \mathrm{h}$, (b) $\Delta_{\mathrm{SV}}=2 \mathrm{~km} / \mathrm{h}$, (c) $\Delta_{\mathrm{SV}}=3 \mathrm{~km} / \mathrm{h}$

\section{Statistical Analysis of Prediction of an SV Bottleneck}

In the previous section we examined the characteristics of the prediction of the SV bottleneck. There we have only used one possible realization, which happened in real traffic. Here, we do statistical analysis of the prediction of the SV bottleneck. In this statistical analysis, we used $M=1000$ different random realizations to determine the quality the prediction of the SV bottleneck. After that, in the next section, the quality of the prediction of the SV bottleneck and the quality the prediction of a moving bottleneck (MB) are compared.

\section{Statistics of the Critical Number $K_{S V}$ of $S_{F}$ Phase Transition Points and Critical Time $t_{S V}$ for SV Prediction}

We have seen that a critical number $K_{\mathrm{SV}}$ of $\mathrm{S}_{\mathrm{F}}$ phase transition points registered by FCD vehicles in a simulation realization is necessary to achieve to predict an SV at a selected confidence level $p_{\alpha}$. In different simulation realizations of the FCD vehicles, the critical number $K_{\mathrm{SV}}$ of $\mathrm{S}_{\mathrm{F}}$ phase transition points can have very different values. One of the reasons is a very different time instant at which the first FCD vehicle has registered $S_{F}$ phase transition point in different simulation realizations as well as different random distributions of FCD vehicles in the realizations. Therefore, it is interesting to examine how the critical number $K_{\mathrm{SV}}$ of $\mathrm{S}_{\mathrm{F}}$ phase transition points are statistically distributed in different realizations and how this distribution varies depending on the method parameters for the prediction of the SV (Figs. 26 and 27).

We have obtained the following results:

(i) If the confidence level $p_{\alpha}$ is chosen and the FCD rate $\gamma$ changes, we have found that the larger the FCD rate $\gamma$, the larger the spread of the distribution of the critical number $K_{\mathrm{SV}}$ of $\mathrm{S}_{\mathrm{F}}$ phase transition points is (Figs. 26 and 27).

(ii) If the confidence level $p_{\alpha}$ is chosen and the FCD rate $\gamma$ changes, we have found that, the larger the $\mathrm{FCD}$ rate $\gamma$, the larger the mean of the distribution of the critical number $K_{\mathrm{SV}}$ of $\mathrm{S}_{\mathrm{F}}$ phase transition points (Figs. 26 and 27). 

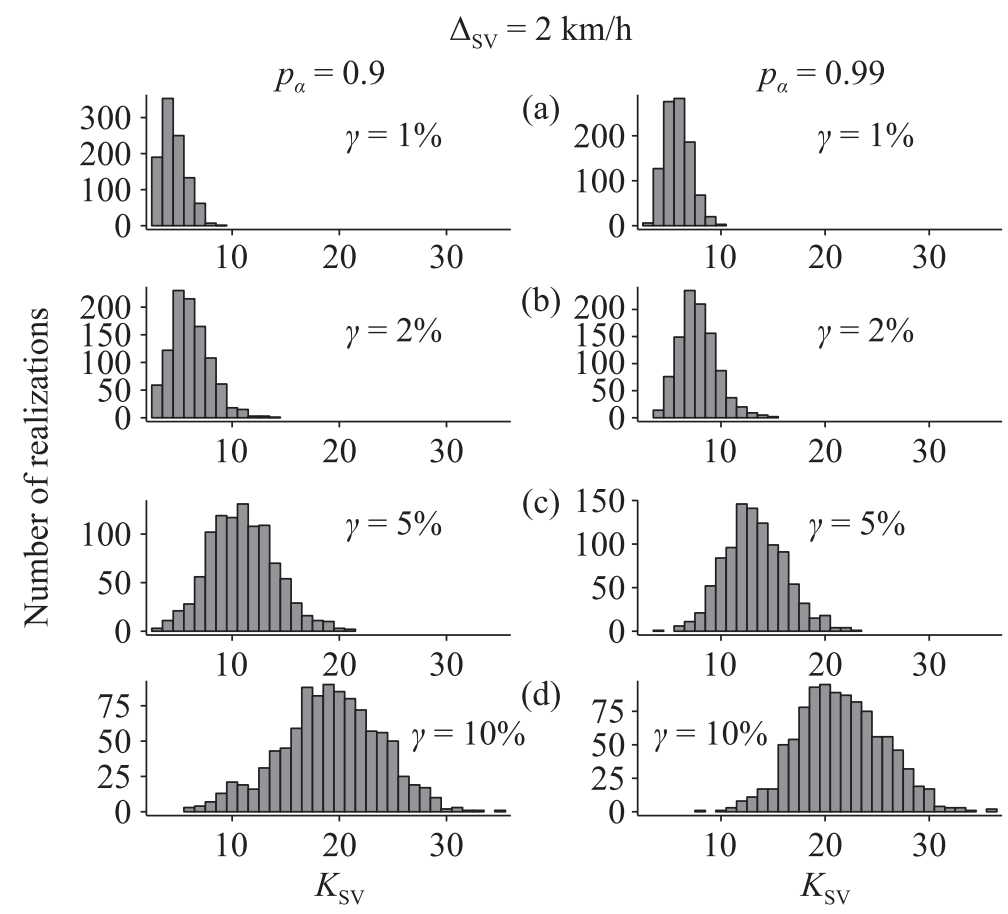

Figure 26. Statistical analysis of the distribution of the critical number $K_{\mathrm{SV}}$ of $\mathrm{S}_{\mathrm{F}}$ phase transition points, which is necessary for the prediction of the SV, for all $M=1000$ realizations that have been used. $\Delta_{\mathrm{SV}}=2 \mathrm{~km} / \mathrm{h}$. For the figures in the left column, the selected confidence level $p_{\alpha}=0.9$. For the figures in the right column, the selected confidence level $p_{\alpha}=0.99$ : (a) $\gamma=1 \%$, (b) $\gamma=2 \%$, (c) $\gamma=5 \%$, (d) $\gamma=10 \%$

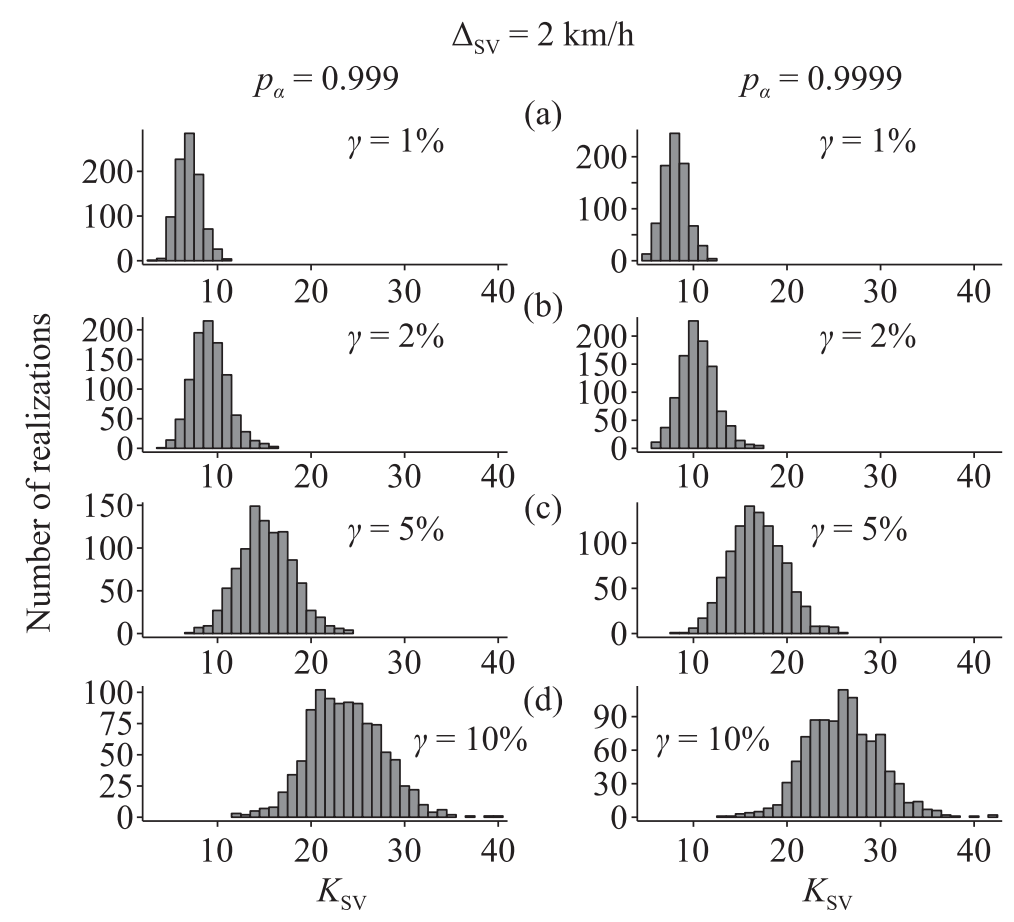

Figure 27. Statistical analysis of the distribution the critical number of $K_{\mathrm{SV}}$ of $\mathrm{S}_{\mathrm{F}}$ phase transition points, which is necessary for the prediction of the SV, for all $M=1000$ realizations that have been used. $\Delta_{\mathrm{SV}}=2 \mathrm{~km} / \mathrm{h}$. For the figures in the left column, the selected confidence level $p_{\alpha}=0.999$. For the figures in the right column, the selected confidence level $p_{\alpha}=0.9999$ : (a) $\gamma=1 \%$, (b) $\gamma=2 \%$, (c) $\gamma=5 \%$, (d) $\gamma=10 \%$ 
(iii) If the confidence level $p_{\alpha}$ is chosen and the FCD rate $\gamma$ changes, we have found that, the larger the FCD rate $\gamma$, the larger the minimum value of the critical number $K_{\mathrm{SV}}$ of $\mathrm{S}_{\mathrm{F}}$ phase transition points (Figs. 26 and 27).

(iv) The larger the level of confidence $p_{\alpha}$ is chosen (Figs. 26 and 27), the larger these changes in the distribution of the critical number $K_{\mathrm{SV}}$ of $\mathrm{S}_{\mathrm{F}}$ phase transition points as a function of the FCD rate $\gamma$.

To understand these results, we turn to Figs. 28 and 29 made for further statistical analysis. In this analysis, the distributions of the critical time $t_{\mathrm{SV}}$, which is necessary for the prediction of the $\mathrm{SV}$, as a function of the FCD vehicle rate $\gamma$ have been calculated.

(i) We have found that the larger the FCD rate $\gamma$, the narrower the distribution of the critical time $t_{\mathrm{SV}}$ is (Figs. 28 and 29). This result is the opposite of the above result that the larger the FCD rate $\gamma$, the more spread the distribution of the critical number $K_{\mathrm{SV}}$ of $\mathrm{S}_{\mathrm{F}}$ of phase transition points is (Figs. 26 and 27).

(ii) We have found that, the larger the FCD rate $\gamma$ (Figs. 28, 29 and 30), the smaller the mean of the distribution of the critical time $t_{\mathrm{SV}}^{(\mathrm{mean})}$. This result is the opposite the above result that, the larger the FCD rate $\gamma$ (Figs. 26 and 27), the larger the mean of the distribution of the critical number $K_{\mathrm{Sv}}$ of $\mathrm{S}_{\mathrm{F}}$ phase transition points.

(iii) We have found that the minimum of the critical time $t_{\mathrm{SV}}^{(\mathrm{min})}$ is a complex non-monotonic function the FCD rate is $\gamma$ (Fig. 31). The critical time $t_{\mathrm{SV}}^{(\mathrm{min})}$ changes as a function of the FCD rate $\gamma$ greatly if another confidence level $p_{\alpha}$ and / or another parameter $\Delta_{\mathrm{SV}}$ is chosen. For example, the critical time $t_{\mathrm{SV}}^{(\mathrm{min})}$ with small values of the FCD rate $\gamma$ increases as the FCD rate $\gamma$ increases (curves 3 and 4 in Fig. $31(a, b))$.

This complexity of the critical time $t_{\mathrm{SV}}^{(\mathrm{min})}$ as a function of the FCD rate $\gamma$ (Fig. 31) contradicts the above result, that, the larger the FCD rate $\gamma$ is (Figs. 26 and 27), the larger the minimum of the value of the critical number $K_{\mathrm{SV}}$ of $\mathrm{S}_{\mathrm{F}}$ phase transition points.

To understand these contradictions between the functions of the critical number $K_{\mathrm{SV}}$ of $\mathrm{S}_{\mathrm{F}}$ phase transition points and the associated critical time $t_{\mathrm{SV}}$ from the FCD rate $\gamma$, we have calculated the critical number $K_{\mathrm{SV}}$ of $\mathrm{S}_{\mathrm{F}}$ phase transition points and the associated critical time $t_{\mathrm{SV}}$ for two different simulation realizations of the FCD vehicles that correspond to two very different FCD rates $\gamma$ (Fig. 33). We have the following results:

(i) If the number $K$ of $\mathrm{S}_{\mathrm{F}}$ phase transition points is relatively small, the error term $t_{\alpha, K-2}^{(2)} \sigma_{\mathrm{rec}}(K)$ in (6) depends strongly on the FCD rate $\gamma$ : For a given value $K$, it has been found that, the larger the FCD rate $\gamma$, the larger the error term $t_{\alpha, K-2}^{(2)} \sigma_{\text {rec }}(K)$ in (6) is (Fig. 32).

This result is due to the decrease in the mean time interval between two FCD vehicles following one another in simulation realizations under increasing the FCD rate $\gamma$. Thereby the fluctuations between road locations related to two following one another $\mathrm{S}_{\mathrm{F}}$ phase transition points lead to a larger mean error in the speed $v_{\text {rec }}$ than the mean error occurring at a smaller FCD rate $\gamma$.

(ii) The last result for the relatively small FCD rate $\gamma=2 \%$ is shown in Fig. 33a and for a large FCD rate $\gamma=10 \%$ is illustrated in Fig. 33b: For small values $K$ the values of the error term $t_{\alpha, K-2}^{(2)} \sigma_{\text {rec }}(K)$ in (6) are much smaller for the relatively small FCD rate $\gamma=2 \%$ (Fig. 33a) than the values of the error term are for the larger FCD rate $\gamma=10 \%$ (Fig. 33b). Therefore, the critical number $K_{\mathrm{SV}}$ of the $\mathrm{S}_{\mathrm{F}}$ phase transition points with the large FCD rate $\gamma=10 \%$ is much larger (Fig. 33b) than the critical number $K_{\mathrm{SV}}$ of $\mathrm{S}_{\mathrm{F}}$ phase transition points is at a lower FCD rate $\gamma=2 \%$ (Fig. 33a). In contrast, the critical time $t_{\mathrm{SV}}$ at large FCD rate $\gamma=10 \%$ is much smaller (Fig. 33b) than the critical time $t_{\mathrm{SV}}$ is at a lower FCD rate $\gamma=2 \%$ (Fig. 33a). 

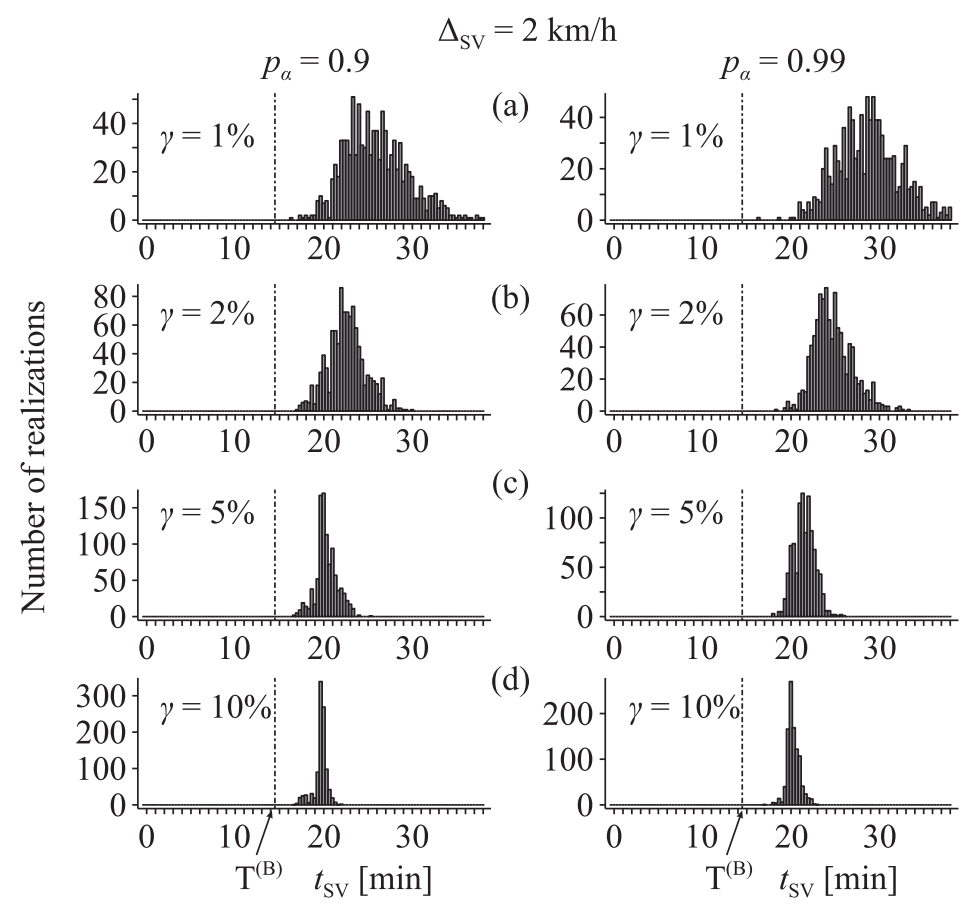

bin width $=20 \mathrm{sec}$.

Figure 28. Statistical analysis of the distribution of the critical time $t_{\mathrm{SV}}$, which is necessary for the prediction of the SV, as a function of the FCD vehicle rate. All $M=1000$ realizations of the statistical analysis were used: $\Delta_{\mathrm{SV}}=2 \mathrm{~km} / \mathrm{h}$. (a) $\gamma=1 \%$, (b) $\gamma=2 \%$, (c) $\gamma=5 \%$, (d) $\gamma=10 \%$. For the figures in the left column, the chosen confidence level $p_{\alpha}=0.9$. For the figures in the right column, the chosen confidence level $p_{\alpha}=0.99$
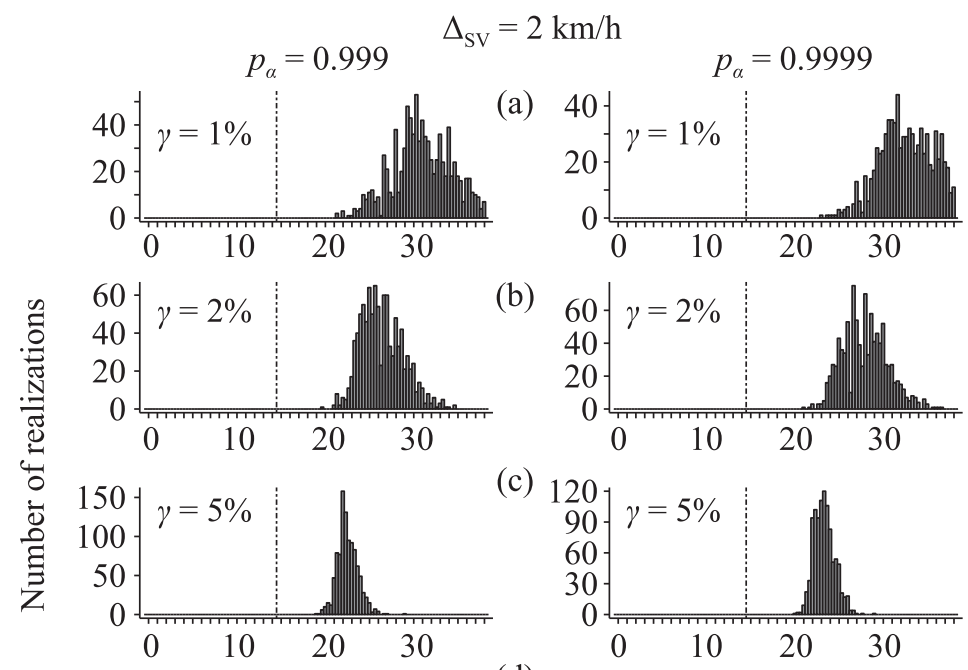

(c) 120
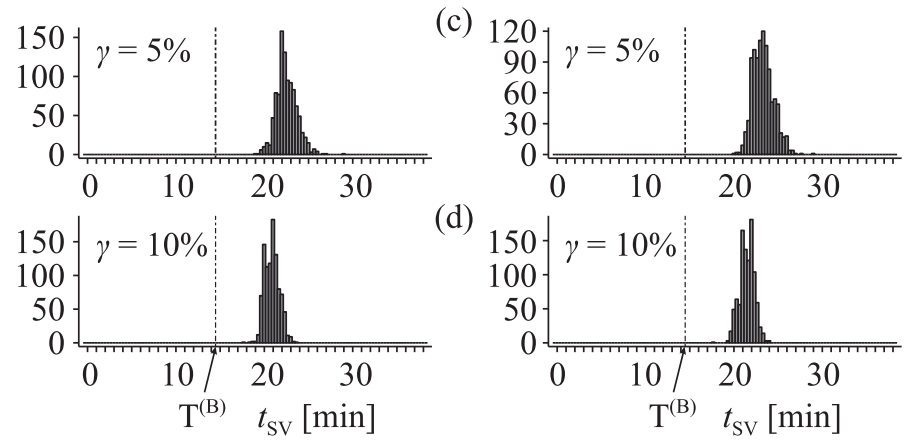

bin width $=20 \mathrm{sec}$.

Figure 29. Statistical analysis of the distribution of the critical time $t_{\mathrm{SV}}$, which is necessary for the detection of the SV, on the FCD vehicle rate. All $M=1000$ realizations of the statistical analysis were used: $\Delta_{\mathrm{SV}}=2 \mathrm{~km} / \mathrm{h}$. (a) $\gamma=1 \%$, (b) $\gamma=2 \%$, (c) $\gamma=5 \%$, (d) $\gamma=10 \%$. For the figures in the left column, the chosen confidence level $p_{\alpha}=0.999$. For the figures in the right column, the chosen confidence level $p_{\alpha}=0.9999$ 
(a) $\Delta_{\mathrm{SV}}=1 \mathrm{~km} / \mathrm{h}$
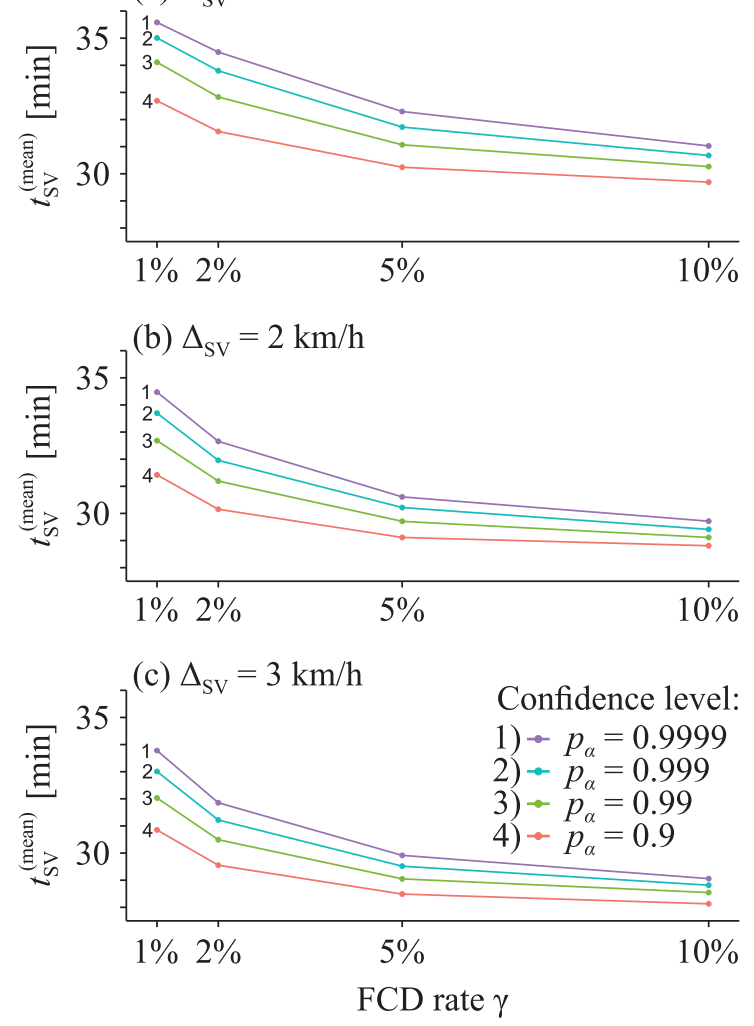

Figure 30. Statistical analysis of the mean value of the critical time $t_{\mathrm{SV}}^{\text {(mean) }}$ for the prediction of the SV as a function of the FCD vehicle rate $\gamma$ : (a) $\Delta_{\mathrm{SV}}=1 \mathrm{~km} / \mathrm{h}$. (b) $\Delta_{\mathrm{SV}}=2 \mathrm{~km} / \mathrm{h}$. (c) $\Delta_{\mathrm{Sv}}=3 \mathrm{~km} / \mathrm{h}$. Curves 1)-5) are related to different chosen confidence level: $p_{\alpha}=0.9999$ (curve 1) $p_{\alpha}=0.999$ (curve 2), $p_{\alpha}=0.99$ (curve 3), $p_{\alpha}=0.9$ (curve 4)
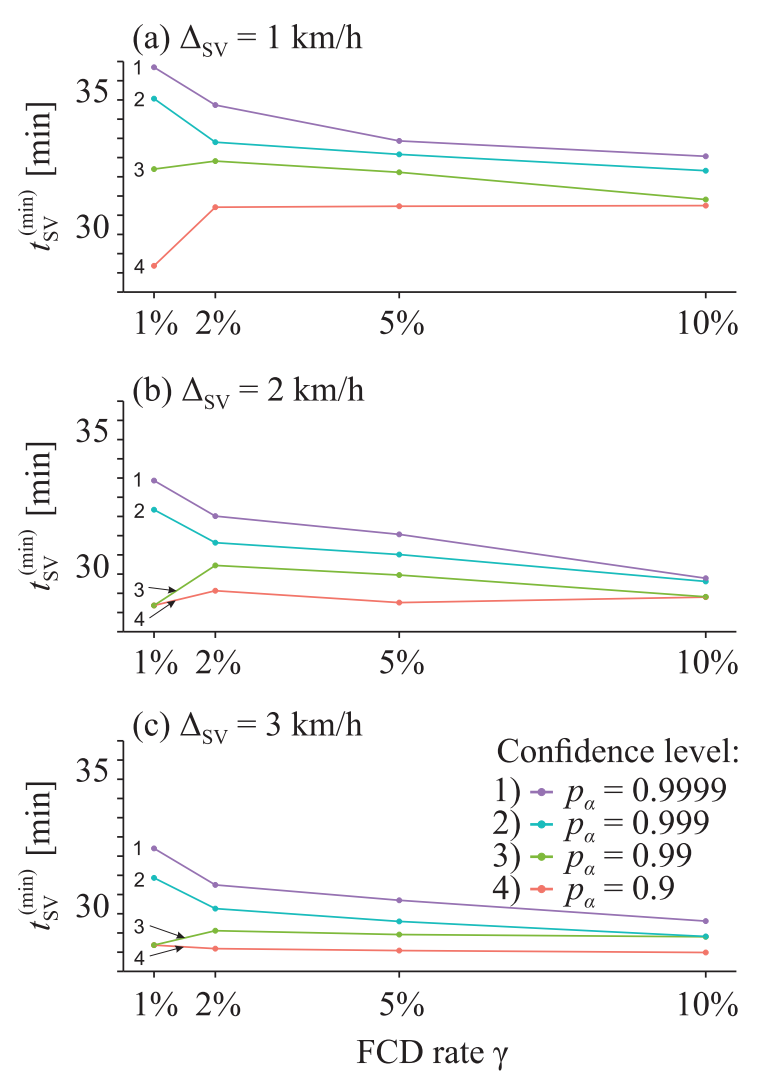

Figure 31. Statistical analysis of the minimum number of critical time $t_{\mathrm{SV}}^{(\mathrm{min})}$ for the detection of the $\mathrm{SV}$, which has been found in $M=1000$ realizations, as a function of the FCD vehicle rate $\gamma$ : (a) $\Delta_{\mathrm{SV}}=1 \mathrm{~km} / \mathrm{h}$. (b) $\Delta_{\mathrm{SV}}=2 \mathrm{~km} / \mathrm{h}$. (c) $\Delta_{\mathrm{SV}}=3 \mathrm{~km} / \mathrm{h}$. Curves 1)-5) are related to different chosen confidence level: $p_{\alpha}=0.9999$ (curve 1) $p_{\alpha}=0.999$ (curve 2), $p_{\alpha}=0.99$ (curve 3), $p_{\alpha}=0.9$ (curve 4$)$

FCD Rate: $\gamma=2 \& 10 \%, p_{\alpha}=0.9$

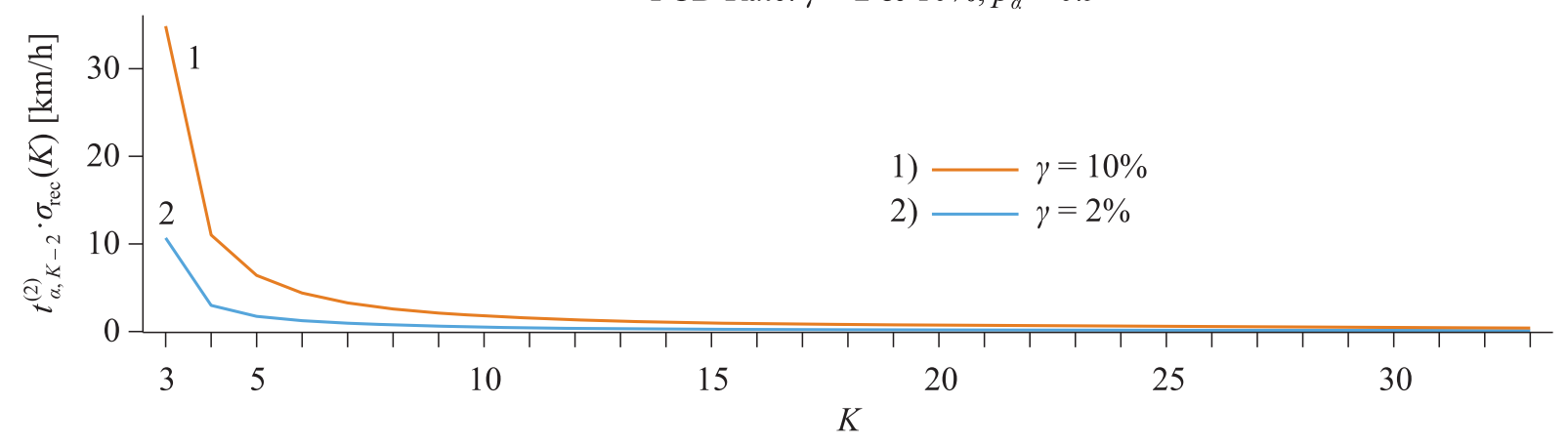

Figure 32. Statistical analysis of the mean value of the error term $t_{\alpha, K-2}^{(2)} \sigma_{\text {rec }}(K)$ in (6) as a function of the FCD vehicle rate $\gamma \cdot p_{\alpha}=0.9$ 
(a) Realization 3 with $\gamma=2 \%, p_{\alpha}=0.9, \Delta_{\mathrm{SV}}=2 \mathrm{~km} / \mathrm{h}$

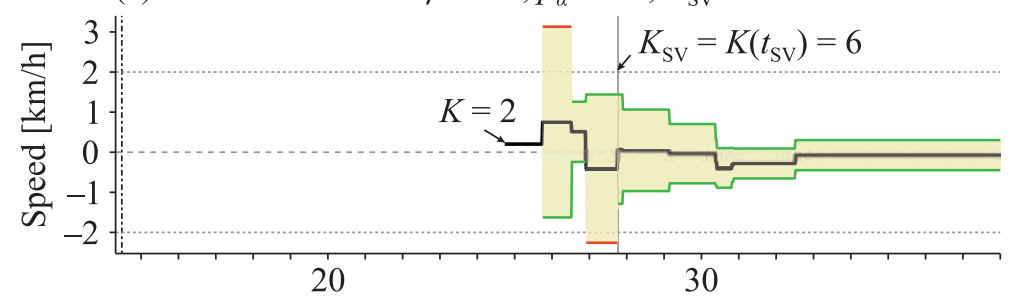

(b) A realization with $\gamma=10 \%, p_{\alpha}=0.9, \Delta_{\mathrm{SV}}=2 \mathrm{~km} / \mathrm{h}$

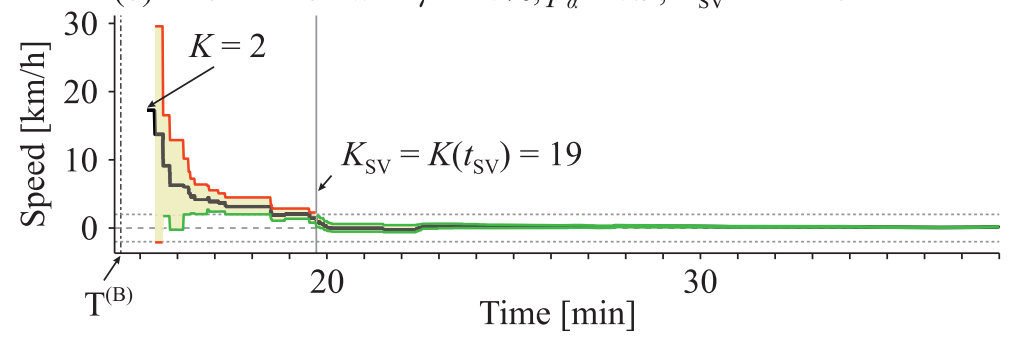

Figure 33. Dependence of the critical number $K_{\mathrm{SV}}$ of $\mathrm{S}_{\mathrm{F}}$ phase transition points and the corresponding critical time $t_{\mathrm{SV}}$, which is necessary for the prediction of the SV, on the FCD vehicle rate: (a) Realization 3 for $\gamma=2 \%$ taken from Fig. 22. (b) One of the realizations for $\gamma=10 \% . \Delta_{\mathrm{SV}}=2 \mathrm{~km} / \mathrm{h}, p_{\alpha}=0.9$

(a) FCD rate: $\gamma=1 \%, \Delta_{\mathrm{SV}}=2 \mathrm{~km} / \mathrm{h}$

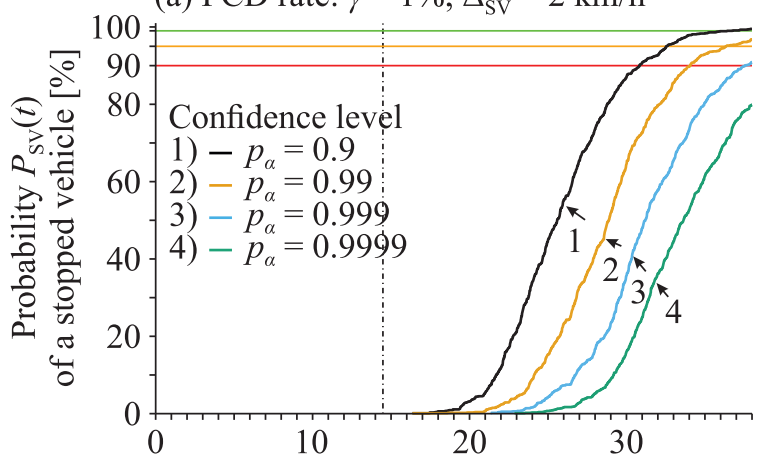

(b) FCD rate: $\gamma=2 \%, \Delta_{\mathrm{SV}}=2 \mathrm{~km} / \mathrm{h}$

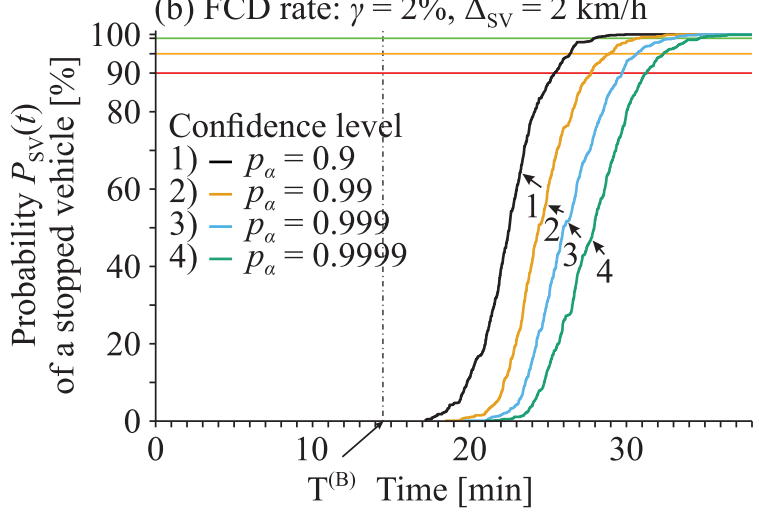

Figure 34. Probabilities of the prediction of the SV for the chosen parameter $\Delta_{\mathrm{SV}}=2 \mathrm{~km} / \mathrm{h}$ : (a) FCD rate $\gamma=1 \%$. (b) FCD rate $\gamma=2 \%$. Four curves 1)-4) correspond to the confidence level $p_{\alpha}=0.9$ to 0.9999 (a) FCD rate: $\gamma=5 \%, \Delta_{\mathrm{SV}}=2 \mathrm{~km} / \mathrm{h}$

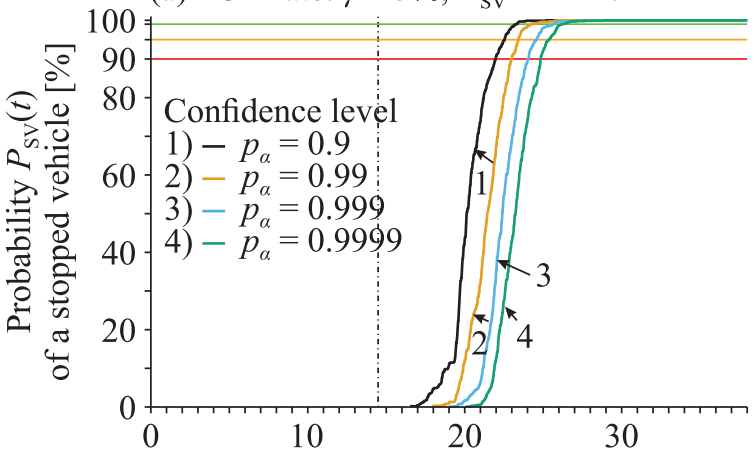

(b) FCD rate: $\gamma=10 \%, \Delta_{\mathrm{SV}}=2 \mathrm{~km} / \mathrm{h}$

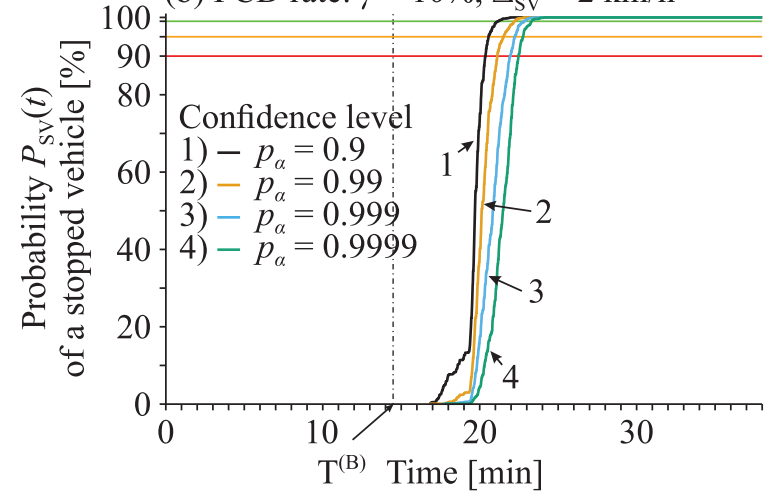

Figure 35. Probabilities of the prediction of the SV for the chosen parameter $\Delta_{\mathrm{SV}}=2 \mathrm{~km} / \mathrm{h}$ : (a) FCD rate $\gamma=5 \%$. (b) FCD rate $\gamma=10 \%$. Four curves 1)-4) correspond to the confidence level $p_{\alpha}=0.9$ to 0.9999 


\section{Probability of Recognition of SV Bottleneck}

The probability of prediction of the SV bottleneck has been calculated through the formula (10) (Figs. 34, 35 and 36).

We have the following results:

(i) A SV cannot be recognized by FCD vehicles in free traffic: The probability of the SV being recognized is zero as long as there is no traffic breakdown at the SV bottleneck (Figs. 34, 35 and 36). The physical explanation of this result is that the SV can only be recognized if the FCD vehicles register $\mathrm{S}_{\mathrm{F}}$ phase transition points. It is theoretically also possible that $\mathrm{S}_{\mathrm{F}}$ phase transition points are registered by FCD vehicles through the $\mathrm{F} \rightarrow \mathrm{S} \rightarrow \mathrm{F}$ phase transitions, which can happen before a traffic breakdown occurs at the SV bottleneck. However, in the simulated example of traffic flow during the time interval $0<t<T^{(\mathrm{B})}$ no $\mathrm{F} \rightarrow \mathrm{S} \rightarrow \mathrm{F}$ phase transitions occur.

(ii) There is a time delay between the time of traffic breakdown at the SV bottleneck $t=T^{(\mathrm{B})}$ and the time instant $t_{\mathrm{SV}}$ at which the SV has been recognized. This time delay $\Delta t_{\mathrm{SV}}=t_{\mathrm{SV}}-T^{(\mathrm{B})}$ corresponds to the value of the number of $K_{\mathrm{SV}}$ of $\mathrm{S}_{\mathrm{F}}$ phase phase transition points, which is necessary for the recognition of the SV in a simulation realization.

For statistical analysis, this means that there is a minimum point in time $t_{\mathrm{SV}}=t_{\mathrm{SV}}^{(\mathrm{min})}$ at which in at least one of the $M=1000$ realizations an SV has been detected by FCD vehicles. This minimum time $t_{\mathrm{SV}}^{(\mathrm{min})}$ corresponds to the minimum value of the critical number $K_{\mathrm{SV}}=K_{\mathrm{SV}}^{(\mathrm{min})}$ of $\mathrm{S}_{\mathrm{F}}$ phase transition points that are necessary to register by FCD vehicles to detect the SV in this realization. The minimum time $t_{\mathrm{SV}}^{(\mathrm{min})}$ is related to the first time at which the probability of the prediction of the SV $P_{\mathrm{SV}}(t)$ is larger than zero (Figs. 34, 35 and 36).
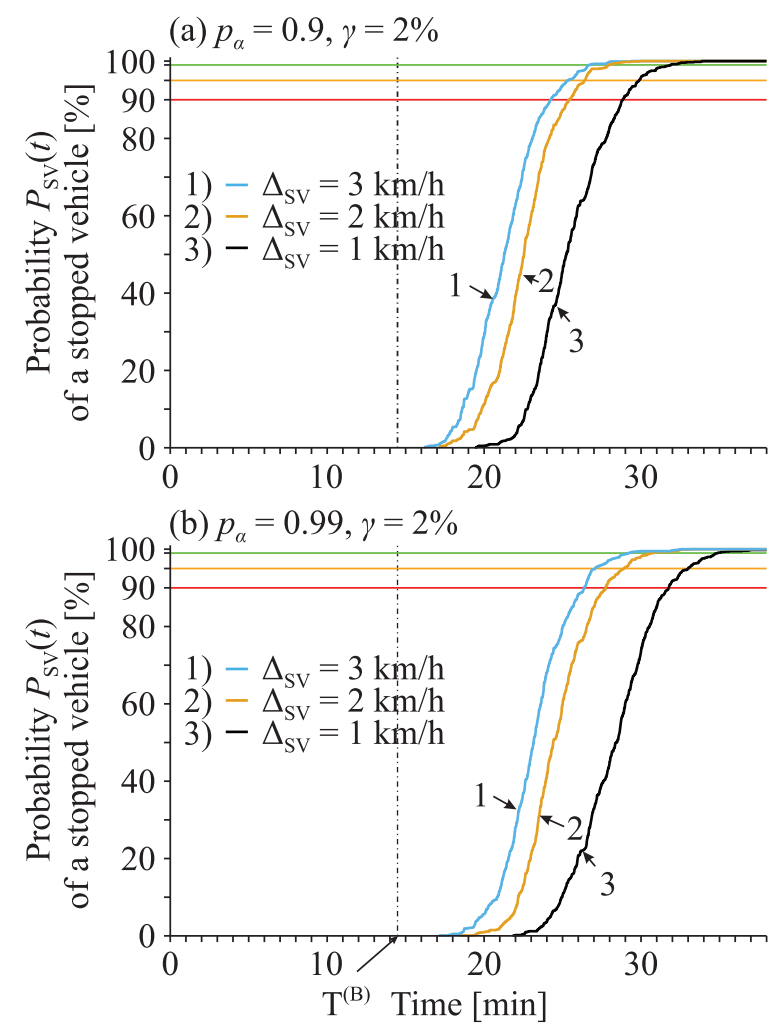

Figure 36. Probabilities of the prediction of the SV for FCD rate $\gamma=2 \%$ and for various parameters $\Delta_{\mathrm{SV}}=1$ $\mathrm{km} / \mathrm{h}$ for curve 1), $\Delta_{\mathrm{SV}}=2 \mathrm{~km} / \mathrm{h}$ for curve 2) and $\Delta_{\mathrm{SV}}=3 \mathrm{~km} / \mathrm{h}$ for curve 3). (a) Confidence level $p_{\alpha}=0.9$. (b) Confidence level $p_{\alpha}=0.99$ 
(iii) The minimum time $t_{\mathrm{SV}}=t_{\mathrm{SV}}^{(\mathrm{min})}$, at which in at least one of the $M=1000$ realizations an $\mathrm{SV}$ has been recognized by FCD vehicles, depends on the following parameters of the method used to detect the SV: the FCD rate $\gamma$, the selected parameter $\Delta_{\mathrm{SV}}$, and confidence level $p_{\alpha}$. The minimum time $t_{\mathrm{SV}}=t_{\mathrm{SV}}^{(\mathrm{min})}$ increases particularly strongly if the confidence level $p_{\alpha}$ is increased (Fig. 34). However, it only applies if the FCD rate $\gamma$ is small. When the FCD rate $\gamma$ increases, the difference between the minimum times $t_{\mathrm{SV}}=t_{\mathrm{SV}}^{(\mathrm{min})}$ related to the different confidence level $p_{\alpha}$ decreases (Fig. 35).

\section{Statistical Analysis of Location Prediction of SV}

After the LSP is transformed into the GP, additional phase transition points are registered by FCD vehicles (see $F_{S}, S_{F}, S_{J}$, and $J_{S}$ phase transition points in Fig. 37).

However, the positions of $F_{S}, S_{J}$, and $J_{S}$ phase transition points are not used for estimating the location of the SV. The reason for this is that these phase transition points $F_{S}, S_{J}$ and $J_{S}$ are dependent on the temporal and spatial development of the congested traffic (Fig. 37). On the other hand, as explained above, the location of $S_{\mathrm{F}}$ phase transition points (dark green triangles in Fig. 37) is of crucial importance for the estimation of the location of the SV. As can be seen, the emergence of the GP stabilizes the fluctuations in the locations of $\mathrm{S}_{\mathrm{F}}$ phase transition points in comparison with the real position of the SV (Fig. 37). Therefore, we can determine that the mean value of the reconstructed location of the SV hardly depends on the time (Fig. 38) after the GP has emerged. In Fig. 38, the mean location of the SV $\bar{x}_{\text {rec }}(t)$ is calculated by formula (9).

Since with lower penetration rate of FCD vehicle $\gamma$ only a few $\mathrm{S}_{\mathrm{F}}$ phase transition points can be registered, it follows that, the smaller the FCD rate $\gamma$, the larger the fluctuations at the reconstructed location of the SV (blue dots in Fig. 38). In contrast, the mean value of the reconstructed location of the $\mathrm{SV}$ is only slightly different when the FCD rate $\gamma$ changes, because the fluctuations at the reconstructed location of the SV have a large effect on the mean value of the reconstructed location of the SV.

We can also see that the mean value of the reconstructed location of the SV increases when the LSP has transformed into the GP (Fig. 38). The reason for this is as follows. The width of the LSP (in the longitudinal direction) and, in particular, the average speed of the vehicles in the LSP change randomly over time. That is why the positions of $\mathrm{S}_{\mathrm{F}}$ phase transition points also fluctuate, because different FCD vehicles accelerate from synchronized flow within the LSP to free flow at different locations. In contrast, the average speed of the vehicles in synchronized flow of the GP stabilizes, because the mean speed in synchronized flow of the GP is strongly influenced by the emergence of wide moving jams [Kerner, 2004].

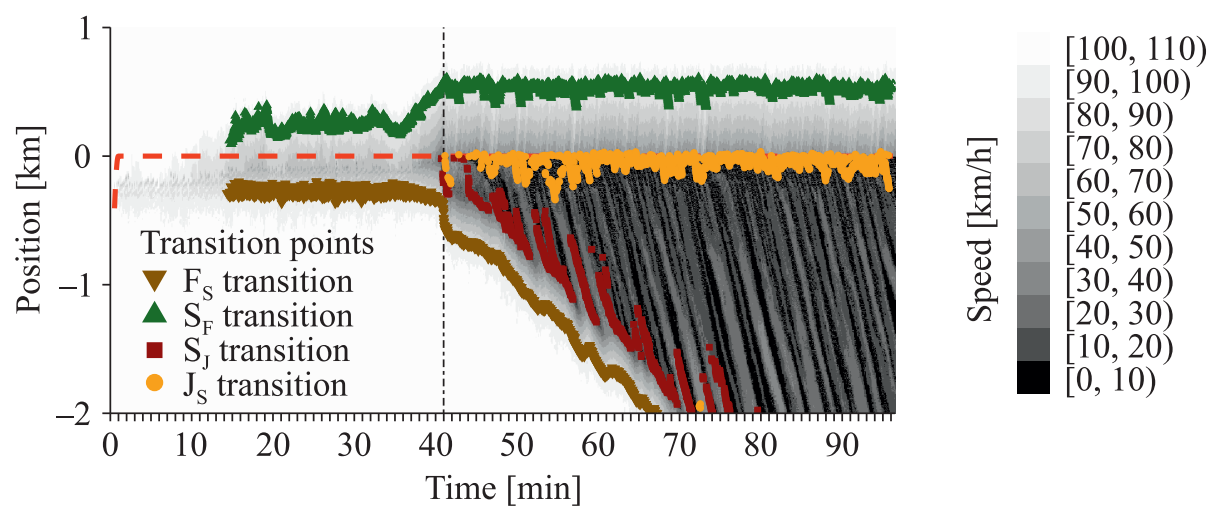

Figure 37. Two-dimensional representation of the simulation results as gray shaded (averaged over both lanes) speed versus road location and time. Phase transition points determined are shown in color: $F_{S}$ as a brown triangle, $\mathrm{S}_{\mathrm{J}}$ as a dark red square, $\mathrm{J}_{\mathrm{S}}$ as a yellow circle, and $\mathrm{S}_{\mathrm{F}}$ as a dark green triangle. The selected time interval corresponds to Figs. 16 and 17 

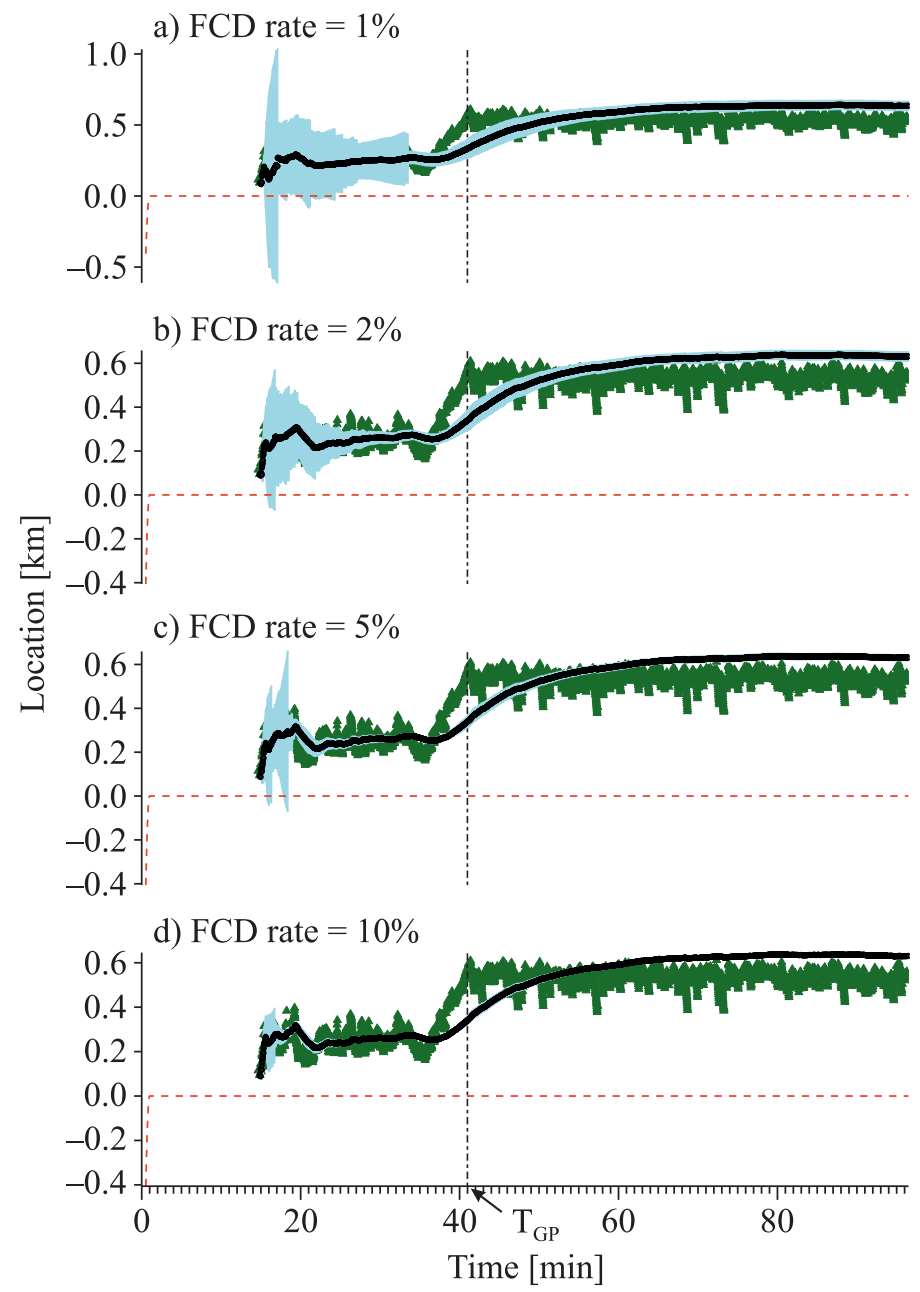

Figure 38. Statistical results for the reconstructed location of the SV from $M=1000$ simulation realizations plotted against the time for 4 different FCD penetration rates. The black dots show the mean value of the reconstructed position of the SV, and the blue area indicates the range of the standard deviation around this mean value and the green triangles show the position of all $\mathrm{S}_{\mathrm{F}}$ phase transition points

\section{Comparison of Prediction of MB and SV}

Simulation examples of the spatiotemporal structures of congested traffic caused by the SV (Fig. 17) and by the MB (Fig. 3) are very different. In particular, there are very different delay times of traffic breakdown $T^{(\mathrm{B})}$ and also the spatiotemporal structures of congested traffic resulting from traffic breakdown $(\mathrm{F} \rightarrow \mathrm{S}$ transition). These differences have a random (statistical) nature that has been understood in Kerner's three-phase traffic theory [Kerner, 2017].

To compare the prediction quality for SV and $\mathrm{MB}$, we remember that the detection of a bottleneck is only possible, when the FCD vehicles have registered $\mathrm{S}_{\mathrm{F}}$ phase transition points. The critical time when the SV has been registered has been denoted by $t_{\mathrm{SV}}$. The critical time when the MB has been registered has been denoted by $t_{\mathrm{MB}}$. For both the SV and the MB, the values $t_{\mathrm{SV}}$ and $t_{\mathrm{MB}}$ can be very different in the above statistical analysis made with the use of $M=1000$ different simulation realizations, in which FCD vehicles have been differently distributed between other vehicles. There is a minimal critical time denoted by $t_{\mathrm{SV}}^{(\mathrm{min})}$, which is related to the prediction of the SV in one of the 1000 realizations. Accordingly, there is a minimum critical time $t_{\mathrm{MB}}^{(\mathrm{min})}$ that is related to the prediction of the MB in one of the 1000 realizations. 

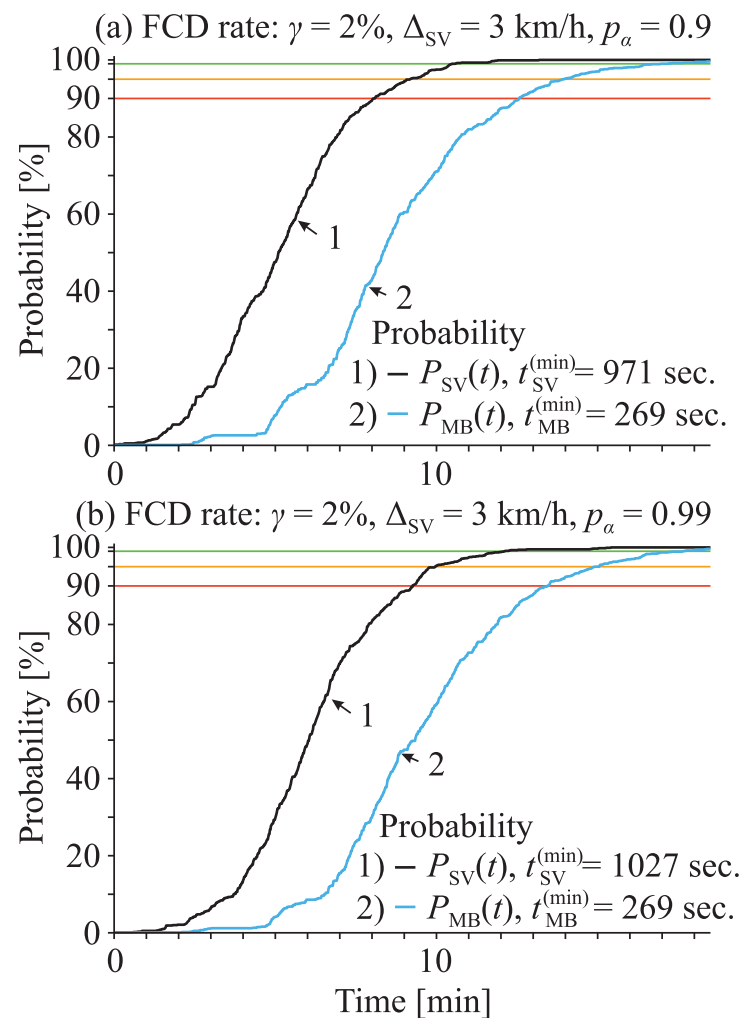

Figure 39. Comparison of the probabilities of the prediction of the SV (curves 1) and the prediction of the MB (curves 2) for the FCD rate $\gamma=2 \%$ (curves 1 and 2) and parameters $\Delta_{\mathrm{SV}}=3 \mathrm{~km} / \mathrm{h}$ (curves 1): (a) Confidence level $p_{\alpha}=0.9$. (b) Confidence level $p_{\alpha}=0.99$. To compare the quality of the prediction of the $\mathrm{SV}$ and the $\mathrm{MB}$, the associated curves of the probabilities have been shifted at the respective critical times $t_{\mathrm{SV}}^{(\min )}$ (curves 1) and $t_{\mathrm{MB}}^{(\mathrm{min})}$ (curves 2), so that at the time $t=0$ at least in one of $M=1000$ realizations either the $\mathrm{SV}\left(t_{\mathrm{SV}}^{(\min )}\right)$ or the $\mathrm{MB}\left(t_{\mathrm{MB}}^{(\min )}\right)$ has been detected, respectively

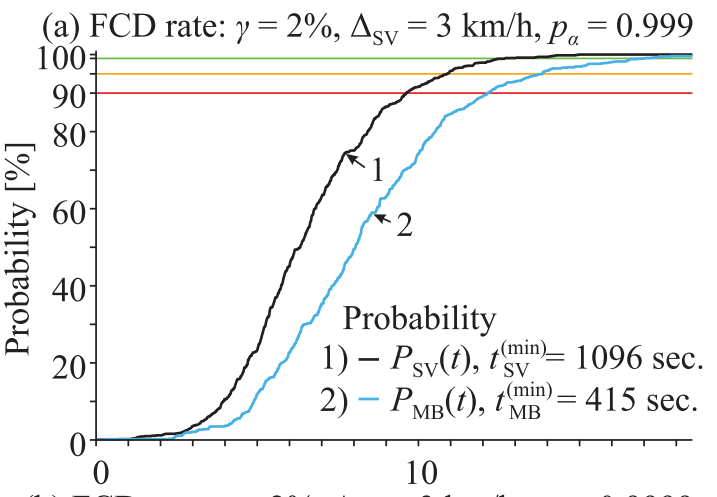

(b) FCD rate: $\gamma=2 \%, \Delta_{\mathrm{SV}}=3 \mathrm{~km} / \mathrm{h}, p_{\alpha}=0.9999$

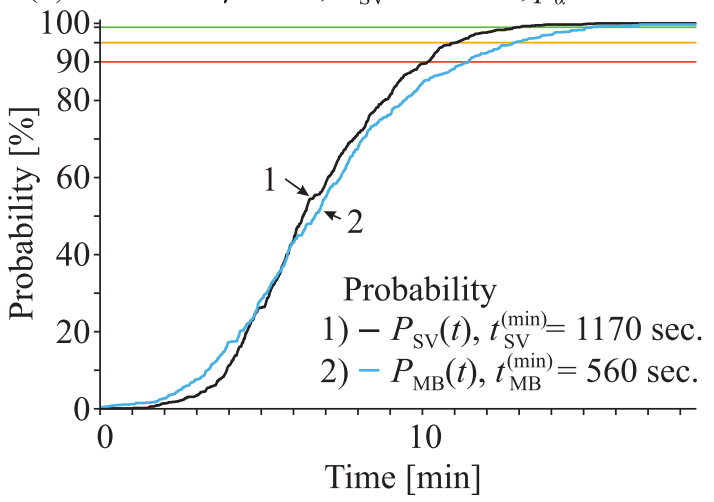

Figure 40. Comparison of the probabilities of the prediction of the SV (curves 1) and the prediction of the MB (curves 2) for the FCD rate $\gamma=2 \%$ (curves 1 and 2) and parameters $\Delta_{\mathrm{SV}}=3 \mathrm{~km} / \mathrm{h}$ (curves 1): (a) Confidence level $p_{\alpha}=0.999$. (b) Confidence level $p_{\alpha}=0.9999$. To compare the quality of the prediction of the SV and the MB, the associated curves of the probabilities have been shifted at the respective critical times $t_{\mathrm{SV}}^{(\min )}$ (curves 1) and $t_{\mathrm{MB}}^{(\mathrm{min})}$ (curves 2), so that at the time $t=0$ at least in one of $M=1000$ realizations either the $\mathrm{SV}\left(t_{\mathrm{SV}}^{(\mathrm{min})}\right)$ or the $\mathrm{MB}\left(t_{\mathrm{MB}}^{(\mathrm{min})}\right)$ has been detected, respectively

To make a statistical comparison of the quality of the prediction of the SV and of the prediction of the MB, we move the curves of the calculated probabilities of the prediction of the SV and the prediction of the MB on the time axis at the respective minimum critical times $t_{\mathrm{SV}}^{(\min )}$ (curves 1) and $t_{\mathrm{MB}}^{(\min )}$ (curves 2) (Figs. 39, 40, 41 and 42). The following results have been obtained:

1. If the values of the confidence level are $p_{\alpha}=0.9$ and 0.99 , there is a big difference in the probabilities of the prediction of the SV and the prediction of the MB: The probability of the prediction of the MB is much smaller than the probability of the prediction of the SV.

The reason for this result lies in the different effects of the MB and the SV on traffic flow. A traffic breakdown at the MB occurs at much larger traffic flow rates than the flow rates at which traffic breakdown occurs at the SV. When the traffic flow rate increases, the probability of the occurrence of sequences of $\mathrm{F} \rightarrow \mathrm{S} \rightarrow \mathrm{F}$ transitions increases significantly. That is why there are some $S_{\mathrm{F}}$ phase transition points occurring at the MB before a traffic breakdown has occurred at the MB. Conversely, the probability of $\mathrm{F} \rightarrow \mathrm{S} \rightarrow \mathrm{F}$ transitions at smaller flow rates at which a traffic breakdown occurs at the SV is small. For this reason, in the example of the SV (Fig. 16) there are no F $\rightarrow \mathrm{S} \rightarrow \mathrm{F}$ 

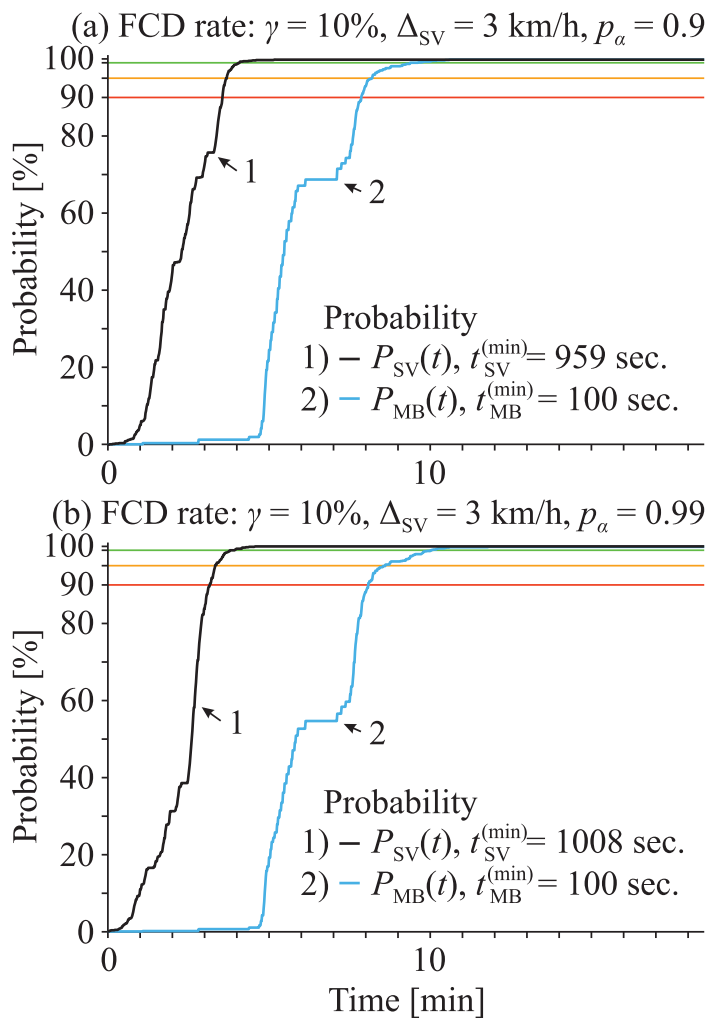

Figure 41. Comparison of the probabilities of the prediction of the SV (curves 1) and the prediction of the MB (curves 2) for the FCD rate $\gamma=10 \%$ (curves 1 and 2) and parameters $\Delta_{\mathrm{SV}}=3 \mathrm{~km} / \mathrm{h}$ (curves 1): (a) Confidence level $p_{\alpha}=0.9$. (b) Confidence level $p_{\alpha}=0.99$. To compare the quality of the prediction of the $\mathrm{SV}$ and the $\mathrm{MB}$, the associated curves of the probabilities have been shifted at the respective critical times $t_{\mathrm{SV}}^{(\mathrm{min})}$ (curves 1) and $t_{\mathrm{MB}}^{(\mathrm{min})}$ (curves 2), so that at the time $t=0$ at least in one of $M=1000$ realizations either the $\mathrm{SV}\left(t_{\mathrm{SV}}^{(\mathrm{min})}\right)$ or the $\mathrm{MB}\left(t_{\mathrm{MB}}^{(\mathrm{min})}\right)$ has been detected, respectively

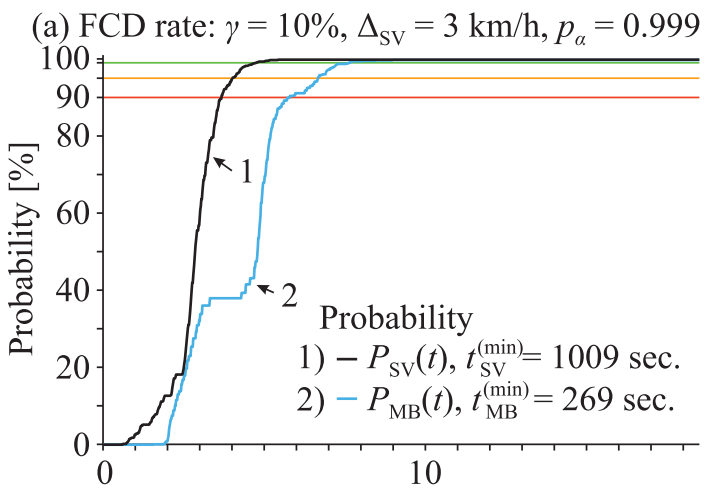

(b) FCD rate: $\gamma=10 \%, \Delta_{\mathrm{SV}}=3 \mathrm{~km} / \mathrm{h}, p_{\alpha}=0.9999$

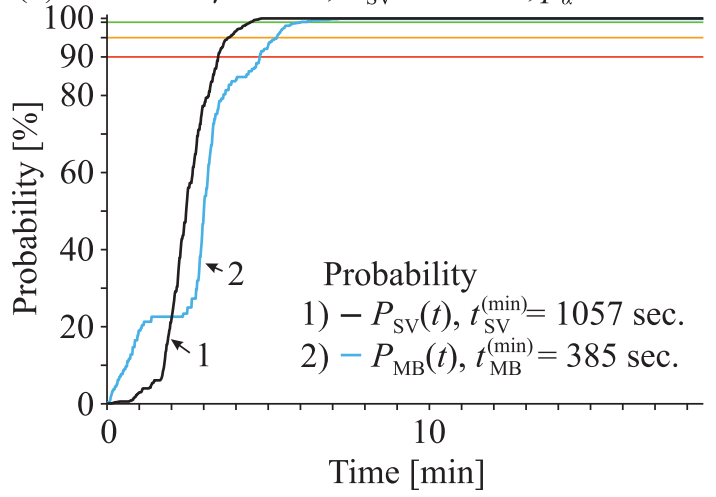

Figure 42. Comparison of the probabilities of detecting the $\mathrm{SV}$ (curves 1) and the MB for the FCD rate $\gamma=10 \%$ (curves 1 and 2) and parameters $\Delta_{\mathrm{SV}}=3 \mathrm{~km} / \mathrm{h} \quad$ (curves 1): (a) Confidence level $p_{\alpha}=0.999$. (b) Confidence level $p_{\alpha}=0.9999$. To compare the quality of the detection of the stopped vehicle (SV) and a moving bottleneck (MB), the curves of the probabilities at the respective critical times $t_{\mathrm{SV}}^{(\mathrm{min})}$ (curves 1) and $t_{\mathrm{MB}}^{(\mathrm{min})}$ (curves 2) have been shifted, so that at the time $t=0$ at least in a realization of $M=1000$ realizations either the $\mathrm{SV}\left(t_{\mathrm{SV}}^{(\mathrm{min})}\right)$ or the $\mathrm{MB}\left(t_{\mathrm{MB}}^{(\min )}\right)$ have been detected

transitions; therefore, there are no $S_{\mathrm{F}}$ phase transition points before a traffic breakdown at SV occurs. This physical result has two consequences:

(i) On the one hand, the $\mathrm{F} \rightarrow \mathrm{S} \rightarrow \mathrm{F}$ transitions at the MB allow us to detect the MB much earlier than the SV bottleneck at which no $\mathrm{F} \rightarrow \mathrm{S} \rightarrow \mathrm{F}$ transitions are realized. That becomes clear when the values $t_{\mathrm{SV}}^{(\mathrm{min})}$ and $t_{\mathrm{MB}}^{(\mathrm{min})}$ in Figs. 39 and 40 are compared with each other.

(ii) On the other hand, the probability of the prediction of the MB increases much more slowly over time in comparison with the probability of the prediction of the SV (Fig. 39). This is the result of the significantly small number of $S_{\mathrm{F}}$ phase transition points associated with $\mathrm{F} \rightarrow \mathrm{S} \rightarrow \mathrm{F}$ transitions that have occurred at the $\mathrm{MB}$ in comparison with a significantly larger number of $S_{\mathrm{F}}$ phase transition points that appear after a traffic breakdown has already occurred. In other words, while the SV (in the example under consideration) cannot be recognized before the traffic breakdown takes place, the SV can be detected very quickly after the traffic breakdown has occurred.

2. The result that the probability of the prediction of the MB grows over time much more slowly than the probability of the prediction of the SV grows over time (Fig. 39) almost disappears after the 
confidence level $p_{\alpha}$ is raised on 0.9999 (Fig. 40). The reason for this is as follows. With such a high level of confidence $p_{\alpha}$ one needs a relatively large number of $S_{\mathrm{F}}$ phase transition points to recognize either the MB or the SV has been detected. Such a number of $S_{\mathrm{F}}$ phase transition points is difficult to get through the occurrence of $\mathrm{F} \rightarrow \mathrm{S} \rightarrow \mathrm{F}$ phase transitions. Therefore, at the confidence level $p_{\alpha}=0.9999$ both the MB and the SV can be detected and distinguished from each other only after a traffic breakdown has already occurred at a bottleneck caused by the MB or the SV. In this case, the "advantage" of the prediction of the MB in comparison with the prediction of the SV caused by the occurrence of $\mathrm{F} \rightarrow \mathrm{S} \rightarrow \mathrm{F}$ transitions at the MB disappears.

These qualitative results persist when the FCD rate increases from $\gamma=2 \%$ (Figs. 39 and 40 ) to $\gamma=10 \%$ (Figs. 41 and 42). However, the "advantage"of the prediction of the MB due to $\mathrm{F} \rightarrow \mathrm{S} \rightarrow \mathrm{F}$ transitions disappears not completely if the confidence level $p_{\alpha}$ is increased to 0.9999 (Fig. 40). The reason for this result is that at the FCD rate $\gamma=10 \%$ the number of $S_{\mathrm{F}}$ phase transition points resulting from $\mathrm{F} \rightarrow \mathrm{S} \rightarrow \mathrm{F}$ transitions increases considerably when the FCD rate $\gamma$ increases. Therefore, the MB can be recognized more easily at larger FCD rates $\gamma$. Additionally, as the FCD rate increases, the

value $t_{\mathrm{MB}}^{(\mathrm{min})}$ decreases. Thus, the probability of the prediction of the MB before a traffic breakdown has occurred at the MB increases considerably (Figs. 39 and 40).

\section{Discussion}

\section{About Possible Applications of the Results of the Paper for Real-Life Situations}

It should be emphasized that the single case illustration of the method of MB and SV prediction given in this paper is related to the finite paper length only. We have simulated many other different scenarios of traffic breakdown at the MB related to different MB speeds and flow rate values. All simulations have led qualitatively to the same conclusions as those found in this paper for the given illustration of the methodology of MB and SV prediction (Figs. 2-14).

As mentioned above, probably due to the absence of real field traffic data of observations of traffic breakdown at a MB (without the influence of road bottlenecks), we know no detailed empirical studies of traffic breakdown at the MB. However, at least in simulations with the KK model used in this paper, the traffic breakdown exhibits the same nucleation nature at the MB as that at a road bottleneck. This fact can be seen from a comparison of Figs. 2 and 3: In a system coordinate moving at the MB speed (Fig. 3), the traffic breakdown exhibits qualitatively the same features as those found at other road bottlenecks (see, e.g., illustrations of empirical traffic breakdowns at road bottlenecks and related simulations in the books [Kerner, 2004; Kerner, 2009; Kerner, 2017]).

The KK model used in this paper has been validated and calibrated based on a number of real field traffic data measured between 1996 and 2018 on many highways in different countries. The model can show and explain spatiotemporal empirical traffic patterns found in the traffic data. Therefore, we can assume that the absence of real data for traffic breakdown at a MB does not restrict our conclusions about the applicability of the methodology of MB prediction presented in this paper for real ITS-applications.

\section{Conclusions}

A general simulation methodology presented in this paper, which is based on Kerner's threephase traffic theory, allows us both to predict the unexpected bottleneck that suddenly occurs on a highway and to distinguish between the origin of the unexpected bottleneck, i.e., whether the unexpected bottleneck has occurred due to an MB or an SV.

1. An MB or an SV can be predicted if a significant vehicle speed disturbance has occurred at the MB or the SV bottleneck, so that there are at least two $S_{\mathrm{F}}$ phase transition points registered through the use of FCD-vehicles. 
2. A methodology of MB and SV prediction can be as follows:

(i) Through the use of FCD-vehicles that are randomly distributed between other vehicles, $\mathrm{S}_{\mathrm{F}}$ phase transition points on FCD-vehicle trajectories are identified. If a $\mathrm{S}_{\mathrm{F}}$ phase transition point is detected, it is assumed that there is a bottleneck on the road.

(ii) Based on two or more $\mathrm{S}_{\mathrm{F}}$ phase transition points, the bottleneck speed and the error in the speed are estimated over time. Taking into account the error, it is proven at each time instant that at a chosen confidence level the bottleneck moves with a positive speed. In this case, the conclusion is drawn that the bottleneck is an MB. Estimations of MB speed and location are sent to automated driving vehicles. The same methodology is used to recognize the SV bottleneck caused by the stopped (broken-down) vehicle.

3. $\mathrm{F} \rightarrow \mathrm{S} \rightarrow \mathrm{F}$ transitions and large speed oscillations in synchronized flow can have a considerable effect on the probability of the MB and the SV prediction and on the accuracy of the estimation of MB and SV location.

In the paper, the simulation examples of the MB and the SV are used to illustrate a general methodology only. We believe that the application of the general methodology for the MB and SV prediction for other types of MBs and SVs that is beyond the scope of this paper could be an interesting topic for further research.

\section{Список литературы (References)}

Bronštejn I. N. Taschenbuch der Mathematik. - Verlag Harri Deutsch, 2001.

Chanut S., Leclercq L. Introducing the Effects of Slow Vehicles in a LWR Two-Flow Traffic Model // Traffic and Granular Flow '03 / Eds. S. P. Hoogendoorn, S. Ludig, P. H. L. Bovy, M. Schreckenberg, D. E. Wolf. - Heidelberg, Berlin: Springer, 2005. - P. 241-246.

Daganzo C. F., Laval J.A. On the numerical treatment of moving bottlenecks (Report UCB-ITS-RR93-7). - Berkeley, CA: Institute of Transportation Studies, 2003.

Daganzo C. F., Laval J. A. On the numerical treatment of moving bottlenecks // Transportation Research Part B: Methodological. - 2005. - Vol. 39, No. 1. - P. 31-46.

Daganzo C.F., Laval J.A. Moving bottlenecks: A numerical method that converges in flows // Transportation Research Part B: Methodological. - 2005. - Vol. 39, No. 9. - P. 855-863.

Draper N. R., Smith H. Applied Regression Analysis. - NY: Wiley, 1998.

Dülgar Y., Molzahn S.-E., Rehborn H., Koller M., Kerner B.S., Wegerle D., Schreckenberg M., Menth M., Klenov S. L. Empirical random phase transitions between free flow and synchronized flow at highway bottlenecks // Journal of Intelligent Transportation Systems. - 2020. - Vol. 24, No. 6. - P. 539-555.

Elefteriadou L. An introduction to traffic flow theory. - New York: Springer, 2014.

Fadhloun K., Rakha H., Loulizi A. Impact of underlying steady-state fundamental diagram on moving bottleneck passing rates using a second-order traffic model // Transportation Letters. - 2014. Vol. 6, No. 4. - P. 185-196.

Fadhloun K., Rakha H., Loulizi A. Comprehensive Framework for Estimating Traffic Stream Flow Rates past Moving Bottlenecks // Transportation Research Record: Journal of the Transportation Research Board. - 2014. - Vol. 2422, No. 1. - P. 61-70.

Fadhloun K., Rakha H., Loulizi A. Analysis of moving bottlenecks considering a triangular fundamental diagram // International Journal of Transportation Science and Technology. - 2016. - Vol. 5, No. 3. - P. 186-199. 
Fadhloun K., Rakha H., Loulizi A. Macroscopic analysis of moving bottlenecks // Transportation Letters. - 2017. - Vol. 36, No. 9. - P. 1-11.

Gazis D. C. Traffic theory. - New York: Springer Science \& Business Media, 2006.

Gazis D. C., Herman R. The Moving and 'Phantom' Bottlenecks. - IBM Thomas J. Watson Research Division, 1990. - $16 \mathrm{p}$.

Gazis D. C., Herman R. The Moving and "Phantom" Bottlenecks // Transportation Science. - 1992. Vol. 26, No. 3. - P. 223-229.

Gorroochurn P. Classic Topics on the History of Modern Mathematical Statistics. - Hoboken, NJ: John Wiley \& Sons, Inc, 2016.

Hausken K., Rehborn H. Game Theoretic Analysis of Congestion, Safety and Security // Springer Series in Reliability Engineering. - Berlin: Springer, 2015. - P. 113-141.

Helbing D. Traffic and related self-driven many-particle systems // Rev. Mod. Phys. - 2001. Vol. 73. - P. 1067-1141.

Hoogendoorn S. P., Bovy P. H. L., Luding S., Schreckenberg M., Wolf D. E. (eds.) Traffic and Granular Flow' 03. - Heidelberg: Springer, 2005. -601 p.

Ke R., Zeng Z., Pu Z., Wang Y. New Framework for Automatic Identification and Quantification of Freeway Bottlenecks Based on Wavelet Analysis // Journal of Transportation Engineering, Part A: Systems. - 2018. - Vol. 144, No. 9. - P. 04018044.

Kerner B. S. A theory of congested traffic flow // Third International Symposium on Highway Capacity, Transportation Research Board Highway Capacity and Quality of Service Committee, Danish Road Directorate. - 1998. - Vol. 2. - P. 621-641.

Kerner B.S. Experimental features of self-organization in traffic flow // Physical review letters. 1998. - Vol. 81, No. 17. - P. 3797-3800.

Kerner B.S. Congested traffic flow: Observations and theory // Transportation Research Record: Journal of the Transportation Research Board. - 1999. - Vol. 1678. - P. 160-167.

Kerner B.S. Theory of congested traffic flow: self-organization without bottlenecks // 14th International Symposium on Transportation and Traffic Theory / A.Cedar (ed.). - Amsterdam: Pergamon, 1999. - P. 147-171.

Kerner B. S. The Physics of Traffic. - Berlin, New York: Springer, 2004. - 682 p.

Kerner B.S. Introduction to modern traffic flow theory and control: the long road to three-phase traffic theory. - Berlin, New York: Springer Science \& Business Media, 2009. - 265 p.

Kerner B.S. Microscopic theory of traffic-flow instability governing traffic breakdown at highway bottlenecks: Growing wave of increase in speed in synchronized flow // Phys. Rev. E. - 2015. Vol. 92. - P. 062827.

Kerner B. S. Breakdown in Traffic Networks: Fundamentals of Transportation Science. - Berlin, New York: Springer, 2017. $-652 \mathrm{p}$.

Kerner B.S. Modeling Approaches to Traffic Breakdown // Complex Dynamics of Traffic Management. Encyclopedia of Complexity and Systems Science Series / B. S. Kerner (ed.). New York, NY: Springer, 2019. - P. 195-284.

Kerner B.S. Spatiotemporal Features of Traffic Congestion // Complex Dynamics of Traffic Management. Encyclopedia of Complexity and Systems Science Series / B. S. Kerner (ed.). New York, NY: Springer, 2019. - P. 387-500.

Kerner B.S. (ed.). Complex dynamics of traffic management. - New York, NY: Springer, 2019.

Kerner B.S., Klenov S.L. A microscopic model for phase transitions in traffic flow // Journal of Physics A: Mathematical and General. - 2002. - Vol. 35, No. 3. - P. L31-L43. 
Kerner B. S., Klenov S. L. Microscopic theory of spatial-temporal congested traffic patterns at highway bottlenecks // Phys. Rev. E. - 2003. - Vol. 60, No. 3. - P. 036130.

Kerner B. S., Klenov S. L. A theory of traffic congestion at moving bottlenecks // Journal of Physics A: Mathematical and General. - 2010. - Vol. 43, No. 42. - P. 425101.

Kerner B. S., Klenov S. L., Hiller A. Criterion for traffic phases in single vehicle data and empirical test of a microscopic three-phase traffic theory // Journal of Physics A: Mathematical and General. 2006. - Vol. 39, No. 9. - P. 2001-2020.

Kerner B.S., Klenov S.L., Hiller A. Empirical test of a microscopic three-phase traffic theory // Nonlinear Dynamics. - 2007. - Vol. 49. - P. 525-553.

Kerner B.S., Rehborn H., Schäfer R.P., Klenov S.L., Palmer J., Lorkowski S., Witte N. Traffic dynamics in empirical probe vehicle data studied with three-phase theory: Spatiotemporal reconstruction of traffic phases and generation of jam warning messages // Physica A: Statistical Mechanics and its Applications. - 2013. - Vol. 392, No. 1. - P. 221-251.

Laval J. A. Stochastic Processes of Moving Bottlenecks // Transportation Research Record: Journal of the Transportation Research Board. - 2006. - Vol. 1988, No. 1. - P. 86-91.

Lebacque J., Lesort J., Giorgi F. Introducing Buses into First-Order Macroscopic Traffic Flow Models // Transportation Research Record: Journal of the Transportation Research Board. 1998. - Vol. 1644. - P. 70-79.

Leclercq L. Bounded acceleration close to fixed and moving bottlenecks // Transportation Research Part B: Methodological. - 2007. - Vol. 41, No. 3. - P. 309-319.

Leclercq L., Chanut S., Lesort J.-B. Moving Bottlenecks in Lighthill-Whitham-Richards Model: A Unified Theory // Transportation Research Record: Journal of the Transportation Research Board. - 2004. - Vol. 1883. - P. 3-13.

Molzahn S.-E., Kerner B.S., Rehborn H., Klenov S.L., Koller M. Analysis of speed disturbances in empirical single vehicle probe data before traffic breakdown // IET Intelligent Transport Systems. - 2017. - Vol. 11, Issue 9. - P. 604-612.

Muñoz J. C., Daganzo C.F. Moving Bottlenecks: A Theory Grounded on Experimental Observation // Transportation and traffic theory in the 21st century / M. A. P. Taylor (ed.). - Amsterdam, New York, Bingley, UK: Pergamon and Emerald Group Publishing Limited, 2002. - P. 441-461.

Newell G.F. A Moving Bottleneck (Technical Report UCB-ITS-RR-93-3). - Berkeley, CA: Institute of Transportation Studies, 1993.

Newell G.F. A moving bottleneck // Transportation Research Part B: Methodological. - 1998. Vol. 32, No. 8. - P. 531- 537.

Rehborn H., Koller M., Kaufmann S. Data-Driven Traffic Engineering: Understanding of Traffic and Applications Based on Three-Phase Traffic Theory. - Amsterdam, Oxford, Cambridge: Elsevier, 2020. $-192 \mathrm{p}$.

Taylor M.A.P. (ed.). Transportation and traffic theory in the 21st century: Proceedings of the 15th International Symposium on Transportation and Traffic Theory, Adelaide, Australia, 16-18 July 2002. - Amsterdam and New York and Bingley, UK: Pergamon and Emerald Group Publishing Limited, 2002. $-439 \mathrm{p}$.

Wegerle D., Kerner B.S., Schreckenberg M., Klenov S. L. Prediction of moving bottleneck through the use of probe vehicles: a simulation approach in the framework of three-phase traffic theory // Journal of Intelligent Transportation Systems: Technology, Planning, and Operations. - 2020. Vol. 24, No. 6. - P. 598-616. 
Yue W. Li C., Mao G. Urban Traffic Bottleneck Identification Based on Congestion Propagation // 2018 IEEE International Conference on Communications (ICC). - 2018. - P. 1-6.

Zhang J., Song G., Yu L., Guo J., Lu H. Identification and characteristics analysis of bottlenecks on urban expressways based on floating car data // Journal of Central South University. - 2018. Vol. 25, No. 8. - P. 2014-2024.

Zheng Z., Ahn S., Chen D., Laval J. Applications of wavelet transform for analysis of freeway traffic: Bottlenecks, transient traffic, and traffic oscillations // Transportation Research Part B: Methodological. - 2011. - Vol. 45, No. 2. - P. 372-384. 
